\title{
A DIFFUSION THEORY MODEL OF SPATIALLY RESOLVED FLUORESCENCE FROM DEPTH DEPENDENT FLUOROPHORE CONCENTRATIONS
}




\title{
A DIFFUSION THEORY MODEL OF SPATIALLY RESOLVED FLUORESCENCE FROM DEPTH DEPENDENT FLUOROPHORE CONCENTRATIONS
}

\author{
By \\ DEREK E. HYDE
}

\begin{abstract}
A Thesis
Submitted to the School of Graduate Studies

in Partial Fulfillment of the Requirements

for the Degree

Master of Science

McMaster University

${ }^{\circ}$ Copyright by Derek E. Hyde, September 1999
\end{abstract}


MASTER OF SCIENCE (1999)

McMaster University

(Medical Physics)

Hamilton, Ontario

TITLE:

A Diffusion Theory Model of Spatially Resolved Fluorescence from Depth Dependent Fluorophore Concentrations

AUTHOR: $\quad$ Derek E. Hyde, B.Sc. (McMaster University)

SUPERVISOR: $\quad$ Dr. T. J. Farrell

NUMBER OF PAGES: viii, 89 


\begin{abstract}
Photodynamic therapy (PDT) currently utilizes drug and light doses which are primarily based on clinical experience. This can lead to a dose which is not sufficient to destroy the entire tumor, or alternatively, it can lead to the undesirable destruction of healthy tissue around the treatment area. PDT of topically applied photosensitizers is one focus of this research. This concerns the diffusion of an externally applied drug into the tissue, as well as its subsequent destruction during the irradiation procedure.

This work involves the non-invasive measurement of the inherent fluorescence of the photosensitizer, allowing the determination of the concentration and distribution of drug within the tissue, and thus optimizing this treatment. To do this, one must be able to describe the propagation of light within the tissue. Consequently, a photon diffusion model has been developed to calculate the steady-state spatially resolved fluorescence from a pencil beam excitation in a depth dependent medium. The validity of this model was then verified by comparison with Monte Carlo simulations and measurements made on phantoms with optical properties similar to those of human tissue. Theoretical conditions were then explored, and potential uses of the model were demonstrated.
\end{abstract}




\section{ACKNOWLEDGEMENTS}

I would like to thank Dr. Tom Farrell for his guidance throughout the course of this work. His insights were often intriguing and his computational abilities were invaluable. I wish to thank Dr. Mike Patterson for his additional direction, as well as his conceptual development. I am grateful to Robert Hawkes for his initial research of this topic, which laid the foundations for the mathematical modeling and phantom development for this project. I am also grateful to Phil Eles for his assistance in writing computer code, and all of the summer and co-op students who have provided assistance in the various aspects of this research.

I wish to thank Dr. Robert Weersink and Jody Bruulsema for their help and advice with the experimental aspects of this research. I would like to thank George Alexandrakis, Kevin Diamond and Rob Hunter for their thought provoking discussions and their assistance within the lab. Finally, I would like to thank everyone at the Robarts Research Institute who provided me with a computer and allowed me to invade their space while I completed this thesis. 


\section{TABLE OF CONTENTS}

Page

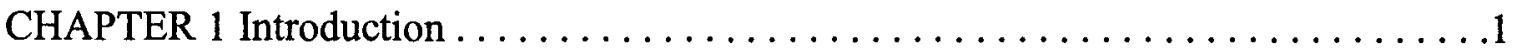

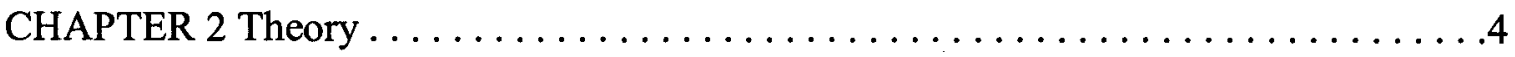

2.1 Photodynamic Therapy $\ldots \ldots \ldots \ldots \ldots \ldots \ldots \ldots \ldots \ldots \ldots \ldots$

2.2 Topical Administration $\ldots \ldots \ldots \ldots \ldots \ldots \ldots \ldots \ldots \ldots \ldots \ldots$

2.3 Light Propagation within a Medium $\ldots \ldots \ldots \ldots \ldots \ldots \ldots \ldots \ldots$

2.4 Bleaching Kinetics $\ldots \ldots \ldots \ldots \ldots \ldots \ldots \ldots \ldots \ldots \ldots \ldots \ldots \ldots$

CHAPTER 3 Mathematical Modeling $\ldots \ldots \ldots \ldots \ldots \ldots \ldots \ldots \ldots \ldots \ldots \ldots \ldots$

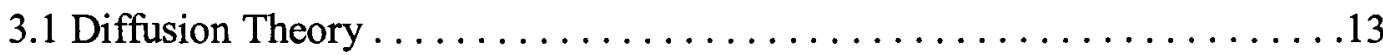

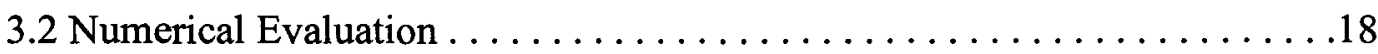

3.3 Monte Carlo Simulation .......................... 26

CHAPTER 4 Experimental Verification. ........................ 30

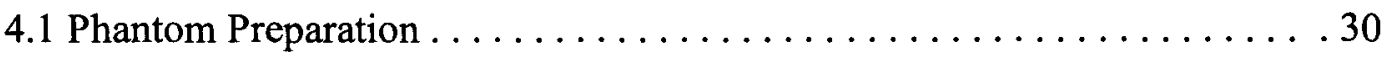

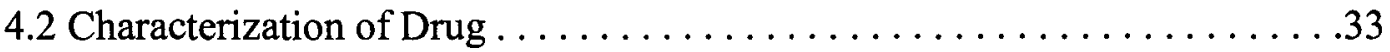

4.3 Layered Phantoms $\ldots \ldots \ldots \ldots \ldots \ldots \ldots \ldots \ldots \ldots \ldots \ldots \ldots \ldots$

4.4 Liquid-Liquid Phantom ............................... 39

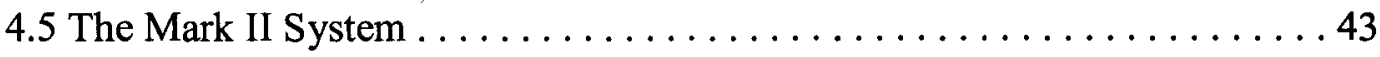

4.6 Measurement Technique. ......................... 45 


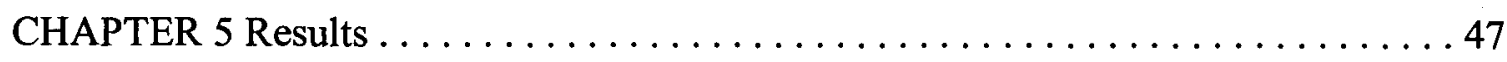

5.1 Drug in Bottom Layer $\ldots \ldots \ldots \ldots \ldots \ldots \ldots \ldots \ldots \ldots \ldots \ldots \ldots \ldots \ldots \ldots \ldots$

Diffusion Theory and Monte Carlo $\ldots \ldots \ldots \ldots \ldots \ldots \ldots \ldots . \ldots 4$

Diffusion Theory and Experiment Measurements ........... 51

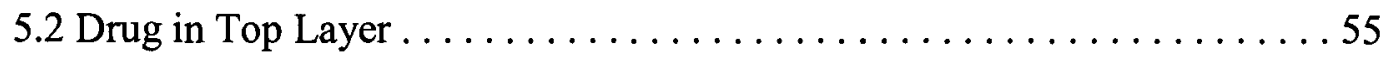

Diffusion Theory and Monte Carlo . . . . . . . . . . . . . .55

Diffusion Theory and Experiment Measurements ........... 59

5.3 Variable Drug Distributions (Theory and Monte Carlo) ............66 66

Simple Exponential Distribution (Similar to Drug Uptake) . . . . . . 66

Sigmoidal Distribution (Similar to Drug Bleaching) .......... 66

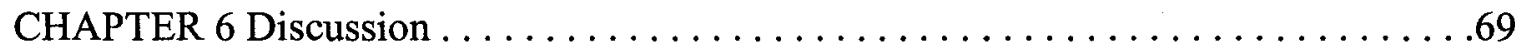

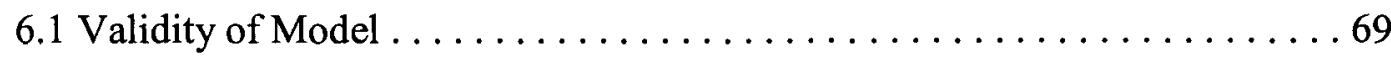

6.2 Modeling the Uptake of a Topically Applied Photosensitizer . . . . . . . . . 70

6.3 Modeling Drug Bleaching During PDT $\ldots \ldots \ldots \ldots \ldots \ldots \ldots \ldots .73$

6.4 Application to a Biological System . . . . . . . . . . . . . . 76

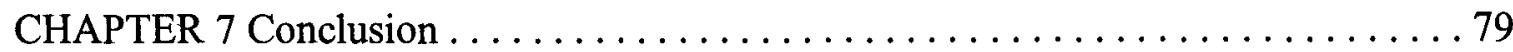

APPENDIX 1: Phantom Materials $\ldots \ldots \ldots \ldots \ldots \ldots \ldots \ldots \ldots \ldots \ldots \ldots \ldots \ldots \ldots \ldots \ldots$

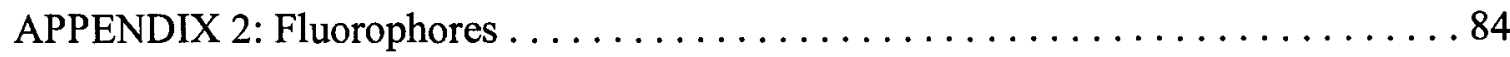

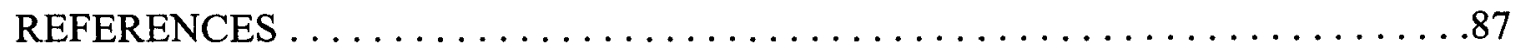




\section{LIST OF TABLES AND FIGURES}

Page

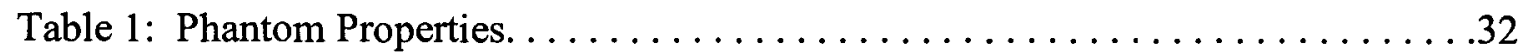

Figure 1: Schematic Energy Diagram of a Photosensitizer. . . . . . . . . . . 5

Figure 2: Fluorescence Fluence $\left\{\phi_{\mathrm{m}}(\mathrm{z}, 0)\right\}$ vs. Depth $\{\mathrm{z}\} \ldots \ldots \ldots \ldots \ldots \ldots \ldots \ldots \ldots \ldots \ldots$

Figure 3a: Comparing Two Step Sizes to Solve $\phi_{x}(z, 0) \& \phi_{m}(z, 0) \ldots \ldots \ldots \ldots .20$

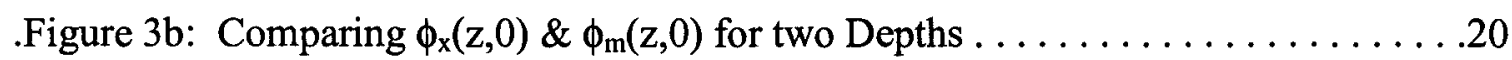

Figure 4: Fluence vs. Spatial Frequency $\ldots \ldots \ldots \ldots \ldots \ldots \ldots \ldots \ldots \ldots \ldots \ldots \ldots$

Figure 5: Determining the number of points for Gaussian Integration $\ldots \ldots \ldots \ldots 23$

Figure 6: Validity of the Integral Approximation (Residual Term) $\ldots \ldots \ldots \ldots 25$

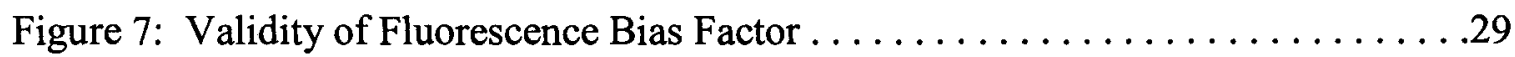

Figure 8: $\mathrm{AlS}_{4} \mathrm{Pc}$ Absorbance vs Concentration .....................

Figure 9: $\mathrm{AlS}_{4} \mathrm{Pc}$ Absorption and Fluorescence $\ldots \ldots \ldots \ldots \ldots \ldots \ldots \ldots \ldots \ldots \ldots$

Figure 10: Schematic for the Detection of Spatially Resolved Fluorescence . . . . . . 37

Figure 11: Two Layer Liquid Phantom $\ldots \ldots \ldots \ldots \ldots \ldots \ldots \ldots \ldots \ldots \ldots \ldots$

Figure 12a: Two Layer Liquid Phantom Reflectance $\ldots \ldots \ldots \ldots \ldots \ldots \ldots \ldots$

Figure 12b: Reflectance Difference for Various Layer Thicknesses . . . . . . . . . . 41

Figure 13a: Two Layer Liquid Phantom Fluorescence ............... 42 
Figure 13b: Fluorescence Difference for Various Layer Thicknesses . . . . . . . . .42

Figure 14: Principle components of the Mark II system $\ldots \ldots \ldots \ldots \ldots \ldots \ldots 44$

Figures 15a-f: Drug in Bottom Layer, Theory and Monte Carlo . . . . . . . . . 48

Figures 16a-f: Drug in Bottom Layer, Theory and Phantom Measurements . . . . . . . 52

Figures 17a-f: Drug in Top Layer, Theory and Monte Carlo ............ 56

Figures 18a-f: Drug in Top Layer, Theory and Phantom Measurements . . . . . . . 60

Figures 19a-f: Drug in Top Layer, Theory and Corrected Measurements . . . . . . 63

Figure 20a: Exponential Drug Concentrations (Similar to Drug Uptake) $\ldots \ldots \ldots \ldots 67$

Figure 20b: Fluorescence from Exponential Concentrations $\ldots \ldots \ldots \ldots \ldots \ldots 6$

Figure 21 a: Sigmoidal Drug Concentration (Similar to Drug Bleaching) $\ldots \ldots \ldots 68$

Figure 21b: Fluorescence from Sigmoidal Concentrations $\ldots \ldots \ldots \ldots \ldots \ldots$

Figure 22a: Drug Concentrations in Normal and Diseased Tissue $\ldots \ldots \ldots \ldots \ldots 71$

Figure 22b: Fluorescence from Normal and Diseased Tissue $\ldots \ldots \ldots \ldots \ldots \ldots 71$

Figure 23a: Drug Concentrations following Topical Application $\ldots \ldots \ldots \ldots \ldots 72$

Figure 23b: Fluorescence following Topical Application $\ldots \ldots \ldots \ldots \ldots \ldots \ldots 72$

Figure 24a: Bleaching of Homogeneous Concentration $\ldots \ldots \ldots \ldots \ldots \ldots \ldots 74$

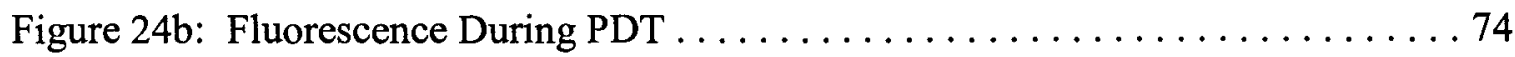

Figure 25a: Drug Concentrations during PDT of Topical Photosensitizer ........ 75

Figure 25b: Fluorescence during PDT of Topical Photosensitizer $\ldots \ldots \ldots \ldots \ldots 75$ 


\section{INTRODUCTION}

Photodynamic therapy (PDT) is a relatively new method of treating cancer and many benign conditions. The therapeutic dose of this technique is dependent on the amount of light absorbed by the photosensitizer within the tissue. Consequently, this treatment provides an inherent dual selectivity. The patient is first administered a photosensitive drug which is preferentially retained by the diseased tissue. Light is then used to activate the drug, and by irradiating only the target tissue, the second part of the selective process is achieved.

Photodynamic therapy of topically applied photosensitizers is the primary focus of this research. This concerns the diffusion of an externally applied drug into the tissue, as well as the subsequent thickness of the layer which is destroyed at the surface during the irradiation procedure [Farrell, 1998]. This is an interesting example because the depth of biological damage is often limited by the diffusion of the topically applied photosensitizer and not by the optical penetration depth, as is the case with systemically administered drugs [Svaasand, 1996].

When a photosensitizer is excited by light, it may produce a cytotoxic product, emit fluorescent light, or become altered itself, a process known as photobleaching. The amount of fluorescence emitted and the rate of toxic species production is related to the amount of the active photosensitizer within the tissue. As the drug is bleached from the 
surface and then deeper into the tissue, there is a resulting decrease in the fluorescence and cytotoxic species production. Thus, if the changing fluorescence signal is observed during treatment, it may be possible to make deductions about the drug distribution within the tissue and, ultimately, the extent of the biological damage. This method of implicit dosimetry has previously been described [Wilson, 1997], in addition to the use of diffuse reflectance spectroscopy [Weersink, 1997], which may provide complementary information, but is generally less sensitive.

It would be advantageous to make a number of non-invasive fluorescent measurements both before and during the irradiation procedure, without perturbing the tissue, so that its effectiveness could be optimized. These measurements can be made at the surface by a probe which has a number of detection fibres located at various distances from a single source fibre, which provides a pencil beam excitation. In order to analyse this fluorescence at the surface of the tissue, one must describe the propagation of light within the tissue.

In earlier work [Hawkes, 1997; Farrell, 1998], the bleaching of drug during PDT was modeled using the finite difference method. As the drug bleached with irradiation time, non-uniform drug distributions were created. Rather than determining the actual drug distribution, the fluorescence was analyzed by creating the equivalent virtual fluorescence source that would produce the same fluorescence distribution at the tissue surface. For the highly absorbing regime, the equivalent source was represented as a single point at some depth within the medium, and scatter did not affect the excitation fluence. For the highly scattering regime it was assumed that the fluence was not altered 
by drug absorption, and the equivalent source was modeled as a plane. The depth of these virtual sources depended on the depth distribution of the photosensitizer, and thus increased as the photobleaching proceeded. These models were limited by the fact that these extreme regimes never actually exist at PDT wavelengths in tissue [Farrell, 1998].

To fully describe the excitation and fluorescence, we have developed a photon diffusion model for the irradiation of the medium with a pencil beam excitation. The cylindrical symmetry of this geometry allows the excitation fluence to be simplified to one dimension using an integral transform, such that it is only dependent on depth. Consequently, the variation of the optical properties of the medium and the fluorophore concentration is also restricted to depth. This is a reasonable approximation for the exponential diffusion of a topically administered photosensitizer into tissue, as well as the photobleaching which would be caused by the broad beam irradiation of the surface. Once the distribution of the excitation is determined, the diffuse fluorescence is modeled, and the steady state spatially resolved fluorescence is calculated at the surface.

This model is an approximation which is not valid in certain regimes and must therefore be validated by comparison with Monte Carlo simulations and actual experimental measurements. These measurements were made on various tissue simulating phantoms constructed with known optical properties, drug concentrations, and drug distributions. This was achieved by the addition of ink to a transparent medium (polystyrene, silicone or water) to simulate the absorption properties of tissue. $\mathrm{TiO}_{2}$ or Intralipid $^{\circledR} /$ Travamulsion $^{\circledR}$ was then added to adjust the scattering within these phantoms. Finally, homogeneous drug concentrations were added to the volumes of interest. 


\section{THEORY}

\subsection{Photodynamic Therapy}

Photodynamic therapy begins with the administration of a photosensitive drug to the patient. This drug is preferentially taken up and retained by diseased tissue. The mechanism of this localization is not entirely understood, but it is thought that the tumorous tissue is unable to remove the aggregated drug because it lacks the necessary lymphatics [McCaughan, 1992]. After an appropriate time delay, the majority of the photosensitizer is often removed from the healthy tissue. The desired treatment volume is then irradiated with a light source which activates the ground state photosensitizer. The additional energy from the absorption of a photon raises the photosensitizer to an excited state (See Figure 1: Schematic Energy Diagram). At this time it may simply emit characteristic fluorescent light and return to its ground state, or it may undergo an intersystem crossing, changing to a triplet state. This energy can then be transferred to (I) surrounding molecules, producing highly reactive free radicals, or (II) a ground state triplet oxygen, producing an excited state singlet oxygen. This is responsible for the majority of the biological damage. Photosensitizers are classified as Type 1 or Type 2, as above, depending on the cytotoxic products they produce.

For oxygen-dependent photosensitization, the photodynamic effect is strongly dependent on local tissue oxygenation. This is contingent on the photochemical depletion 


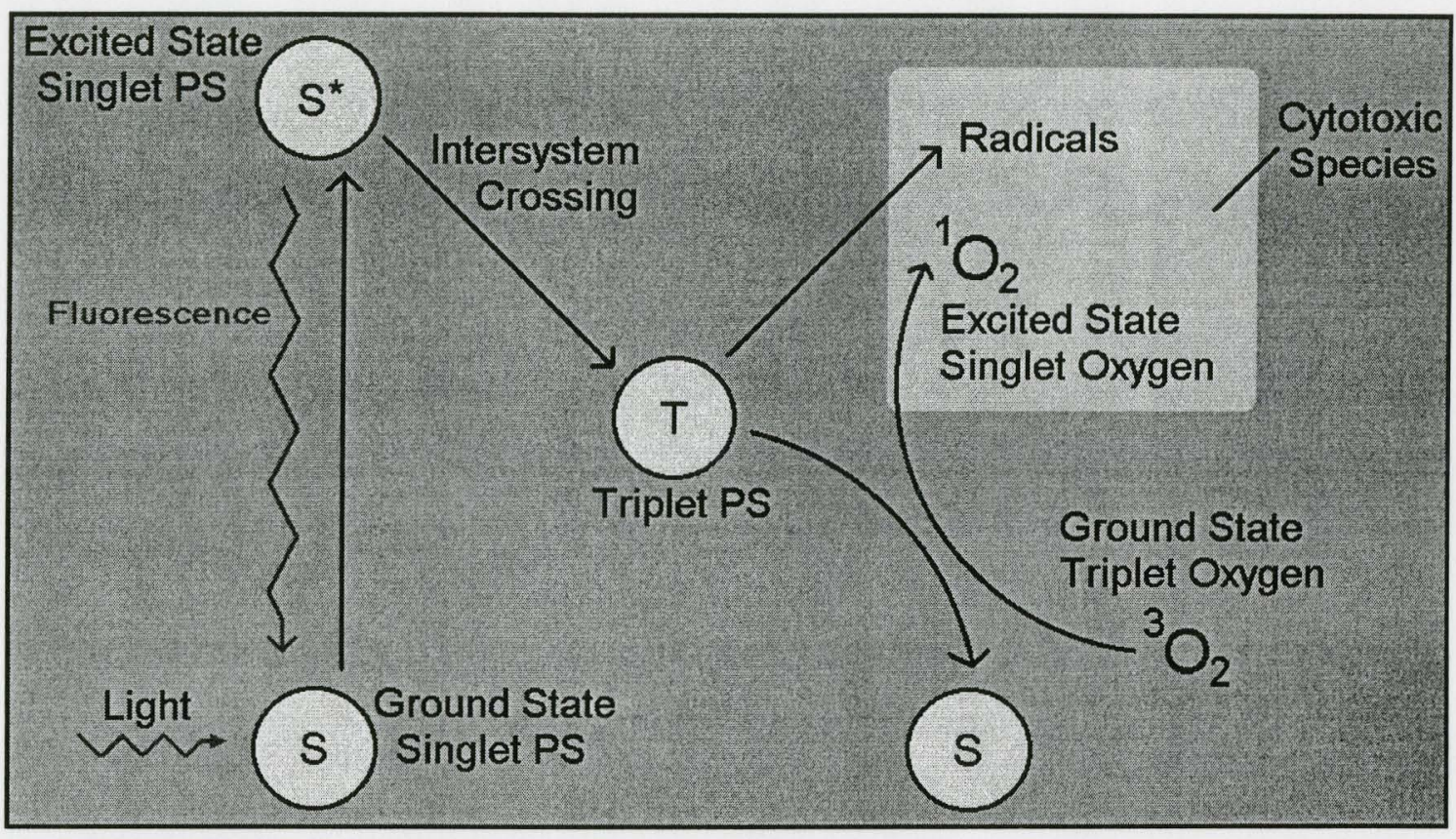

Figure 1: Schematic Energy Diagram of a Photosensitizer (PS). The absorption of a photon raises the ground state singlet photosensitizer to an excited state. At this time it may simply emit characteristic fluorescent light and return to its ground state, or it may undergo an intersystem crossing, changing to a triplet state. Energy can then be transferred to (I) surrounding molecules, producing highly reactive free radicals, or (II) a ground state triplet oxygen, producing an excited state singlet oxygen. This is responsible for the majority of the biological damage 
of oxygen, the perfusion of the tissue, the oxygen diffusion rate from the capillaries, and any vascular changes which occur during the treatment. This is further complicated by the fact that the light penetration is altered by hemoglobin absorption, which is also dependent on the state of oxygenation [Fuchs, 1998].

As the treatment progresses, there is an observed decrease in the measured fluorescence emission, which is known as photobleaching. It should be noted that photobleaching does not necessarily refer to the photochemical destruction of the photosensitizer. It is simply the decreased optical absorbance and/or fluorescence. This corresponds to a loss of photodynamic activity, which is assumed to be related to the photobiological effect on the tissue. Therefore a measurement of the fluorescence may be used to implicitly determine the tissue response, as opposed to individually considering all of the complicated factors which are involved.

A further complication is the fact that if the photosensitizer has a high extinction coefficient at the treatment wavelength, the photosensitizer absorption will be much greater than the intrinsic tissue absorption. This greatly reduces the light penetration, and is known as the self-shielding effect. Consequently, the effective treatment volume is limited by the optical penetration, which continually changes as the photosensitizer is bleached away.

The degree to which the cytotoxic products are responsible for the photobleaching is an important consideration when using implicit dosimetry. If the drug de-activation is mainly caused by photoproducts (i.e. singlet oxygen), the photodynamic effect is said to be 'coupled'. In this case, the photobleaching rate would be proportional to the singlet 
oxygen production, and a high rate would correspond to rapid biological destruction. Conversely, if the de-activation was completely 'uncoupled', rapid photobleaching would indicate a large decrease of the photoactive sensitizer, without the desired biological damage. This extreme situation may be representative of a hypoxic tumour cell, but generally there is some degree of coupling, which complicates the interpretation of the photodynamic effect [Wilson, 1997].

The photochemical damage may result in tissue necrosis by either direct tumor cell death, apoptosis, or by vascular damage leading to ischaemic necrosis. Since the concentration of drug in the healthy tissue is significantly lower than that of the tumor, and the irradiation is tumor specific, the healthy tissue is spared from the treatment. This is further enhanced by the fact that a certain concentration of toxic species is required for necrosis to occur, and the healthy tissue never exceeds this threshold value [Potter, 1986].

\subsection{Topical Administration}

The topical application of a photosensitizer for the treatment of basal cell carcinoma of the skin has been modeled by Svassand et al. The diffusivity, $\kappa$, of the epidermis was estimated from known values for compounds of approximately the same molecular weight $\left(\kappa \sim 10^{-9} \mathrm{~m}^{2} / \mathrm{s}\right)$. It is believed that the increased photosensitizer concentration, in the region of the skin with basal cell carcinoma, is caused by the breakdown of the stratum corneum (protective barrier at surface). Since the diffusivity and time since application were determined, the ratio of the fluorescence from the 
cancerous region to that of the normal adjacent skin was used to determine the permeability of the intact stratum corneum $\left(\mathrm{K}=10^{-7} \mathrm{~m}^{2} / \mathrm{s}\right)$. [Svaasand et al., 1996]

It was assumed that the drug was applied to the surface at $t=0$, and that the surface concentration was kept constant. Using a relaxation time of $\tau=24$ hours tissue, the time dependent drug concentration was determined to be [Svaasand et al., 1996]:

$$
N(t)=N o \int_{0}^{1}\left(\frac{K}{\sqrt{\kappa \pi t^{\prime}}} e^{\frac{x^{2}}{4 \kappa t^{\prime}}}-\frac{K^{2}}{\kappa} e^{\frac{K}{\kappa} \times} e^{\frac{K^{2}}{\kappa} t^{\prime}} \cdot \operatorname{erfc}\left(\frac{K}{\sqrt{\kappa}} \sqrt{t^{\prime}}+\frac{x}{2 \sqrt{\kappa t^{\prime}}}\right)\right) e^{-\frac{t^{\prime}}{\tau}} d t^{\prime}
$$

The steady state depletion of drug across a diffusion barrier (as $t \rightarrow \infty$ ) can be determined by [Svaasand et al., 1996]:

$$
\mathrm{N}(\mathrm{t} \rightarrow \infty)=\frac{\mathrm{No}}{1+\frac{1}{\mathrm{~K}} \sqrt{\frac{\kappa}{\tau}}} \mathrm{e}^{-\frac{\mathrm{x}}{\sqrt{\kappa \tau}}}
$$

The exponential factor represents the steady state decay with distance from the surface, and with these values for healthy tissue, this corresponds to a penetration depth (reduction by $1 / \mathrm{e}$ ) of $9.3 \mathrm{~mm}$. This relatively high value would be typical of a tissue with a high diffusivity and low clearance rate. This is an interesting example from an optics standpoint since the cytotoxic dose delivered to regions close to the site of irradiation is limited by the photobleaching of the drug, rather than by the optical dose. This is simply 
because once the drug is no longer photodynamically active, any additional exposure will not increase the dose. Consequently, this example provides an upper limit for the tissue destruction caused by this treatment, revealing its potential, as well as its harm. It should also be noted that the diffusion barrier is only significant when $\mathrm{K}<<(\kappa / \tau)^{0.5}$, such as with these conditions. [Svaasand et al., 1996]

\subsection{Light Propagation within a Medium}

When studying the propagation of light within a medium, it is usual to assume that all the relevant physics is represented by the steady state photon transport equation [Reichman, 1973; Patterson, 1995] (elastic scattering of monoenergetic photons):

$$
\begin{aligned}
& \mathrm{S}(\mathrm{r}, \Omega)+\left(\mu_{\mathrm{s}} / 4 \pi\right) \int_{4_{\mathrm{r}}} \mathrm{P}\left(\Omega \bullet \Omega^{\prime}\right) \mathcal{R}\left(\mathrm{r}, \Omega^{\prime}\right) \mathrm{d} \Omega^{\prime} \mathrm{drd} \Omega=\Omega \bullet \nabla \mathcal{R}(\mathrm{r}, \Omega)+\mu_{\mathrm{t}} \mathcal{R}(\mathrm{r}, \Omega) \\
& \mathrm{S}(\mathrm{r}, \Omega)= \text { source term }\left(\mathrm{Wsr}^{-1} \mathrm{~m}^{-3}\right) \\
& \mathcal{R}(\mathrm{r}, \Omega)= \text { radiance (angular fluence) at point } \mathrm{r} \text { in direction } \Omega\left(\mathrm{Wsr}^{-1} \mathrm{~m}^{-2}\right) \\
& \mu_{\mathrm{t}}=\text { total interaction coefficient }=\mu_{\mathrm{a}}+\mu_{\mathrm{s}} \\
& \mu_{\mathrm{a}}=\text { absorption coefficient }\left(\mathrm{m}^{-1}\right) \\
&=\text { reciprocal of the mean distance traveled before absorption } \\
& \mu_{\mathrm{s}}=\text { scattering coefficient (m } \left.{ }^{-1}\right) \\
&= \text { reciprocal of the mean-free-path between scattering events } \\
& \mathrm{P}\left(\Omega \bullet \Omega^{\prime}\right)= \text { phase function describing the angular distribution of light } \\
& \text { scattered from direction } \Omega \text { into direction } \Omega^{\prime}
\end{aligned}
$$


If the sources are assumed be isotropic, the general time-independent diffusion equation is derived [Duderstadt, 1976]:

$$
-\mathrm{D} \nabla^{2} \Phi(\mathrm{r})+\mu_{\mathrm{a}} \Phi(\mathrm{r})=\mathrm{S}(\mathrm{r})
$$

where $\mathrm{D}$ is the diffusion coefficient which incorporates a correction for anisotropic scattering:

$$
\begin{aligned}
\mathrm{D} & =1 / 3\left[\mu_{\mathrm{a}}+\mu_{\mathrm{s}}^{\prime}\right] \\
\mu_{\mathrm{s}}^{\prime} & =\mu_{\mathrm{s}}(1-\mathrm{g}) \\
& =\text { reduced scattering coefficient }\left(\mathrm{m}^{-1}\right) \\
\mathrm{g} & =\text { anisotropy parameter } \\
& =\text { mean cosine of the scattering angle }
\end{aligned}
$$

It has been found that if there is a large number of scattering events before detection, different combinations of the scattering coefficient and the phase function lead to indistinguishable light distributions. The scattering properties of two materials are considered to be equivalent when the reduced scattering coefficients are equal [Hulst, 1980]. 
This simplification is generally valid for soft tissues in the range of $600-1300 \mathrm{~nm}$ where $\mu_{\mathrm{a}}<0.1 \mu_{\mathrm{s}}^{\prime}$ [Yoo, 1990]. It may not be valid for heavily pigmented tissues or those with high concentrations of exogenous dyes. Also, the radiance will not be isotropic:

(i) near boundaries such as the interface between tissue and air, where there is an absence of backscattering from the air into the tissue.

(ii) close to highly anisotropic sources, and therefore distances less than a few transport mean free paths $\left(1 / \mu_{s}^{\prime}\right)$ may not be appropriate [Patterson, 1995].

If any of the above conditions are violated, it may be necessary to evaluate the accuracy of the diffusion theory by comparison with Monte Carlo simulations. This involves the analysis of single photons propagating throughout the medium while taking into account the probability of each possible interaction. This becomes a very computationally expensive process in order to produce reasonable counting statistics. Consequently, with the current state of computer technology, it is not economically feasible for 'real-time' analysis, and it is only used for verification.

\subsection{Bleaching Kinetics}

As photobleaching decreases the amount of photosensitizer in the tissue, the light fluence distribution is altered as the total absorption coefficient of the tissue decreases. In a biological environment where this process is strongly coupled to oxygen, the singlet oxygen would combine with surrounding molecules, thereby depleting the local oxygen 
concentration. This progressively reduces the local rate of singlet oxygen production and the local rate of photobleaching (this second order effect was ignored in our model). For simplification, it was assumed that the photosensitizer bleaching rate was only dependent on the fluence rate and photosensitizer concentration. Under these first order conditions, the rate of change of the photosensitizer absorption coefficient can be written as

$$
\frac{d \mu_{a, d}(z, t)}{d t}=-\beta \phi(z, t) \mu_{a, d}(z, t)
$$

where $\beta$ is the photosensitizer bleaching rate. The depth dependent photosensitizer concentration can then be found numerically, using the following approach. The initial fluence rate is calculated according to the distribution of the photosensitizer. The local photobleaching rate is then calculated from the local fluence. The drug absorption coefficient at a time, $\Delta \mathrm{t}$ later, is calculated with

$$
\mu_{a, d}(z, t+\Delta t)=\mu_{a, d}(z, t)-\beta \Phi(z, t) \mu_{a, d}(z, t) \Delta t
$$

The fluence is then solved for the new photosensitizer distribution, and the process is reiterated to calculate the depth dependent drug distribution and fluence rate as the treatment progresses [Farrell, 1998]. 


\section{MATHEMATICAL MODELING}

\subsection{Diffusion Theory}

Photon migration in a two layered medium has previously been modeled by Nossal et al [Nossal, 1988]. This involved the calculation of the exact probabilities for a photons random walk on a discrete lattice. To simplify computations, the concentric symmetry was utilized and the migration space was divided into rings. The migration of the photon was then described by its movement from one annulus to another, its clockwise or counterclockwise movement around the ring, and its lateral position (depth in medium). The fraction which reached the surface was then used to determine the emission profile of the reflectance. This worked well for layers with similar scattering cross-sections but different average absorptions.

A similar two layer model based on the diffusion approximation has also been developed [Kienle, 1998]. To determine the reflectance, the scatter source was simplified as a single point at a depth of one mean free path. He used an extrapolated boundary to account for the mismatch in the index of refraction at the tissue surface. In addition, he used the two dimensional Fourier transform to reduce the three dimensional diffusion equation into a more tractable one dimensional diffusion equation. This was solved and the inverse Fourier transform was taken. The accuracy of this model in determining the optical properties of the two layers has been explored further [Alexandrakis, 1998]. 
Here, the model has been revised to include the diffuse fluorescence, a full description of the scatter source, and any depth dependent variation of the optical properties so that it is no longer restricted to two layers.

The solutions of the steady-state diffusion equation were derived for a medium in which the reduced scatter and absorption coefficients $\left(\mu_{\mathrm{s}}^{\prime}\right.$ and $\left.\mu_{\mathrm{a}}\right)$ and the fluorophore concentration (f) varied only with depth (z). This was done for the reflectance at the excitation wavelength, as well as the fluorescence at the emission wavelength. If the fluorescence excitation and emission spectra overlapped, it would also be possible to extend this model so that reabsorption of the fluorescence could produce another characteristic fluorescence emission. The cylindrical symmetry was taken into account, and the $(\mathrm{x}, \mathrm{y})$ coordinates which lie on the surface of the medium were described by concentric annuli with radii given by $\rho=\left(x^{2}+y^{2}\right)^{1 / 2}$. To describe the intensity that one would measure at the surface, the reflected excitation light, $R(\rho)$, and the escaping fluorescent light, $F(\rho)$, were calculated as a function of radial distance from the pencil beam excitation source incident on the surface at $\rho=0$. Consequently, one must solve the set of coupled diffusion equations for the fluence at the excitation and emission wavelengths, $\Phi_{\mathrm{x}}$ and $\Phi_{\mathrm{m}}$ respectively.

$$
\begin{gathered}
D_{x}(z) \nabla^{2} \Phi_{x}(\vec{r})-\mu_{a, x}(z) \Phi_{x}(\vec{r})=-\mu_{s, x}^{\prime}(z) \delta(x, y) \exp \left[-\int \mu_{t, x}^{\prime}(z) d z\right] \\
\mathrm{D}_{\mathrm{m}}(\mathrm{z}) \nabla^{2} \Phi_{\mathrm{m}}(\overrightarrow{\mathrm{r}})-\mu_{\mathrm{a}, \mathrm{m}}(\mathrm{z}) \Phi_{\mathrm{m}}(\overrightarrow{\mathrm{r}})=-\mu_{\mathrm{a}, \mathrm{x}, \mathrm{d}}(\mathrm{z})\left\{\Phi_{\mathrm{x}}(\overrightarrow{\mathrm{r}})+\delta(\mathrm{x}, \mathrm{y}) \exp \left[-\int \mu_{\mathrm{t}, \mathrm{x}}^{\prime}(\mathrm{z}) \mathrm{dz}\right]\right\}
\end{gathered}
$$


In these equations the subscripts $\mathrm{x}$ and $\mathrm{m}$ refer to the excitation and emission wavelengths and the reduced transport coefficient $\mu_{\mathrm{t}}^{\prime}=\mu_{\mathrm{a}}+\mu_{\mathrm{s}}^{\prime} . \mathrm{D}(\mathrm{z})$ is the depth dependent diffusion coefficient $\left(1 / 3 \mu_{t}^{\prime}\right)$ and $\mu_{\mathrm{a}, \mathrm{x}, \mathrm{d}}$ is the absorption coefficient at the excitation wavelength due to the fluorophore. Note that the source term for the excitation fluence (right hand side of equation 8a) is an extended source along the z-axis with a source strength that is approximately exponentially attenuated with depth. Although this is often simplified as a single scatter source at a depth of one mean free path, the full description was retained due to the added complexity of modeling the fluorescence. The source term for the fluorescence (right hand side of equation $8 b$ ) depends upon the excitation fluence, which cannot be collapsed down to an equivalent single source. The ballistic photons were taken into account, in addition to the scattered photons, since they may also be absorbed by the fluorophore.

The diffusion equations were simplified by transforming them into a set of two coupled one-dimensional differential equations with the use of the two-dimensional Fourier transform

$$
f\left(z, s_{1}, s_{2}\right)=\int_{-\infty}^{\infty} \int_{-\infty}^{\infty} F(x, y, z) \exp \left[i\left(s_{1} x+s_{2} y\right)\right] d x d y
$$

This resulted in the following set of coupled differential equations for the excitation and fluorescence fluence (at each spatial frequency) 


$$
\begin{aligned}
& \frac{d^{2} \phi_{x}(z, s)}{d z^{2}}-\alpha_{x}(z, s)^{2} \phi_{x}(z, s)=-\frac{\mu_{s, x}^{\prime}(z)}{D_{x}(z)} \exp \left[-\int \mu_{t, x}^{\prime}(z) d z\right] \\
& \frac{d^{2} \phi_{m}(z, s)}{d z^{2}}-\alpha_{m}(z, s)^{2} \phi_{m}(z, s)=\frac{\mu_{a, x, d}^{\prime}(z)}{D_{x}(z)}\left\{\phi_{x}(z, s)+\exp \left[-\int \mu_{t, x}^{\prime}(z) d z\right]\right\}
\end{aligned}
$$

where $\alpha(\mathrm{z}, \mathrm{s})^{2}=\mathrm{s}^{2}+\mu_{\mathrm{a}}(\mathrm{z}) / \mathrm{D}(\mathrm{z})$. The photons which are internally reflected are taken into account using the surface boundary condition [Haskell, 1994]:

$$
\phi(\mathrm{z}, \mathrm{s})=2 \mathrm{AD} \frac{\mathrm{d} \phi(\mathrm{z}, \mathrm{s})}{\mathrm{dz}}
$$

where $A=\left(1+R_{\text {eff }}\right) /\left(1-R_{\text {eff }}\right)$ and $R_{\text {eff }}$ is the reflection coefficient which represents the fraction of the emittance that is reflected and becomes irradiance. The black probe at the surface is considered a purely absorbing medium, and therefore $R_{e f f}=0$ and $A=1$. In principle, the one-dimensional diffusion equation (equations $10 \mathrm{a}$ and 10b) can be solved for an arbitrary set of tissue optical properties and fluorophore distribution.

The excitation and fluorescence fluence, $\phi_{x}(0, s)$ and $\phi_{m}(0, s)$, were numerically calculated at each spatial frequency using the method of finite differences [Gerald, 1994]. The two-dimensional Fourier inversions of $\phi_{\mathrm{x}}(0, \mathrm{~s})$ and $\phi_{\mathrm{m}}(0, \mathrm{~s})$ were then evaluated to determine $\Phi_{\mathrm{x}}(0, \rho)$ and $\Phi_{\mathrm{m}}(0, \rho)$. Using the excitation as an example,

$$
\Phi_{x}(0, \rho)=\frac{1}{2 \pi^{2}} \int_{\infty}^{\infty} \int_{\infty}^{\infty} \phi_{x}(0, s) e^{-i\left(s_{1} x+s_{2} y\right)} d s_{1} d s_{2}
$$


and thus,

$$
\Phi_{x}(0, \rho)=\frac{1}{2 \pi} \int_{0}^{\infty} \phi_{x}(0, s) s J_{0}(s \rho) d s
$$

At the surface the reflectance may be expressed as the integral of the radiance over the backward hemisphere. This is expressed as the sum of two terms, one proportional to the fluence rate and one proportional to the current density. Therefore, the steady state reflectance can be written as [Haskell, 1994]

$$
R(0, \rho)=\frac{1}{4 \pi} \int_{2 \pi} d \Omega[1-\operatorname{Rfres}(\theta)]\left[\Phi_{x}(0, \rho)+3 D \frac{\delta \Phi_{x}(0, \rho)}{\delta z} \cos \theta\right] \cos \theta
$$

where $R_{\text {fres }}(\theta)$ is the Fresnel reflection coefficient for a photon with an incident angle $\theta$ relative to the normal of the boundary [Kienle, 1997]. For the refractive index of $n=1.0$, this yields

$$
\mathrm{R}(0, \rho)=0.25 \Phi_{x}(0, \rho)+0.5 \mathrm{D} \frac{\delta \Phi_{x}(0, \rho)}{\delta z}
$$

substitution of the boundary condition (eq. 11), with $A=1$, produces $R(0, \rho)=0.5 \Phi_{x}(0, \rho)$. The spatially resolved fluorescence was then calculated across the surface using $F(0, \rho)=0.5 \Phi_{m}(0, \rho)$ 


\subsection{Numerical Evaluation}

The excitation and fluorescence fluence was calculated using the method of finite differences. Difference equations (eq. 10a) were formed to describe the excitation fluence at each spatial frequency, $\mathrm{R}(\mathrm{s})$, in step sizes of $0.1 \mathrm{~mm}^{-1}$ to a maximum of 55 $\mathrm{mm}^{-1}$. The absorption coefficient and distribution of the drug was used to determine the total absorption and total interaction coefficients $(0.05 \mathrm{~mm}$ step size, $30 \mathrm{~mm}$ maximum depth). The resulting tri-diagonal matrix was solved, yielding $\phi_{x}(z, s)$. These values were then used (in eq 10b) to set up the matrix for the fluorescence fluence, $\phi_{\mathrm{m}}(\mathrm{z}, \mathrm{s})$, which was also solved in the same way. The depth dependent fluence for a spatial frequency of zero is shown in Figure 2. Figure 3a shows that the step size for the depth was appropriate, and Figure 3b shows that the maximum depth was appropriate. A sample of the excitation and fluorescence at the surface is shown in Figure 4.

The two dimensional Fourier inversions were performed for each surface fluence, $\phi_{\mathrm{x}}(0, \mathrm{~s})$ and $\phi_{\mathrm{m}}(0, \mathrm{~s})$, using equation 13. Since the integrands oscillate with the radial distance, the semi-infinite integrals were solved using piece-wise integration between zeros of the Bessel function. The first twenty zeroes of the $0^{\text {th }}$ order Bessel function were tabulated [Abramowitz, p409, 1972] and the first five terms of McMahon's Expansion [Abramowitz, p371, 1972] were used to determine the larger zeros. Spline interpolation was then used to perform Gaussian integration between the zeroes of the Bessel functions. The number of abscissas and weight factors [Abramowitz, p916, 1972] used in 


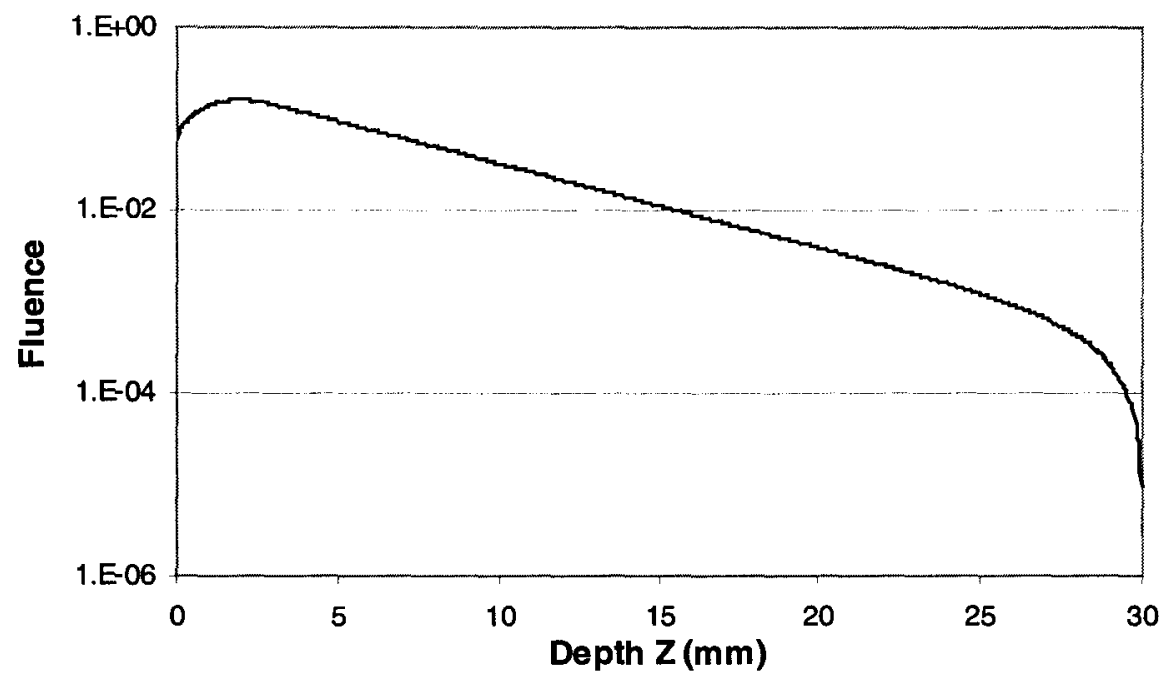

Figure 2: The fluence for a spatial frequency of zero has been logarithmically plotted for a number of depths i.e. $\phi_{\mathrm{m}}(\mathrm{z}, 0)$. The diffusion theory model was used to generate the fluorescence fluence from a $3 \mathrm{~mm}$ top layer with an $\mathrm{AlS}_{4} \mathrm{Pc}_{\mathrm{c}}$ concentration of $2 \mu \mathrm{g} / \mathrm{ml}$, and a $30 \mathrm{~cm}$ tissue equivalent bottom layer (optical properties of $\mu_{\mathrm{s}^{\prime}(@ 632 \mathrm{~nm})}=1.44 \mathrm{~mm}^{-1}$, $\mu_{\mathrm{s}^{\prime}(@ 683 \mathrm{~nm})}=1.14 \mathrm{~mm}^{-1}$, and $\mu_{\mathrm{a}}=0.013 \mathrm{~mm}^{-1}$ for both wavelengths). 


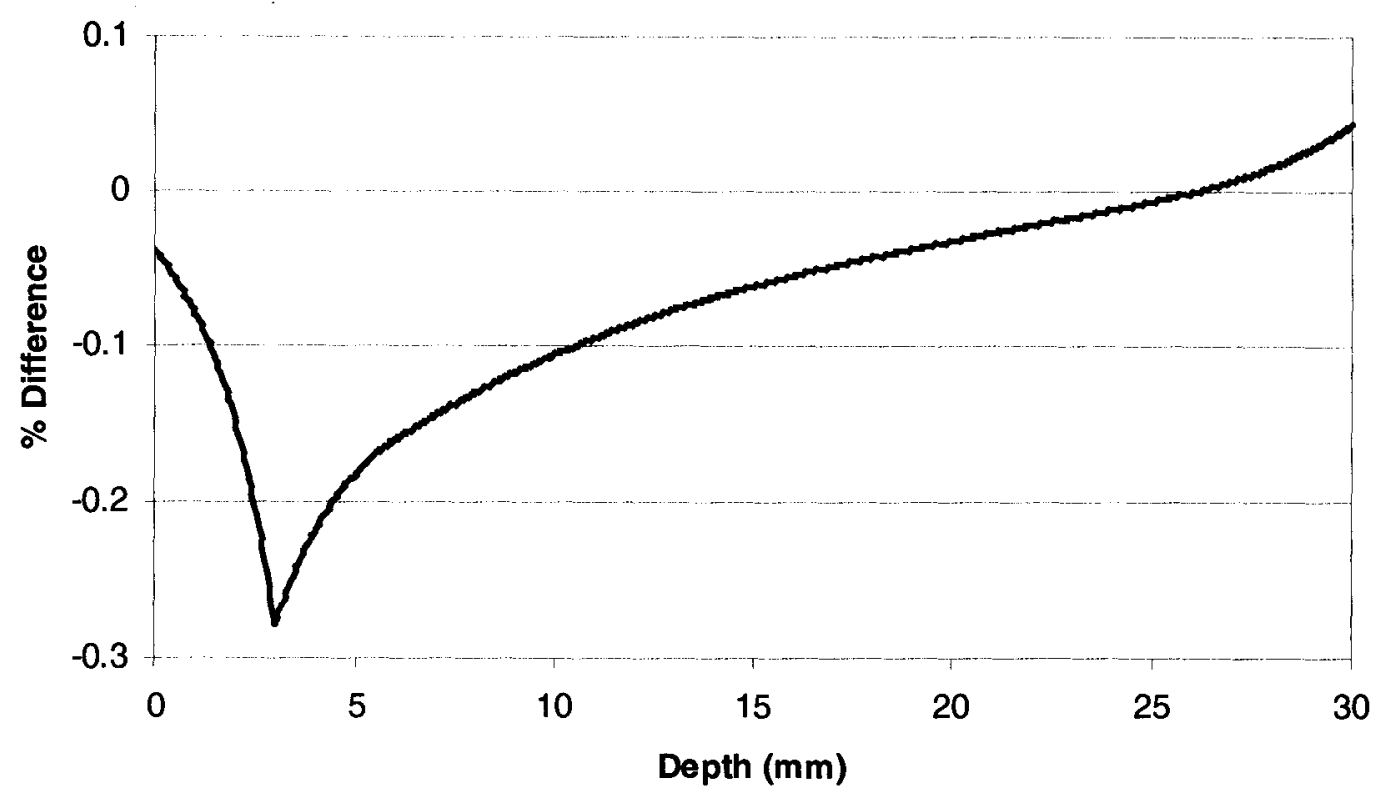

Figure 3a: To determine if the step size was appropriate for the depth $(0.05 \mathrm{~mm})$, the step size was halved, and the percentage difference was plotted at each depth (same conditions as figure 2). Consequently, the step size of $0.05 \mathrm{~mm}$ was considered adequate.

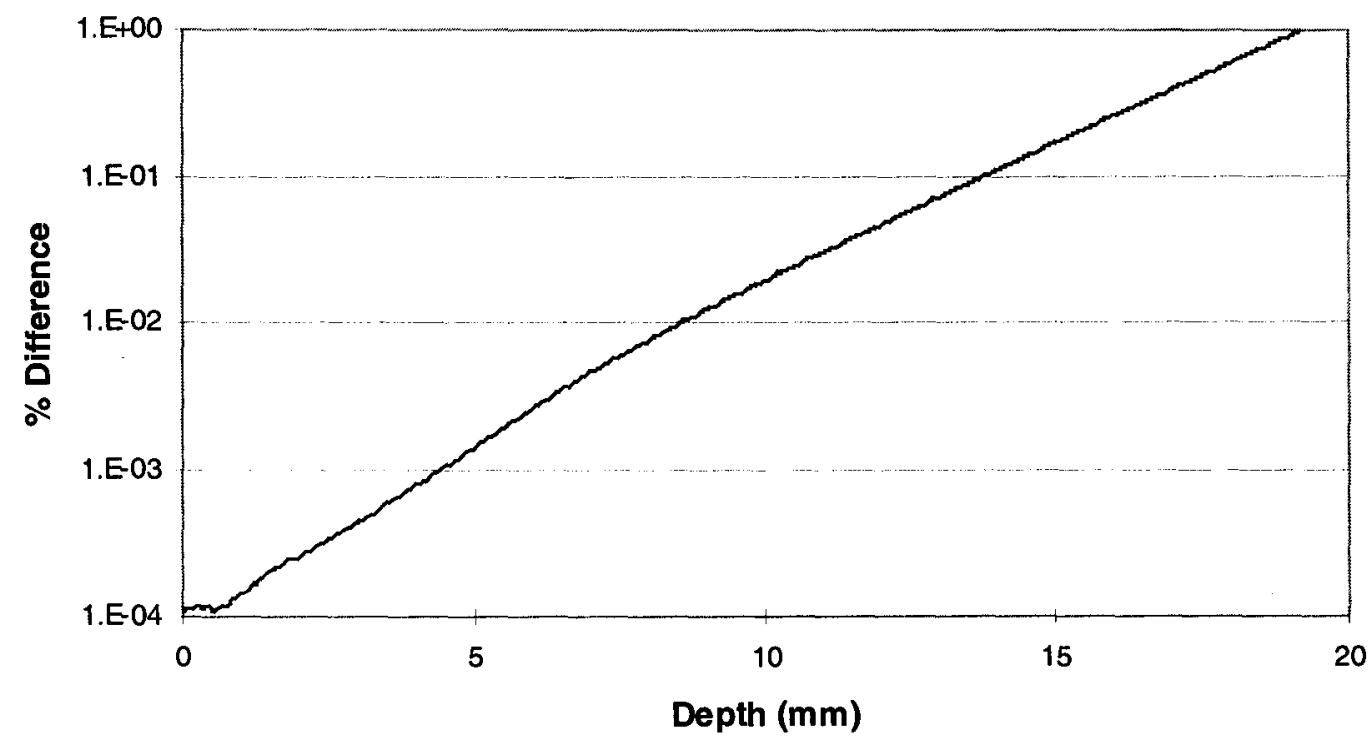

Figure 3b: To determine if the maximum depth was appropriate $(30 \mathrm{~mm})$, it was doubled, and the percentage difference was plotted for depths near the surface (same conditions as above). Consequently, a maximum depth of $30 \mathrm{~mm}$ was considered adequate. 


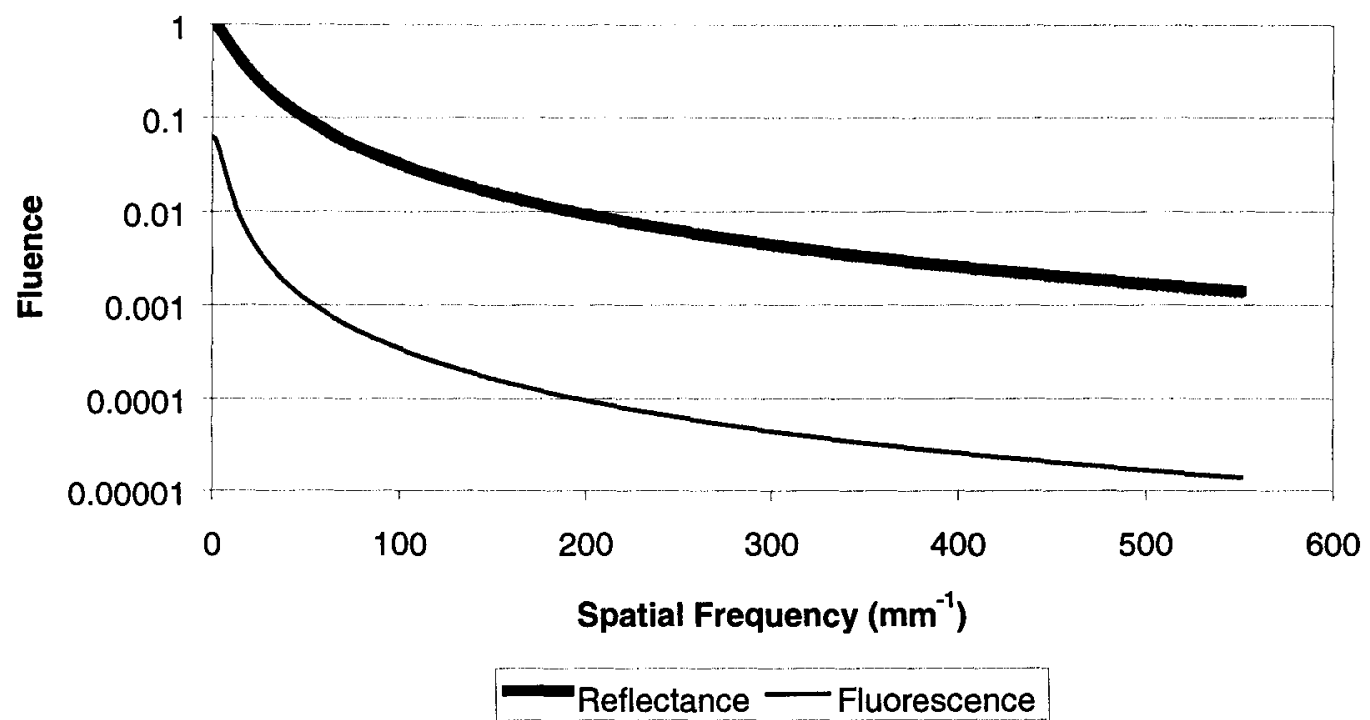

Figure 4: The excitation and fluorescence fluence at the surface, $\phi_{\mathrm{x}}(0, \mathrm{~s})$ and $\phi_{\mathrm{m}}(0, \mathrm{~s})$ respectively, were plotted for the most significant spatial frequencies in step sizes of 0.1 $\mathrm{mm}^{-1}$ (again, the same conditions as figures 2,3 \&4). When the step size was halved, the intensities remained the same, indicating that it would be unnecessary to reduce the step size any further. 
the Gaussian integration were increased until no significant difference was observed (see Figure 5). In practice, ten abscissas and ten weight factors were used.

Previously, when the scatter source was simplified as a single point at a depth of one mean free path, the intensities from the higher spatial frequencies decreased quite rapidly; so the integration could be completed with a reasonable number of spatial frequencies $(\sim 50)$ [Alexandrakis, 1998]. When retaining the full description of the source term, higher frequencies must be considered since they don't decrease as rapidly with the source at the surface. It was found that the integration of up to 180 spatial frequencies was often required to converge to the final solution. This was computationally expensive, and therefore the diminishing contribution of the higher frequencies was estimated by a residual term. For large values of $s \rho$, the Bessel function is approximated [Press, 1996] as

$$
\mathrm{J}_{\mathrm{o}}(\mathrm{s} \rho)=\frac{\cos (\mathrm{s} \rho-\pi / 4)}{\sqrt{\mathrm{s} \rho}}
$$

and as $s \rightarrow \infty$ the solutions of equations $10 \mathrm{a}$ and $10 \mathrm{~b}$ approximate that of the one dimensional homogeneous geometry. Therefore the solution of the diffusion equation becomes $\phi_{x}(z, s)=A \cdot \exp (-\alpha z)+B \cdot \exp \left(-\mu_{t} z\right)$, where $B=\mu_{s}^{\prime} /\left[D\left(\alpha^{2}-\mu_{t}^{2}\right)\right]$ and $A=-B\left(\mu_{t}+2 D\right) /(\alpha+2 D)$, and at $z=0$ becomes

$$
\phi_{\mathrm{x}}(0, \mathrm{~s}) \approx \mu_{\mathrm{s}}^{\prime} / \mathrm{Ds}{ }^{2}
$$




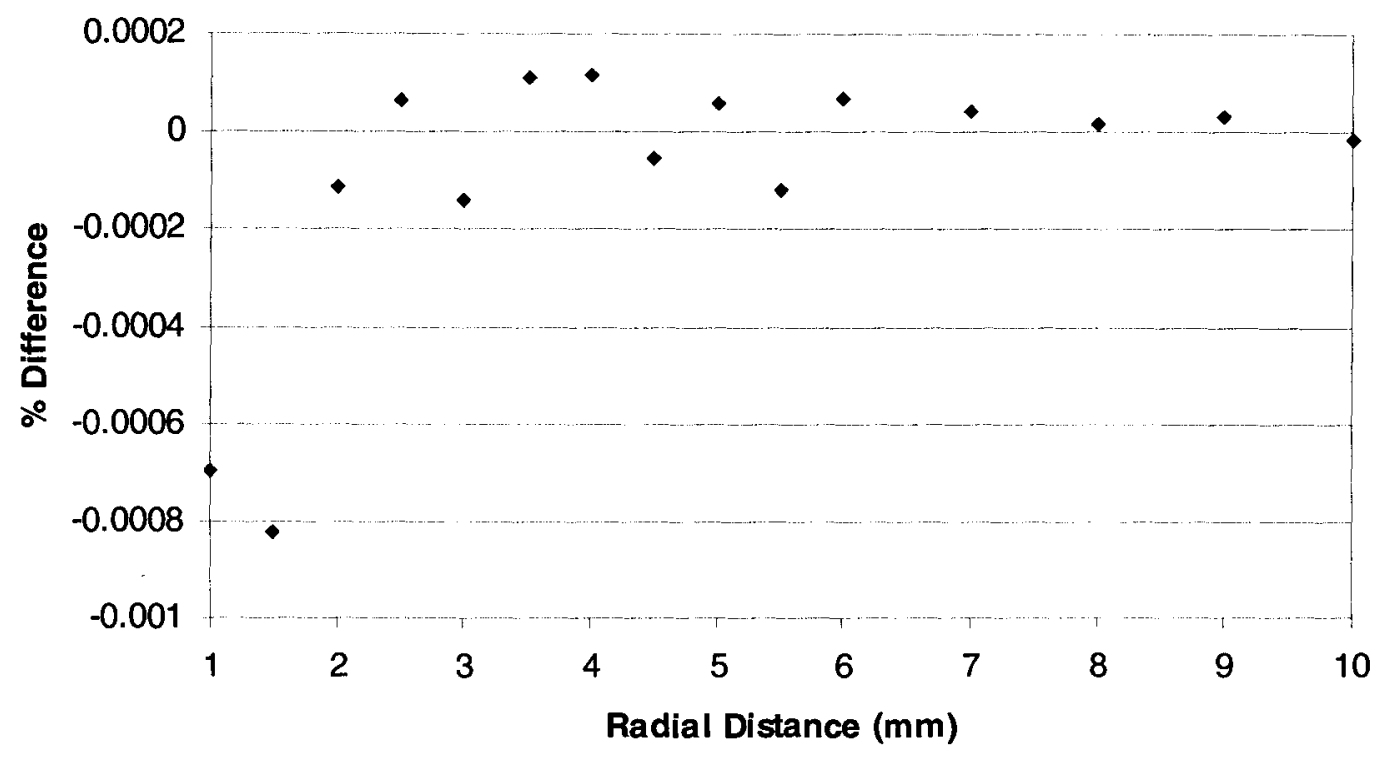

Figure 5: The number of abscissas and weight factors used in the Gaussian integration was doubled (from 20 to 40), and the percentage difference (of the two resulting fluences) was plotted against the radial distance (again the same conditions were used as in the previous figures). 
Substituting these equations (16 and 17) into the two dimensional Fourier inversion (equation 13) produces

$$
\Phi_{\mathrm{x}}(\rho)=\frac{1}{2 \pi} \int_{\mathrm{s}_{0}}^{\infty}\left[\mu \mathrm{s}^{\prime} \cos (\mathrm{s}-\pi / 4)\right] /\left[\mathrm{Ds}^{3 / 2}\right] \mathrm{ds}
$$

which can be solved by repeated integration by parts, and then using the first few terms of the resulting series. Similarly,

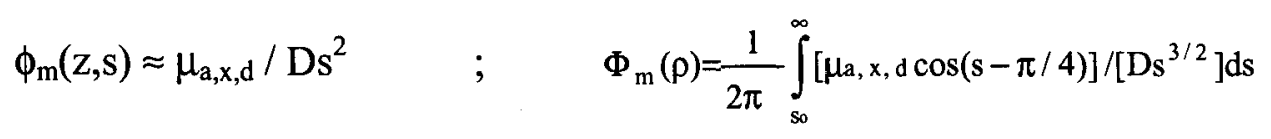

Consequently, the integral of the equation was evaluated out to a significant point, and then the residual contribution was approximated. For a sample of data, a residual value has been added to the integral of the first 55 spatial frequencies for comparison with the calculated data (integrating to 180 spatial frequencies), see Figure 6. This was the standard method of integration for our calculations.

Once the fluence was determined at the surface, the spatially resolved reflectance $R(\rho)$ and escape fluorescence $F(\rho)$ were calculated as described above. 


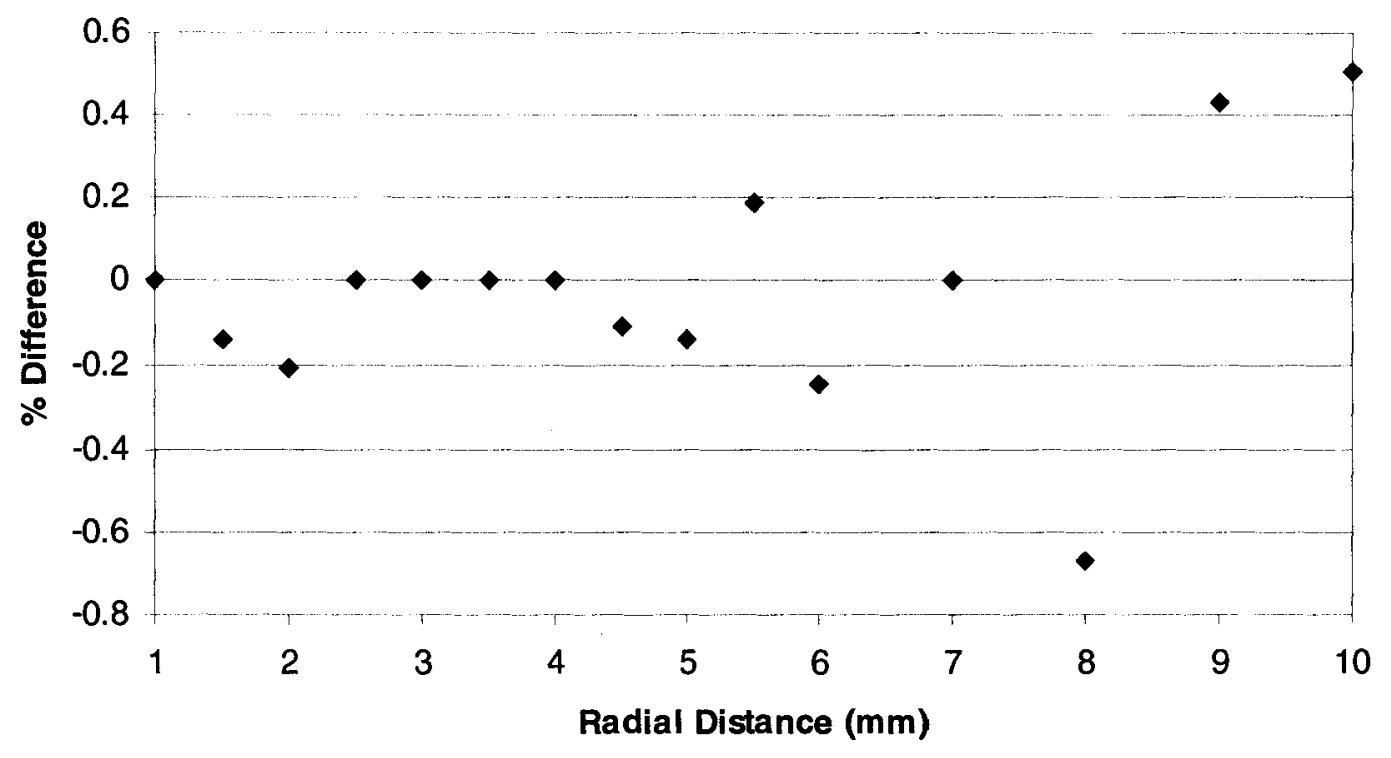

Figure 6: Piece-wise Gaussian integration was used to integrate between the Bessel zeroes until the solution converged after a spatial frequency of $180 \mathrm{~mm}^{-1}$. This was rather computationally expensive, so this integration was only performed up to a spatial frequency of $55 \mathrm{~mm}^{-1}$, and the higher spatial frequencies were approximated by a sinusoidal tail. The percentage difference of the fluorescence fluence was then plotted at a number of radial distances to verify the sinusoidal approximation. 


\subsection{Monte Carlo Simulation}

Monte Carlo techniques, which have been used in a number of radiation transport studies, are based on the stochastic nature of radiation interactions [Profio, 1979]. When studying tissue optics [Hasegawa, 1991], the wave nature of the photons is ignored, and they are considered as neutral particles which propagate in tissue in a manner which is similar to neutrons in a nuclear reactor [Svaasand, 1991]. The only interactions of interest are absorption and elastic scattering, and the medium is described in terms of its absorption and scattering coefficients [Kienle, 1996]. The total absorption is the sum of that of the endogenous chromofluores and any added pigment.

It is assumed that the incident photons enter the medium at $\mathrm{z}=0$, and that the medium occupies the semi-infinite space defined by $z>0$. The photons are launched perpendicular to the medium, and the path length between successive interactions is described by

$$
\mathrm{L}=-\ln (\mathrm{R}) / \mu_{\mathrm{t}}
$$

where $\mathrm{R}$ is a random number between 0 and 1 . This generates an exponential distribution of interaction path lengths. Incidentally, for a medium with a varying drug distribution, the path length is adjusted as a result of the total attenuation coefficient being altered by the drug absorption coefficient $\left(\mu_{\mathrm{t}}=\mu_{\mathrm{s}}+\mu_{\mathrm{a}}+\mu_{\mathrm{a}, \mathrm{d}}\right)$. 
If the medium does not contain any fluorophore, or the fluorophore does not absorb at the wavelength of interest (such as a low energy fluorescence emission), then survival weighting is used. At each interaction point, the photon was assumed to deposit a fraction $\left(\mu_{\mathrm{a}} / \mu_{\mathrm{t}}\right)$ of its current weight, and a new direction of the photon was determined. A new path length was calculated as before, and the photon tracing continued until either its weight was reduced to zero, or it happened to exit back through the surface of the medium. It should be noted that although the photons weight is altered, its energy is not changed by the scatter interaction. Thus, the medium is assumed to have the same absorption and scattering coefficients for both the incident and the scattered photon [Wilson, 1983]. Weighting photons in this manner results in better statistical precision for a given number of input photons, and it is much more computationally efficient than terminating the photons life upon its first absorption [Profio, 1979]. The angular dependence of the scattering is assumed to be independent of the initial direction, and thus a phase function is used to describe the angle between the initial and final paths of the photon. The Henyey-Greenstein function was used as the phase function [Henyey, 1941].

If the photon may be absorbed by a fluorophore within the medium, this absorption must also be taken into account at each interaction point. Therefore the probabiltity of: scattering is $\mu_{s} / \mu_{\mathrm{t}}$, absorption by the medium is $\mu_{\mathrm{a}} / \mu_{\mathrm{t}}$, and absorption by the fluorophore is $\mu_{\mathrm{a}, \mathrm{d}} / \mu_{\mathrm{t}}$. Since drug absorption was relatively low in these experiments, there were relatively few fluorescent events, and therefore the Monte Carlo data were very noisy. To address this problem, a fluorescent bias factor, $\mathrm{B}_{\mathrm{F}}$, was utilized, such that a fraction $\left(\mathrm{B}_{\mathrm{F}}\right)$ 
of the interactions were scatters, and a fraction $\left(1-\mathrm{B}_{\mathrm{F}}\right)$ were fluorescent events. The photon's weight was then adjusted by a factor $\mu_{\mathrm{s}} /\left(\mu_{\mathrm{t}} \mathrm{B}_{\mathrm{F}}\right)$ for the scatter events, and $\mu_{\mathrm{a}, \mathrm{d}} /$ $\left[\mu_{\mathrm{t}}\left(1-\mathrm{B}_{\mathrm{F}}\right)\right]$ for the fluorescence events. This greatly improved the counting statistics; most notably at the shorter radial distances. Optimization of the bias factor was attained by the comparison of Monte Carlo data for a number of values with the theoretical result for a two layer phantom with an aluminum pthalocyanine tetra-sulphonate ( $\mathrm{AlS}_{4} \mathrm{Pc}$ ) concentration of $2 \mu \mathrm{g} / \mathrm{ml}$ in a $3 \mathrm{~mm}$ top layer. As shown in Figure 7, a fluorescent bias factor of 0.995 improved the counting statistics while retaining the shape of the spatially resolved fluorescence curve (although it produced a slightly higher intensity).

In these simulations, a pencil beam was normally incident upon a tissue-equivalent medium. The anisotropy factor $\mathrm{g}$ was chosen to be 0.9 , and the reflectance and fluorescence were scored for distances up to $25 \mathrm{~mm}$ from the source (in annular rings along the surface) with a spatial resolution of $0.02 \mathrm{~mm}$. The data were normalized to the interaction probability for a single photon, and subsequently compared to the diffusion model on an absolute scale. 


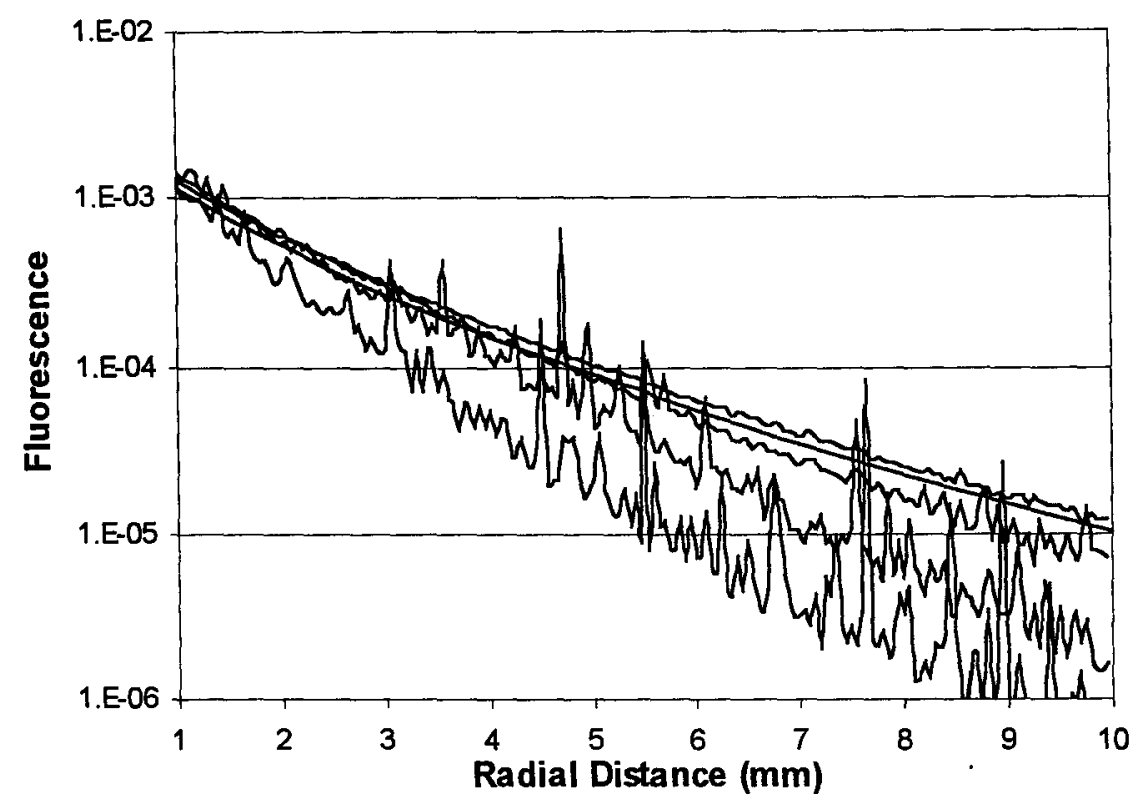

Figure 7: The spatially resolved fluorescence fluence was plotted for a number of bias factors used in the Monte Carlo simulation $(0.85,0.95,0.99,0.995$ from lowest to highest) and compared to the Diffusion Theory (smooth line which has the second highest intensity). The fluorescence bias factor of 0.995 produced the curve which was most similar to the diffusion theory, although its intensity was slightly exaggerated. 


\section{EXPERIMENTAL VERIFICATION}

Phantoms are used to experimentally simulate light propagation in tissue. This allows the experimenter to control the optical properties, drug distribution and drug concentration for comparison with theory. The phantom may be constructed of a number of optically transparent materials, to which an absorber and scatterer are added to adjust the optical properties. Unfortunately the phantom material often affects the properties of the photosensitizer, which complicates the experimental procedure.

\subsection{Phantom Preparation}

As previously noted, the fluorescent properties (yield, lifetime, excitation and emission wavelengths) of the photosensitizers are often altered by the physico-chemical properties of their environment. Consequently, if useful measurements were to be performed, the desired fluorescing material had to be matched with a suitable phantom medium. Ideally a photosensitizer with an absorption wavelength in the near infrared region would be desirable since this is the most transparent region for tissue. Experimentally, the fluorophores absorption coefficient should be prominent at a readily available laser wavelength. In addition, the modeling would be simplified if the drug's absorption at the fluorescent emission wavelength was not significant (only two coupled differential equations would be required for each spatial frequency). In light of these 
intricacies, a number of fluorophores and phantom materials have been analysed. Some of the various phantom properties can be found in the following table. 


\begin{tabular}{|l|c|c|c|c|c|c|}
\hline Phantom & AlSPc & Photofrin & DCM & $\begin{array}{c}\text { Fluorescein } \\
\text { Inulin }\end{array}$ & $\begin{array}{c}\text { Ethidium } \\
\text { Bromide }\end{array}$ & $\begin{array}{c}\text { Flazo } \\
\text { Orange }\end{array}$ \\
\hline Liquid & $\mathrm{F}$ & $\mathrm{F}$ & $\mathrm{F}, \mathrm{B}^{2}$ & $\mathrm{~F}, \mathrm{~B}^{2}$ & $?$ & $\mathrm{~F}^{3}$ \\
\hline Resin & $\mathrm{F}$ & $\mathrm{F}, \mathrm{B}^{1}$ & $\mathrm{~F}, \mathrm{~B}$ & $\mathrm{~F}, \mathrm{~B}$ & $\mathrm{~F}, \mathrm{~B}$ & $?$ \\
\hline Silicone & $\mathrm{F}$ & $\mathrm{F}$ & $\mathrm{F}, \mathrm{B}$ & $\mathrm{F}, \mathrm{B}$ & $?$ & $?$ \\
\hline Agar & $\mathrm{F}$ & $\mathrm{F}$ & $?$ & $?$ & $?$ & $?$ \\
\hline
\end{tabular}

Table 1: Experimentally determined fluorophore properties for various phantom materials $(\mathrm{F}=$ Fluorescent emission, $\mathrm{B}=$ Bleaches significantly). The liquid phantom was a typical Travamulsion ${ }^{\circledR}$ solution, and the resin refers to the clear casting polystyrene resin. For further information, see Appendix 1: Phantom Preparation, as well as Appendix 2: Fluorophores.

'Bleaching observed in small phantom's which do not reach high curing temperatures.

${ }^{2}$ Assumed to bleach in liquid.

${ }^{3}$ Fluoresces in a Methanol solution, not water. 


\subsection{Characterization of Drug}

The Life Science UV/Vis Spectrophotometer (Beckman $\mathrm{DU}^{\circledR}$ 530, single cell module) was used to measure the optical absorbance for the dilution series of the $\mathrm{AlS}_{4} \mathrm{Pc}$. The absorbance at the excitation and emission wavelengths was measured across the 10 mm path length of each cuvette. At larger drug concentrations (on the order of $1 \mu \mathrm{g} / \mathrm{ml}$ and greater), the relationship between the absorbance and concentration is non-linear; it is thought that this is due to aggregation of the fluorophores [see Figure 8]. As the solution is diluted, the probability of reabsorption diminishes, and this relationship becomes linear. The absorbance can then be fitted for a number of dilutions, allowing the accurate determination of the optical absorbance (base 10). Multiplying this value by $\ln (10)$ yields the absorption coefficient (base e), which is then used for the diffusion theory calculations. Both the optical absorbance and absorption coefficient have dimensions of $\mathrm{mm}^{-1}(\mu \mathrm{g} / \mathrm{ml})^{-1}$, so the appropriate designation is important to avoid an error by the factor of $\ln (10)$.

A problem with this method of drug characterization is that the properties of the drug (absorption, branching ratios, fluorescent yield, etc.) are often altered by its physicochemical environment. Consequently there is no guarantee that these properties will remain consistent in our tissue equivalent phantoms. Furthermore, the degree of photosensitizer coupling is unknown, so the bleach rate may not be a useful indication of the photodynamic effect. 


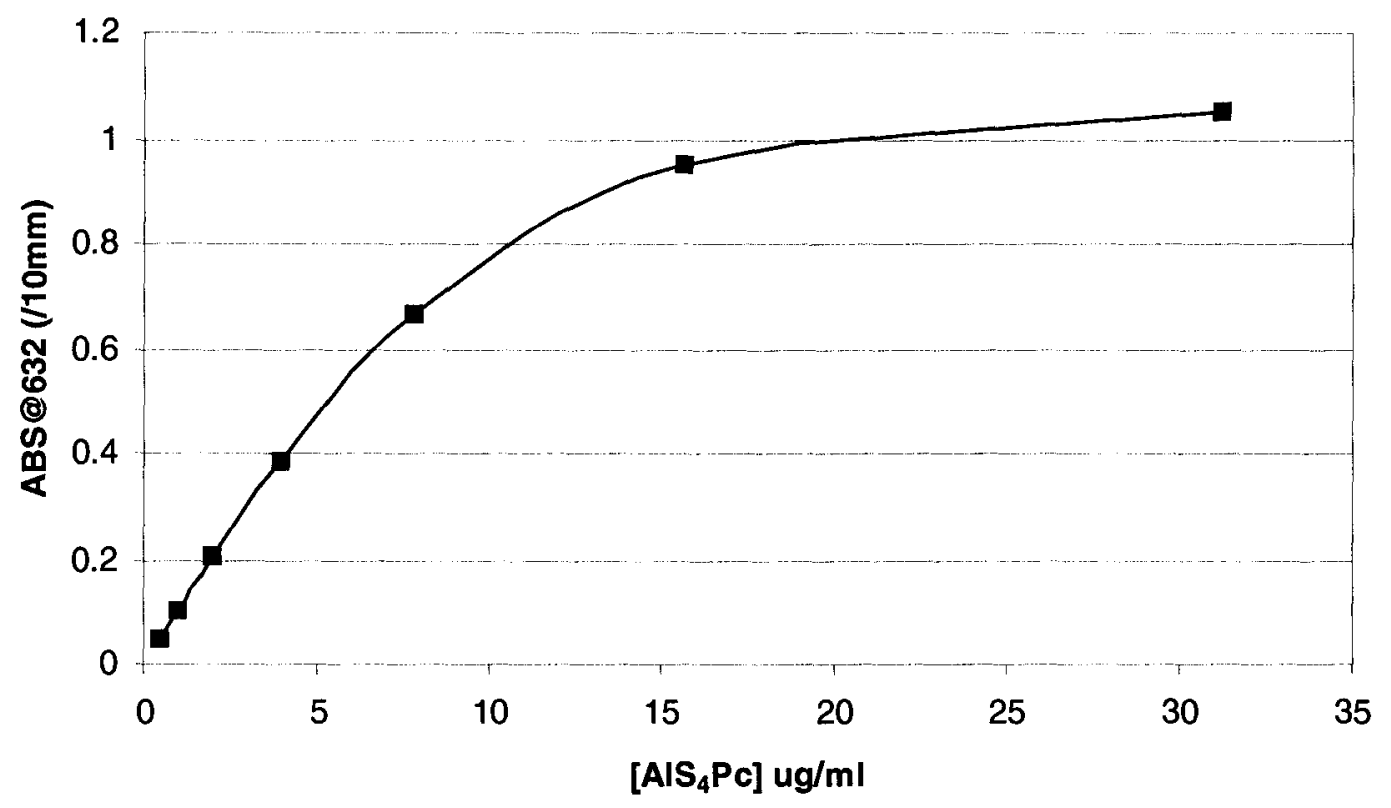

Figure 8: Aluminum tetra-Sulphonate Pthalocyanine absorbance at the excitation wavelength $(632 \mathrm{~nm})$ in $0.8 \%$ Phosphate Buffered Saline solution. Note the non-linear relationship at higher concentrations, which is thought to be caused by the aggregation of the fluorophores. Clinical concentrations are generally less than $5 \mu \mathrm{g} / \mathrm{ml}$ (as in our experiments), which is basically within the linear regime. 
This procedure was performed for aluminum pthalocyanine tetra-sulphonate $\left(\mathrm{AlS}_{4} \mathrm{Pc}\right.$ ) and the peak optical absorbance (at $675 \mathrm{~nm}$ ) was determined to be $0.0193 \mathrm{~mm}^{-}$ ${ }^{1}(\mu \mathrm{g} / \mathrm{ml})^{-1}$. This corresponds to an absorption coefficient of $0.0445 \mathrm{~mm}^{-1}(\mu \mathrm{g} / \mathrm{ml})^{-1}$, which is in agreement with the value of $0.0435 \mathrm{~mm}^{-1}(\mu \mathrm{g} / \mathrm{ml})^{-1}$, which was previously determined using a different method, see Figure 9. [Patterson, 1994]

For the experimental results which follow, a stock solution of $\mathrm{AlS}_{4} \mathrm{Pc}$ was produced with a concentration of $500 \mu \mathrm{g} / \mathrm{ml}$. Using the above protocol, the absorption coefficient at $632 \mathrm{~nm}$ (the experimental excitation wavelength) was determined to be $0.00599 \mathrm{~mm}^{-1}(\mu \mathrm{g} / \mathrm{ml})^{-1}$. Similarly, the absorption coefficient at $683 \mathrm{~nm}$ (the peak fluorescence emission) was found to be $0.02496 \mathrm{~mm}^{-1}(\mu \mathrm{g} / \mathrm{ml})^{-1}$. These measurements were performed on the day of the experiment so that the properties of the photosensitizer could be accurately determined (the photodynamic activity decreases over time).

\subsection{Layered Phantoms}

Creating phantoms with a varying drug distribution, to simulate the diffusion of drug into the tissue and the ensuing photo-bleaching, is extremely difficult. Thus, the diffusion has been simplified by modeling finite layer thicknesses, of a known homogeneous drug concentration, at the surface of a semi-infinite tissue equivalent medium (Figure 10). Although not the general case, this situation could be used to describe the steady state drug distribution at a diffusion barrier. Regardless, the dose delivered to regions close to the site of irradiation will be limited by the photo-bleaching 


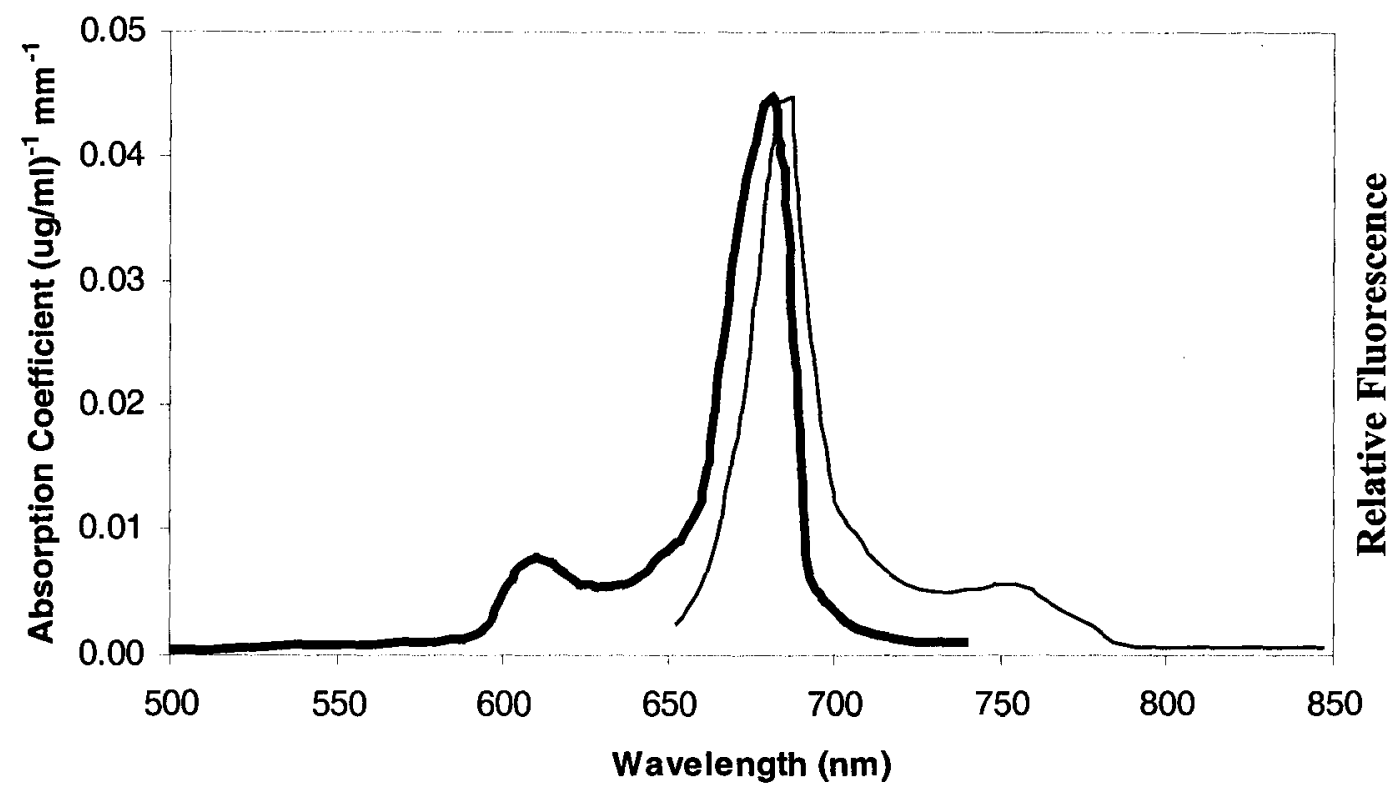

Figure 9: Aluminum pthalocyanine tetra-sulphonate $\left(\mathrm{AlS}_{4} \mathrm{Pc}\right)$ absorption spectrum (thick line), and relative fluorescence spectrum (thin line). [Patterson, 1994] 
of the drug, rather than by the optical dose. Consequently, the accurate determination of this drug concentration is a crucial element of the dose assessment.

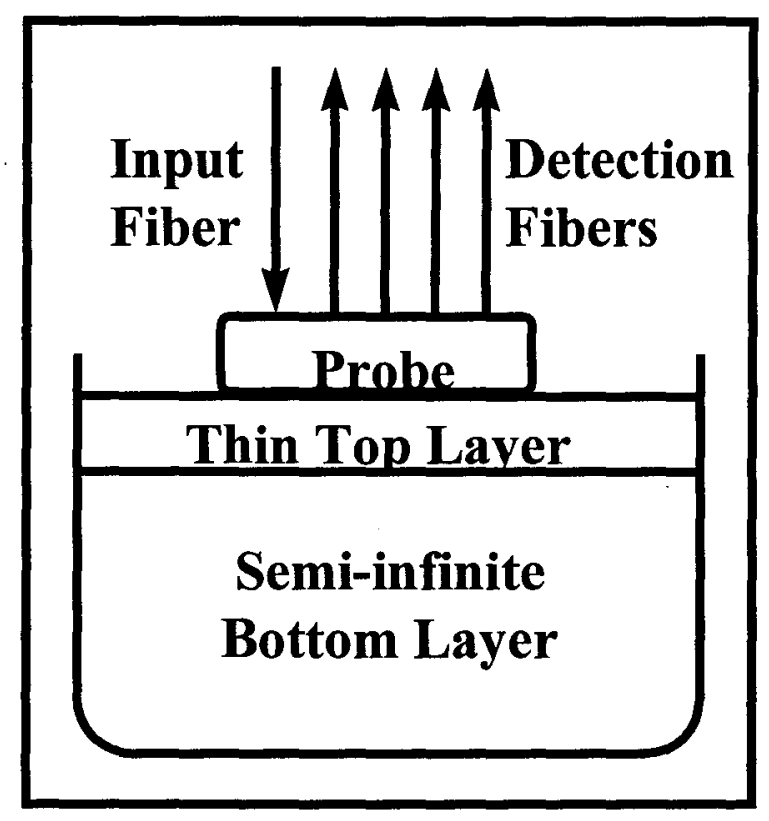

Figure 10: Detection of Spatially Resolved Fluorescence

Similarly, the photo-bleaching is modeled by thin layers of a tissue equivalent medium at the top of one which contains drug. Although this is a crude approximation to what one would observe clinically, it does demonstrate the effectiveness of our spatially resolved fluorescence measurements. However, despite this simplified geometry, there were still a number of intricacies in the phantom development.

When constructing a layered phantom with more than one type of scattering material, it must be remembered that although the scattering may be equal at the excitation wavelength, it probably isn't at the fluorescence emission wavelength. This is 
simply due to the wavelength dependence of the scatterer (scattering decreases with wavelength when using $\mathrm{TiO}_{2}$, but Intralipid ${ }^{\circledR}$ or Travamulsion ${ }^{\circledR}$ have a more complex relationship due to their various constituents). This must be taken into account because the optical properties, at the wavelengths of interest, should be closely matched to minimize layer boundary effects.

When constructing a layered medium, it must also be remembered that the settling of any of the constituents will yield a non-homogeneous layer. In addition, when using two different phantom materials, any index of refraction mismatch must also be taken into account. Finally, if photobleaching is to be analysed, the phantom must exist in a relatively solid form in order to prevent the mixing of the various constituents.

A number of layered phantoms were constructed with varying degrees of success. Liquid layers were poured on top of solid silicone and polystyrene resin phantoms, but the differing scatter coefficients produced boundary effects which were difficult to model. Liquid was used on top of an agar phantom, but the structure of the agar allowed a small amount of drug to diffuse between the two layers. A polystyrene layer was cast on top of a polystyrene resin phantom which had been sanded to a smooth plane. This produced two discernible layers, but the $\mathrm{TiO}_{2}$ settling produced inhomogeneous layers (with a large number of scatterers at the layer's interface), which were difficult to accurately model. Consequently, these difficulties lead to the use of a liquid-liquid phantom. 


\subsection{Liquid-Liquid Phantom}

Two liquid layers were separated with an optically transparent sheet of plastic 'sandwich' wrap (see Figure 11), which typically has a thickness of $10.668 \mu \mathrm{m}$, and a refractive index of 1.523 [Rider, 1999]. There wasn't any significant boundary effect observed (see Figures $12 \&$ 13). Travamulsion ${ }^{\circledR}$ and India ink were used to adjust the scattering and absorption characteristics of the medium.

The entire phantom was placed on a micro-adjustable vertical positioning stage. The smaller top container was placed on its stand with the plastic sheet in place. The larger container was then filled to the level of the plastic sheet (without trapping any air), and the probe was suspended at the surface of the sheet. This was used as the reference point, such that the top layer thickness equaled zero. The top layer solution was then added to the smaller container, and an equal volume of the other solution was added to the large container. This ensured that the pressure on each side of the plastic sheet was equal, and that it was not forced away from the reference point. As drug was added to one of the layers, it was stirred into a homogeneous solution using a plastic ruler. An equal volume of fluid was then added to the other layer to equate the hydraulic pressure. 


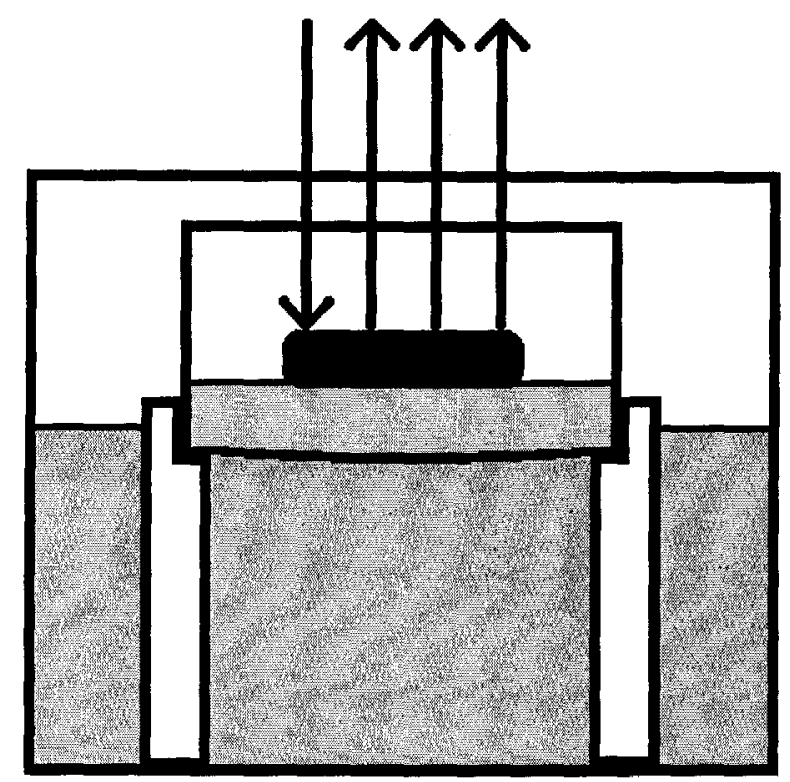

Figure 11: Schematic diagram of a two layer liquid phantom. The bottom layer (large container) has optical properties similar to that of tissue (actual absorption not as high as it may appear). The top layer (smaller container) has $\mathrm{AlS}_{4} \mathrm{Pc}$ added to a portion of liquid from the bottom layer, and it is separated by a container with an optically transparent sheet of plastic (PVC) 'sandwich' wrap as its base. It should be noted that if the liquid levels are not equal, the pressure will stretch the thin plastic into a concaved shape (as seen above). A black probe, which consists of one source fibre and a number of detection fibres, is suspended at the surface. 


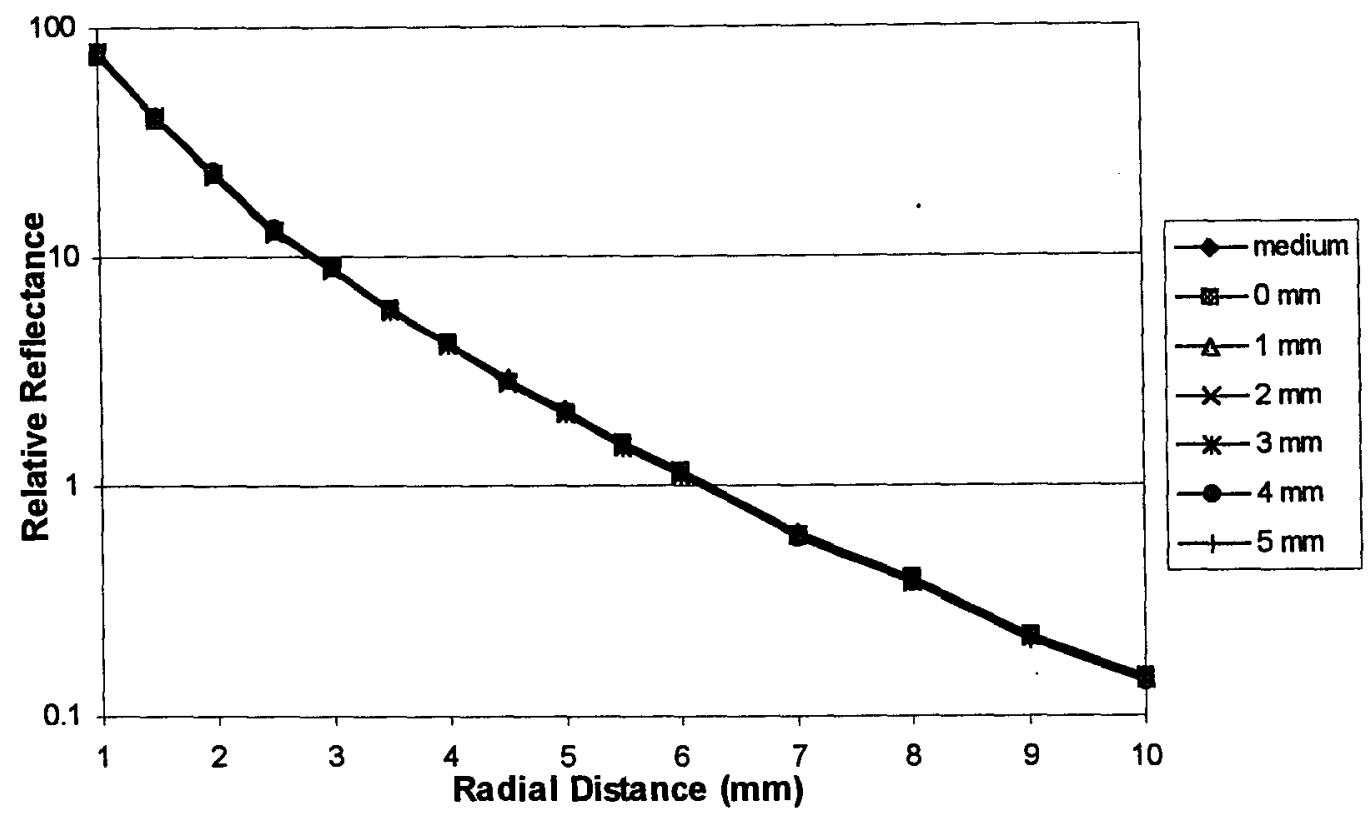

Figure 12a: The spatially resolved reflectance from a tissue equivalent liquid, as well as the reflectance from two liquid layers (separated by transparent plastic wrap) with a variety of top layer thicknesses.

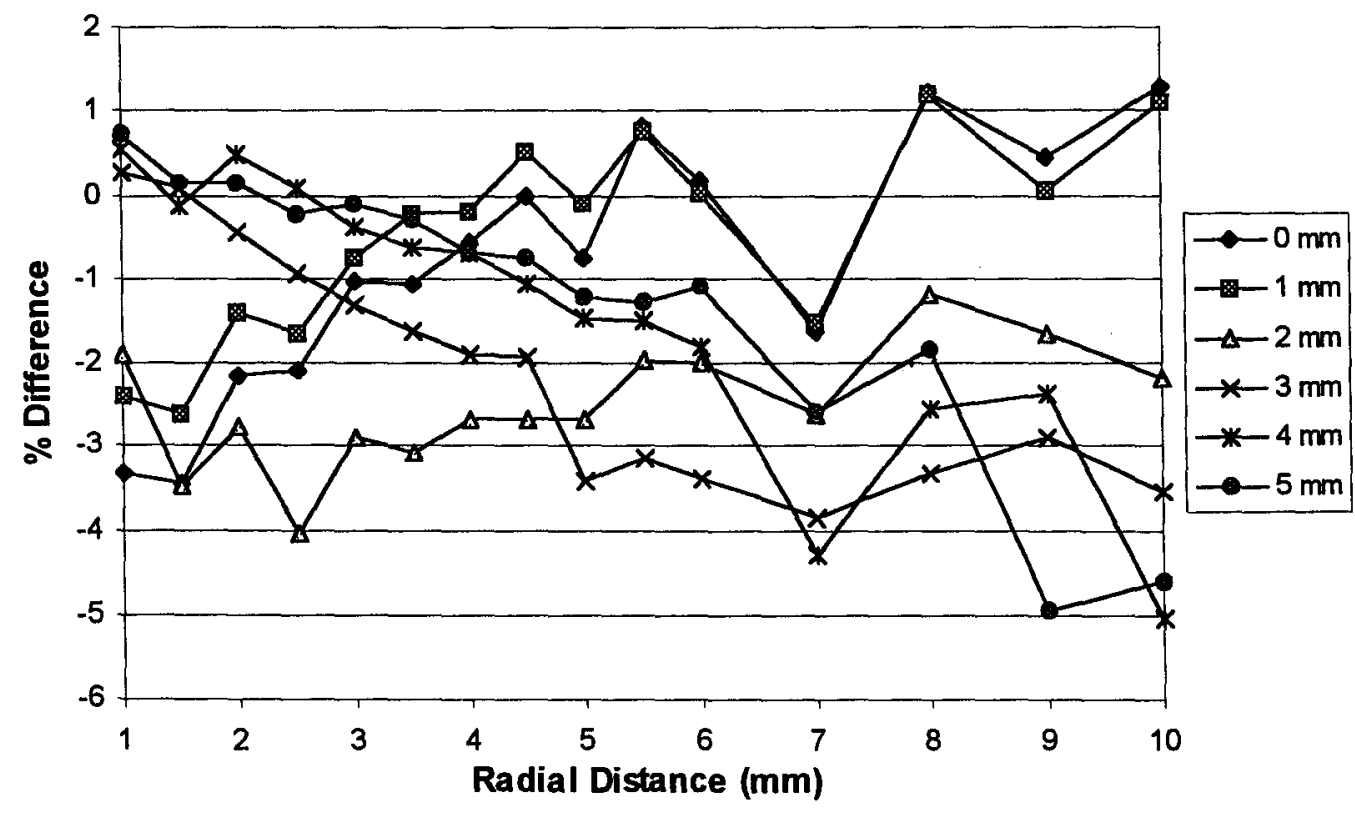

Figure 12b: A plot of the percentage difference of the various layers from the medium itself (spline interpolation between points). This shows that there is no significant trend to the data. 


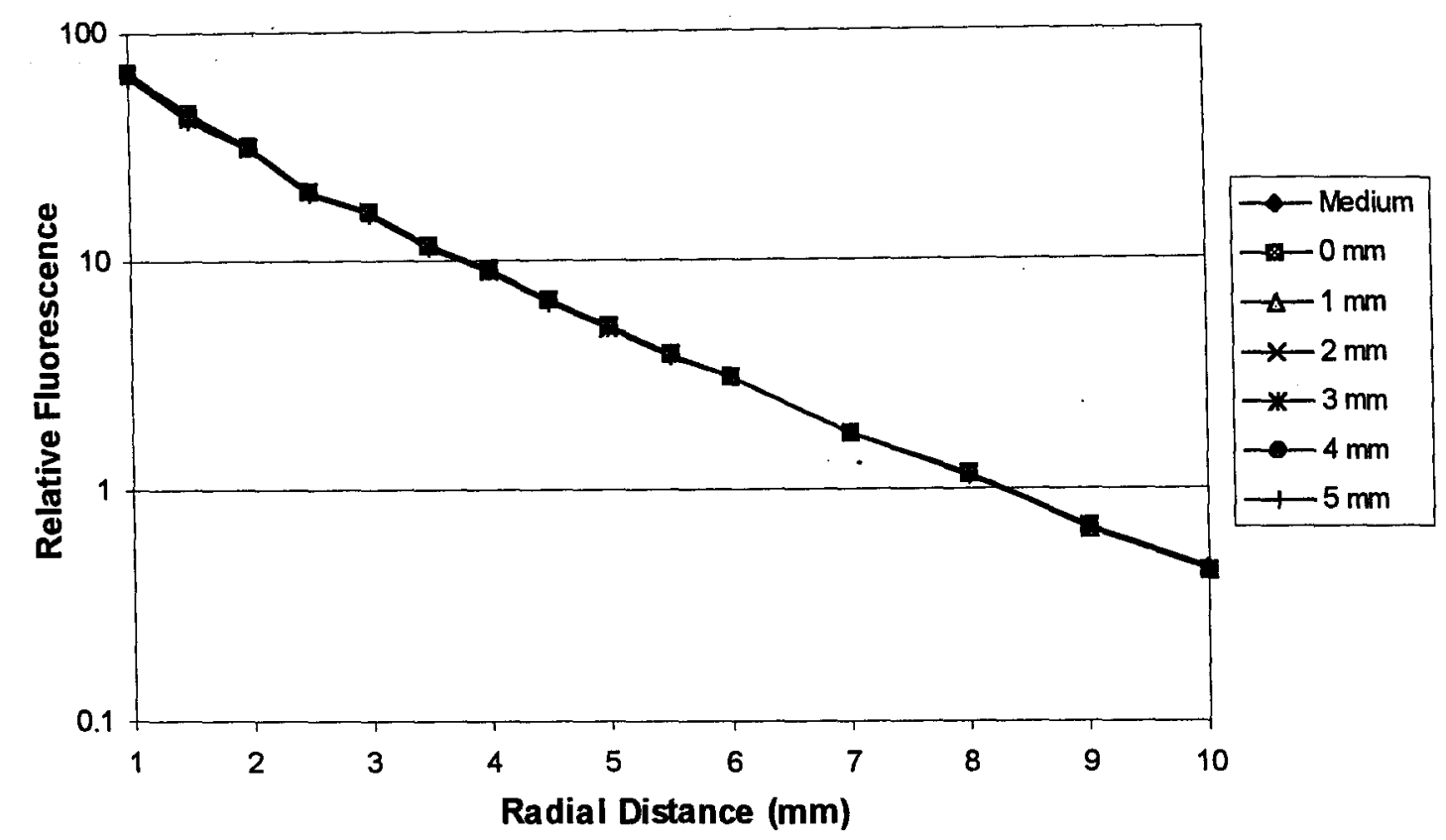

Figure 13a: The spatially resolved fluorescence from a tissue equivalent liquid, as well as the fluorescence from two liquid layers (separated by transparent plastic wrap) with a variety of top layer thicknesses.

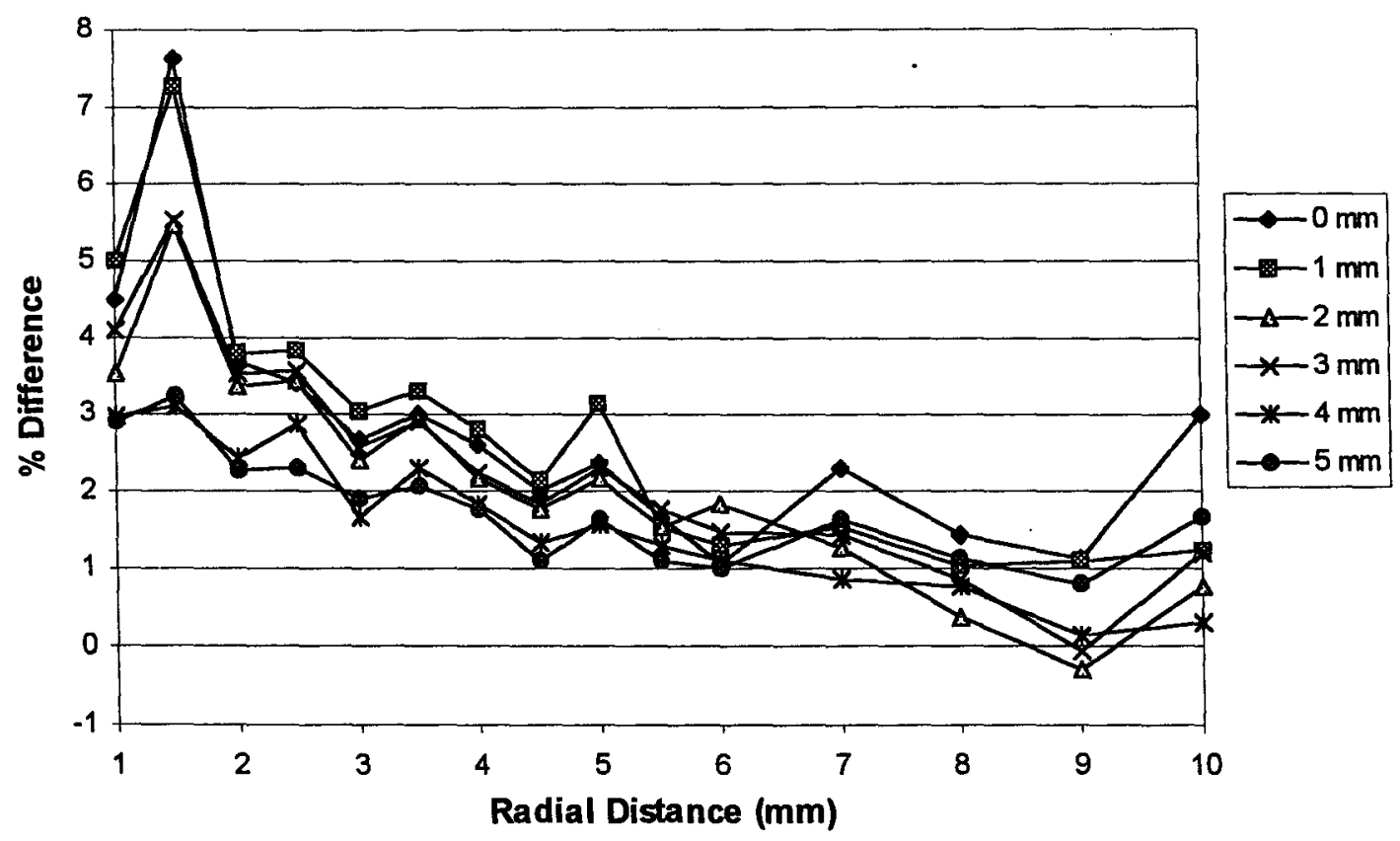

Figure 13b: A plot of the percentage difference of the various layers from the medium itself (spline interpolation between points). This shows that the plastic wrap produces a small increase in the fluorescence intensity, particularly at the smaller radial distances. 


\subsection{The Mark II System}

The Mark II system is set up for the acquisition of steady-state spatially resolved diffuse reflectance/fluorescence. The various components of the system are shown in Figure 14.

Although any light source may be coupled to the probe via a SMA connector, an Oriel Instruments ${ }^{\circledR}$ broadband quartz tungsten halogen (QTH) source is devoted to this system. It is equipped with a variable current power supply, an electronic shutter, and a photofeedback system to stabilize the light intensity. Neutral density, bandpass, or cut-on filters can be placed in the collimated output source.

The black Fibreguide ${ }^{\circledR}$ probe (assumed to be a perfectly absorbing medium) consisted of two sets of fifteen detection fibres which were located at increasing radial distances ( 1 to $10 \mathrm{~mm}$ ) from a single source fibre. The source fibre provided the pencil beam excitation, and one detection fibre set was used to measure the spatially resolved fluorescence. The fibres which were close to the source were attenuated $\left(\mathrm{OZ}\right.$ optics $\left.{ }^{\circledR}\right)$ so their intensity would not saturate the charged coupled device (CCD). Consequently, a calibration is required to determine the relative intensity of each of the fibres.

A Fibreguide ${ }^{\circledR}$ coupling piece vertically aligns the fibres (after passing through the variable attenuators) in a linear arrangement for imaging onto the CCD. The fibres located at the larger radial distances were placed in the center to improve the sensitivity, allowing the detection of the lower intensities. The fibres are generally separated by a single fibre width so that their image is clearly resolvable. A spectrometer (Kaiser ${ }^{\mathbb{Q}}$ ) then provides wavelength dispersion of the detected light in the horizontal plane. 


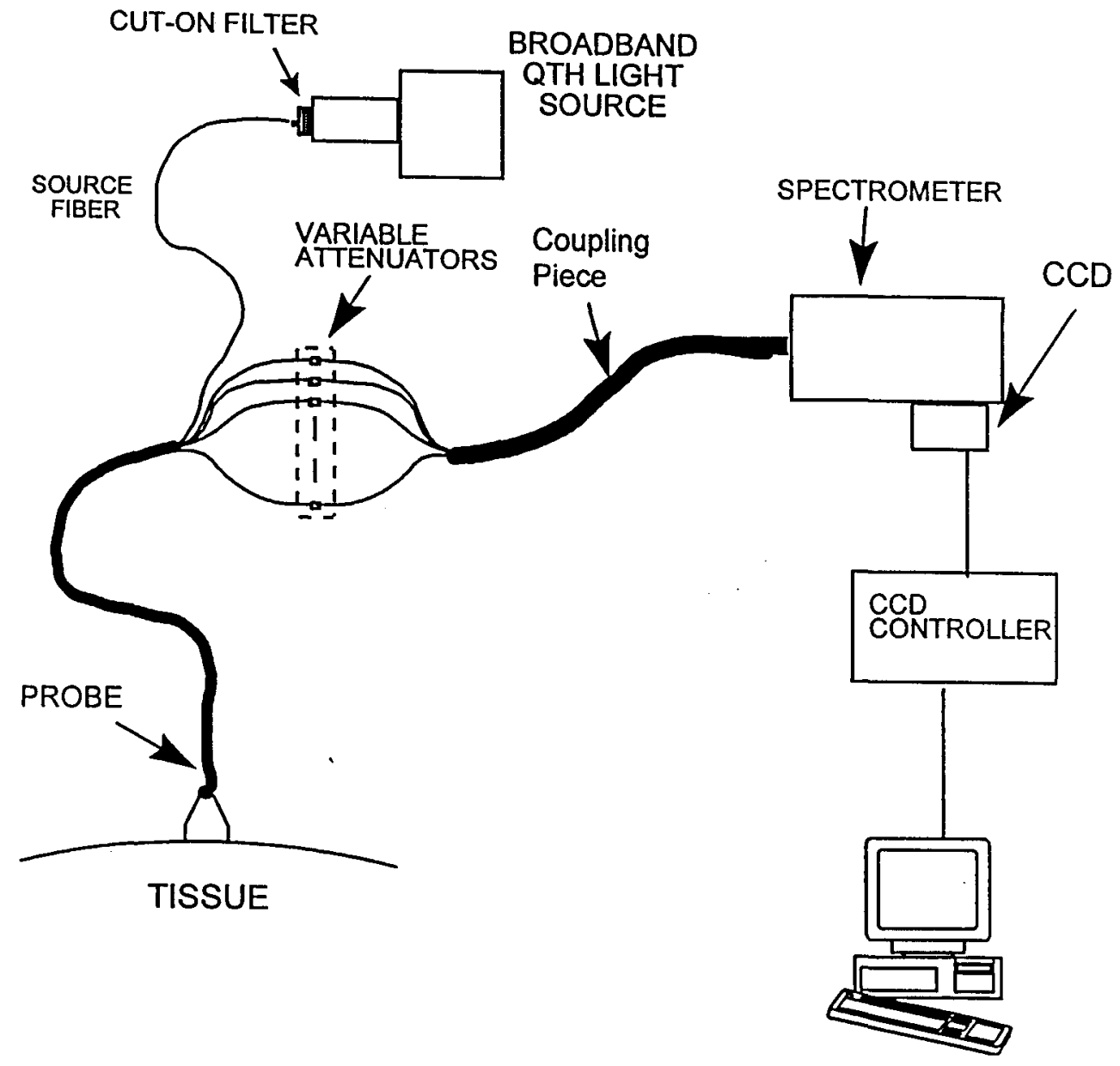

Figure 14: Principle Components of the Mark II System [Bruulsema, 1997] 
The Princeton Instruments ${ }^{\circledR}$ CCD (EEV $1152 \times 1242,6 \mathrm{ph}$ ) is a two dimensional array which measures the intensity of the incident light. The (ST138) controller is responsible for the electronic shutter, intensity readout, and the thermoelectric cooling to minimize the dark counts. Each fibre is then imaged as a horizontal band, providing the intensity at all wavelengths. Winspec ${ }^{\circledR}$ v1.4.1 (Princeton Instruments ${ }^{\circledR}$ ) spectroscopy software was used to operate the controller and analyse the images.

\subsection{Measurement Technique}

An appropriate cut-on filter was chosen so that the reflected excitation light would not saturate the $C C D$, but at the same time, the attenuation of the fluorescence was minimal. This filter was placed at the fibre optic output where it is coupled to the CCD array, and was not moved for the duration of the experiment. All images were collected, stored and displayed using Winspec ${ }^{\circledR}$. The exposure time was adjusted to prevent saturation, and the 15 fibre tracks were imaged and subsequently binned according to their placement on the CCD array. A small cross-talk correction track was also binned between each fibre track, allowing the subtraction of any unwanted photons.

The probe was then placed into a calibration sphere which uniformly scatters light, providing a homogeneous intensity to each of the tracks. The QTH broad band light source was then coupled to a separate BNC connection on the calibration sphere. It is important not to use the source fibre of the probe because specular reflection from the opposite wall of the sphere may corrupt the calibration procedure (since each track would no longer receive an equal amount of light from the source). The aperture on the light 
source was adjusted to provide an intensity which was suitable for an exposure time of at least one second (to minimize any error caused by the shutters action). After the background exposure (dark counts), an exposure of the calibration sphere was taken to form the calibration file.

The probe was then removed from the calibration sphere, and placed on the surface of the phantom. The source fibre from the probe was then connected to the desired light source. The intensity of this source was also adjusted to provide an exposure time of at least one second. The background and actual exposure were taken and the calibration file was used to determine the relative intensities from each of the tracks.

The optical properties were determined for the phantoms which did not contain drug. This involved the measurement of the spatially resolved reflectance, from the broad band light source, with the Mark II system. The reflectance was then interpreted by comparison to previously acquired data with known optical properties (determined by Mie theory). Human skin generally has absorption and reduced scattering coefficients of approximately 0.01 and $1.5 \mathrm{~mm}^{-1}$ respectively [Bruulsema, 1997]. Therefore, to model a treatment with topically administered photosensitizers, the optical properties of the liquid were adjusted (by varying the concentration of Travamulsion ${ }^{\circledR}$ and India ink) such that $\mu_{s^{\prime}(@ 632 \mathrm{~nm})}^{\prime}=1.44 \mathrm{~mm}^{-1}, \mu_{s^{\prime}(663 \mathrm{~nm})}=1.14 \mathrm{~mm}^{-1}$, and $\mu_{\mathrm{a}}=0.013 \mathrm{~mm}^{-1}$ for both wavelengths. 


\section{RESULTS}

\subsection{Drug in Bottom Layer}

\section{Diffusion Theory and Monte Carlo}

The measured optical properties of the liquid phantom were used in this theoretical comparison as well. The fluorescence intensities were originally normalized to the probability for a single photon, but it was found that the model consistently produced slightly lower fluorescence intensities, which appeared to vary by a single scaling factor $(\sim 0.7575)$. Consequently, each data set was normalized to a point in the middle of the set $\left(\left[\mathrm{AlS}_{4} \mathrm{Pc}\right]\right.$ of $2 \mu \mathrm{g} / \mathrm{ml}, 3 \mathrm{~mm}$ top layer thickness, and a radial distance of $5 \mathrm{~mm}$ ). After this intensity adjustment, it was clear that the shapes of the Monte Carlo and diffusion theory curves agreed quite well,

Top layer thicknesses $(1,2,3,4$ and $5 \mathrm{~mm})$ were analysed for clinically appropriate drug concentrations of $0.5,1,2,4$ and $6 \mu \mathrm{g} / \mathrm{ml}$ (see Figures 15a-e). The five drug concentrations are clearly discernible at each of the measured depths. As the fluorophore concentration was increased, the intensities for the smaller radial distances increased, thereby increasing the slope of the spatially resolved fluorescence curves. For larger concentrations, this increase in slope caused the curve to cross over those of lower concentrations (within the radial distance of $10 \mathrm{~mm}$ ). 


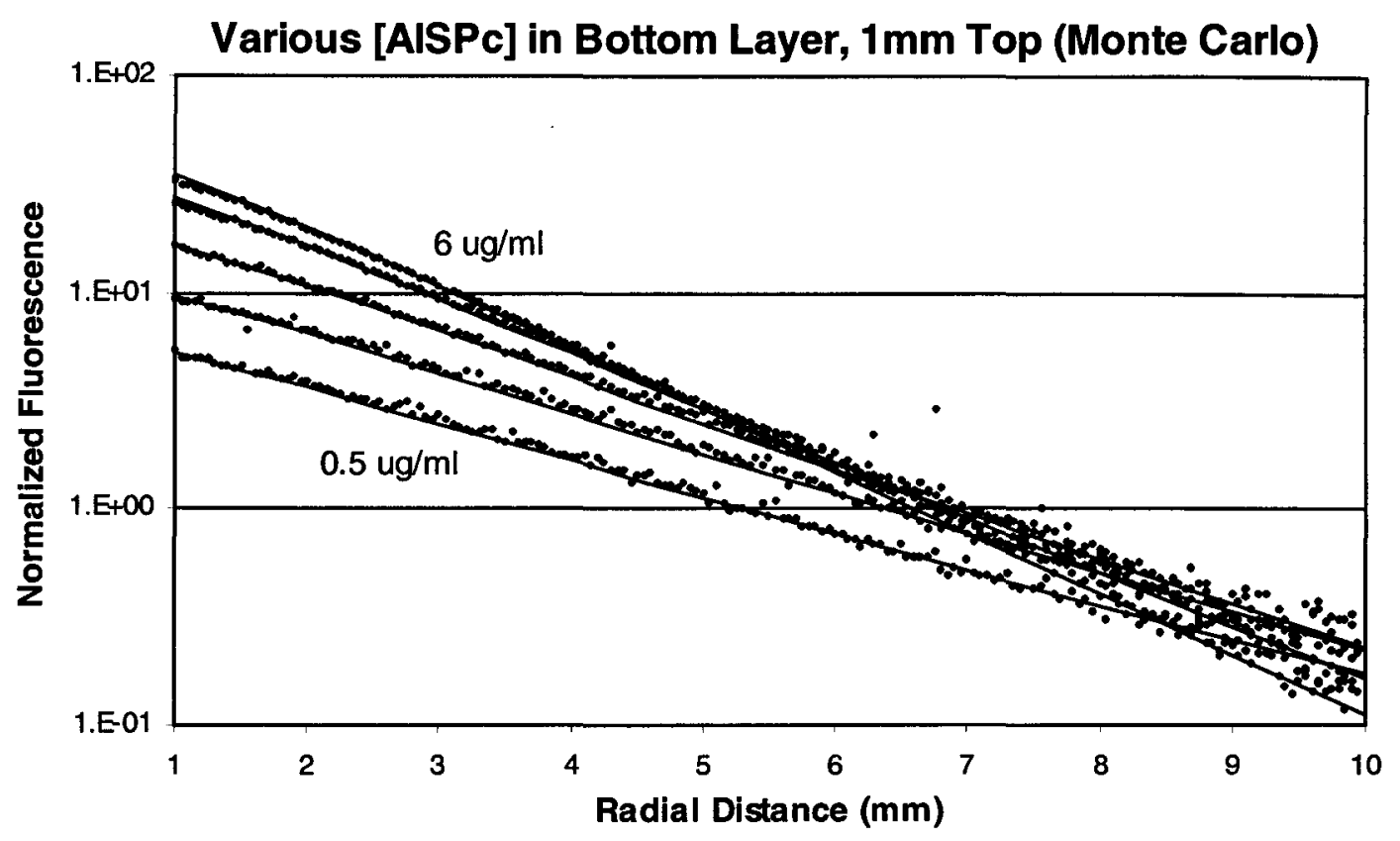

Figure 15a: Diffusion theory (lines) and Monte Carlo (points) for $0.5,1,2,4$ and $6 \mu \mathrm{g} / \mathrm{ml}$

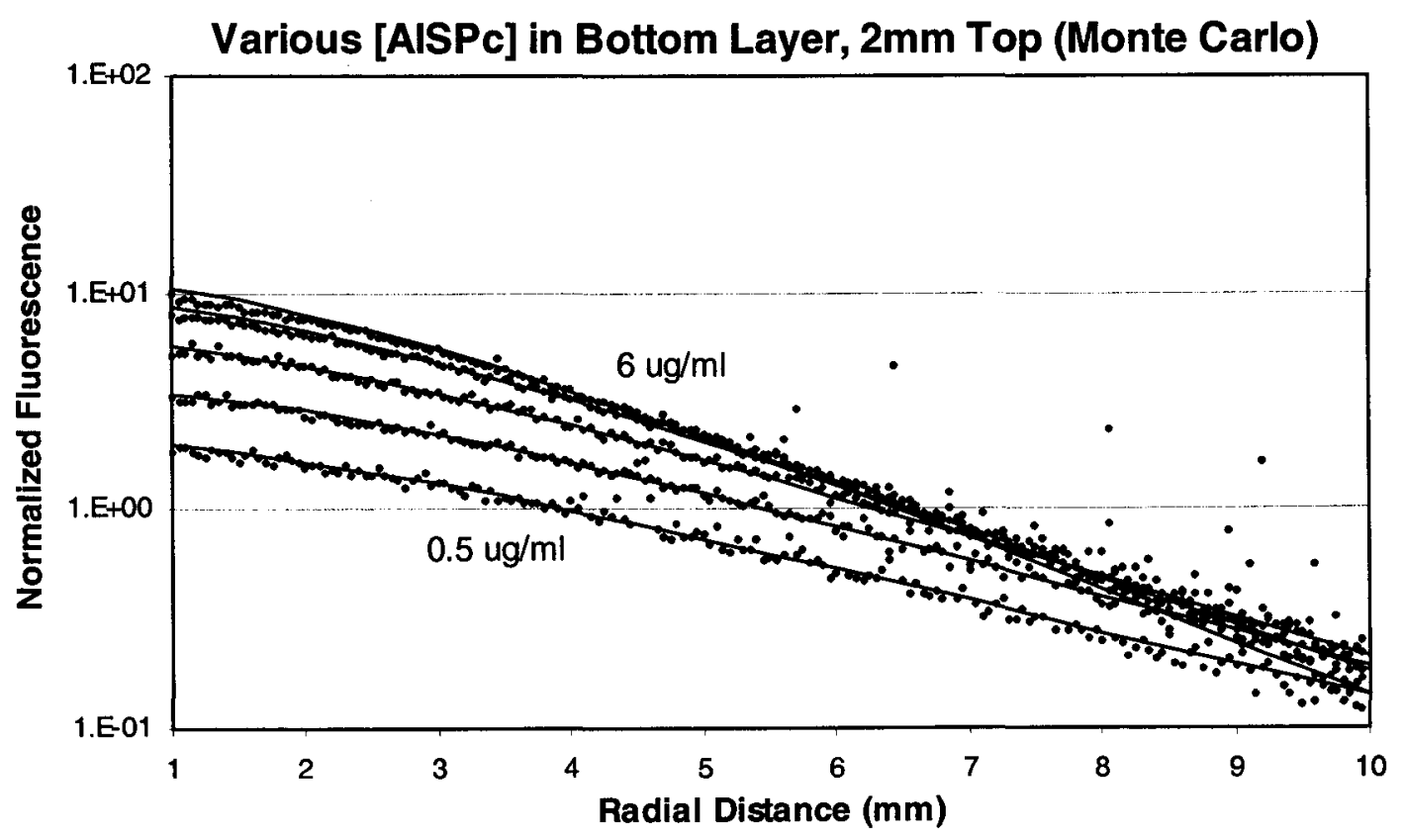

Figure 15b: Diffusion theory (lines) and Monte Carlo (points) for $0.5,1,2,4$ and $6 \mu \mathrm{g} / \mathrm{ml}$ 


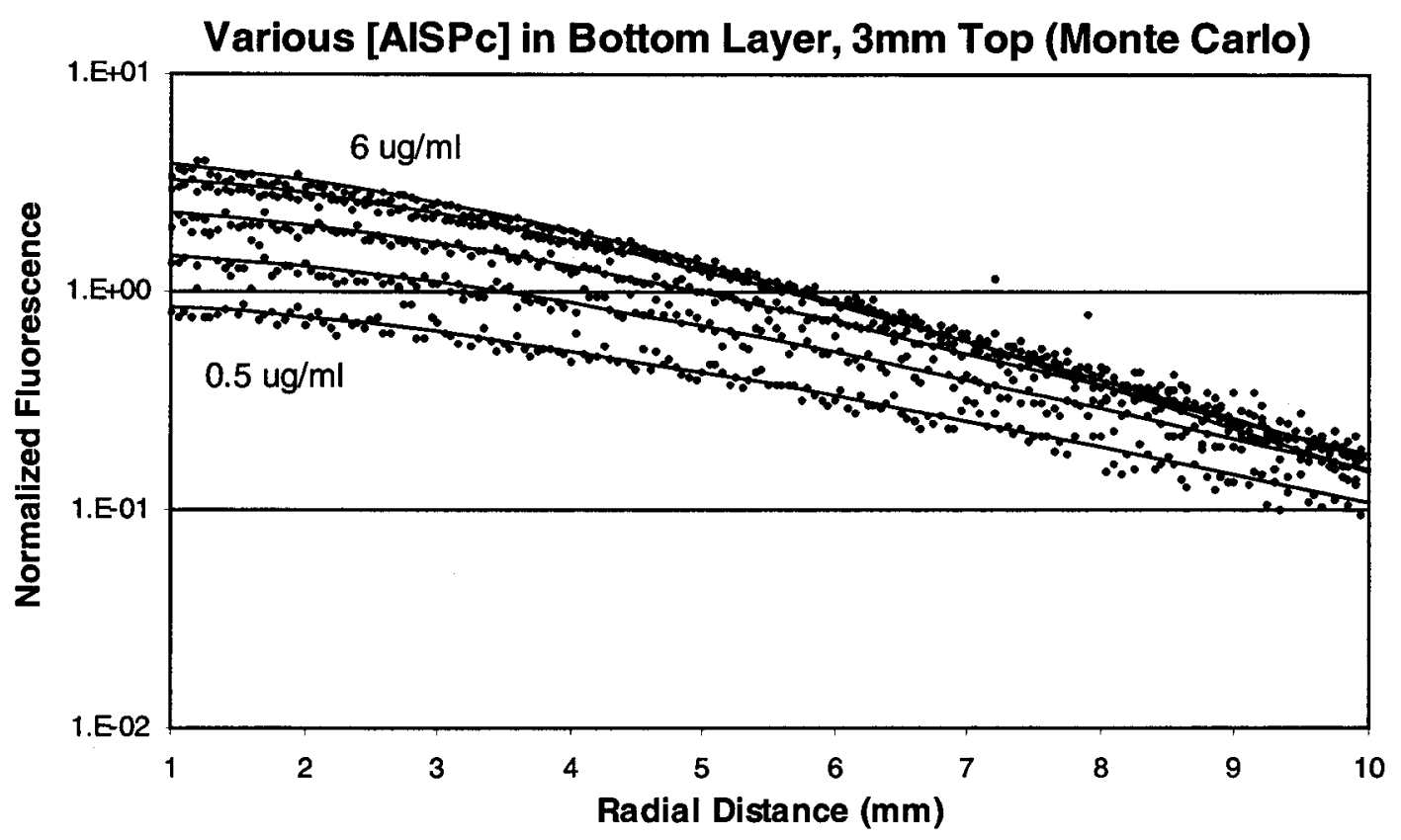

Figure 15c: Diffusion theory (lines) and Monte Carlo (points) for $0.5,1,2,4$ and $6 \mu \mathrm{g} / \mathrm{ml}$

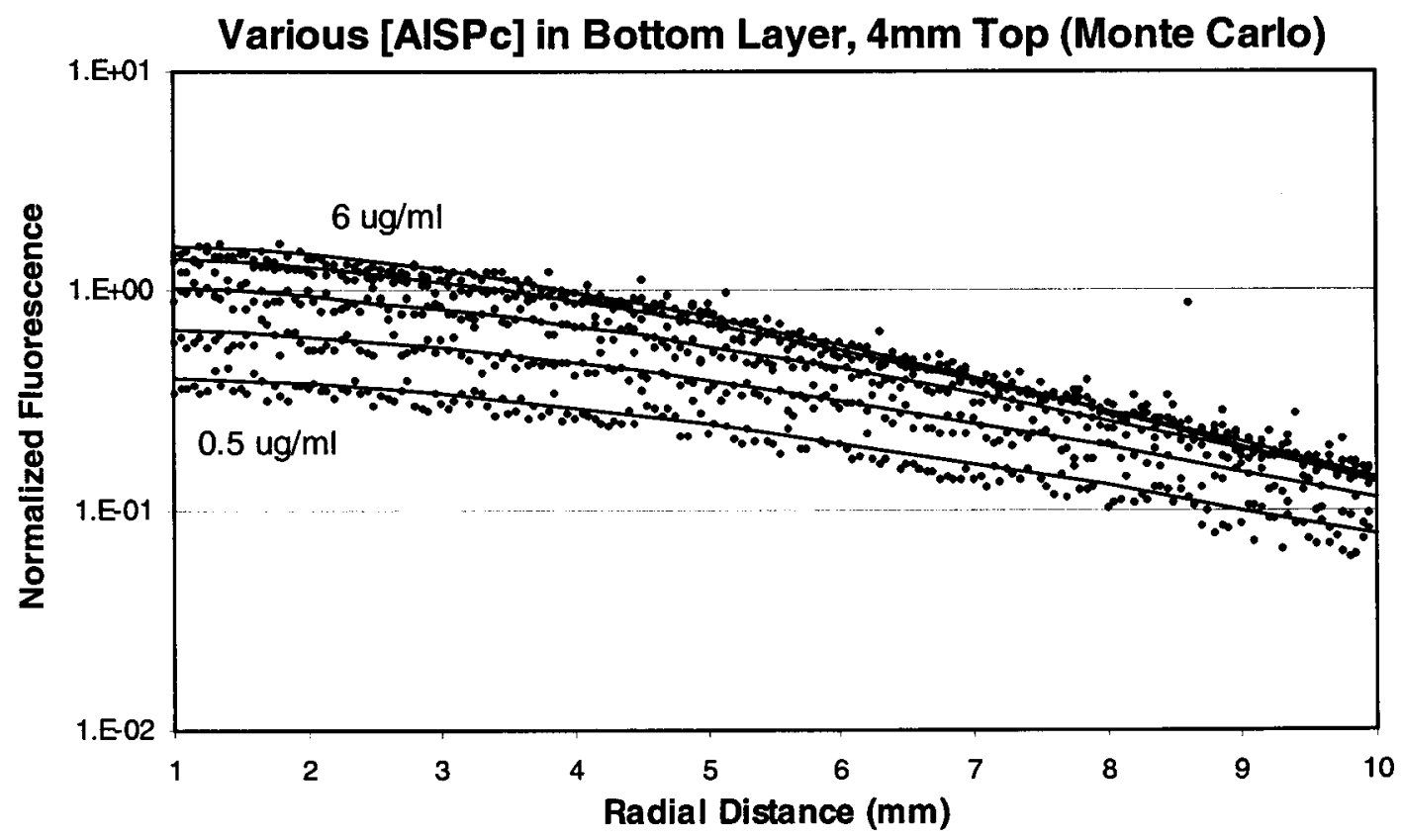

Figure 15d: Diffusion theory (lines) and Monte Carlo (points) for $0.5,1,2,4$ and $6 \mu \mathrm{g} / \mathrm{ml}$ 


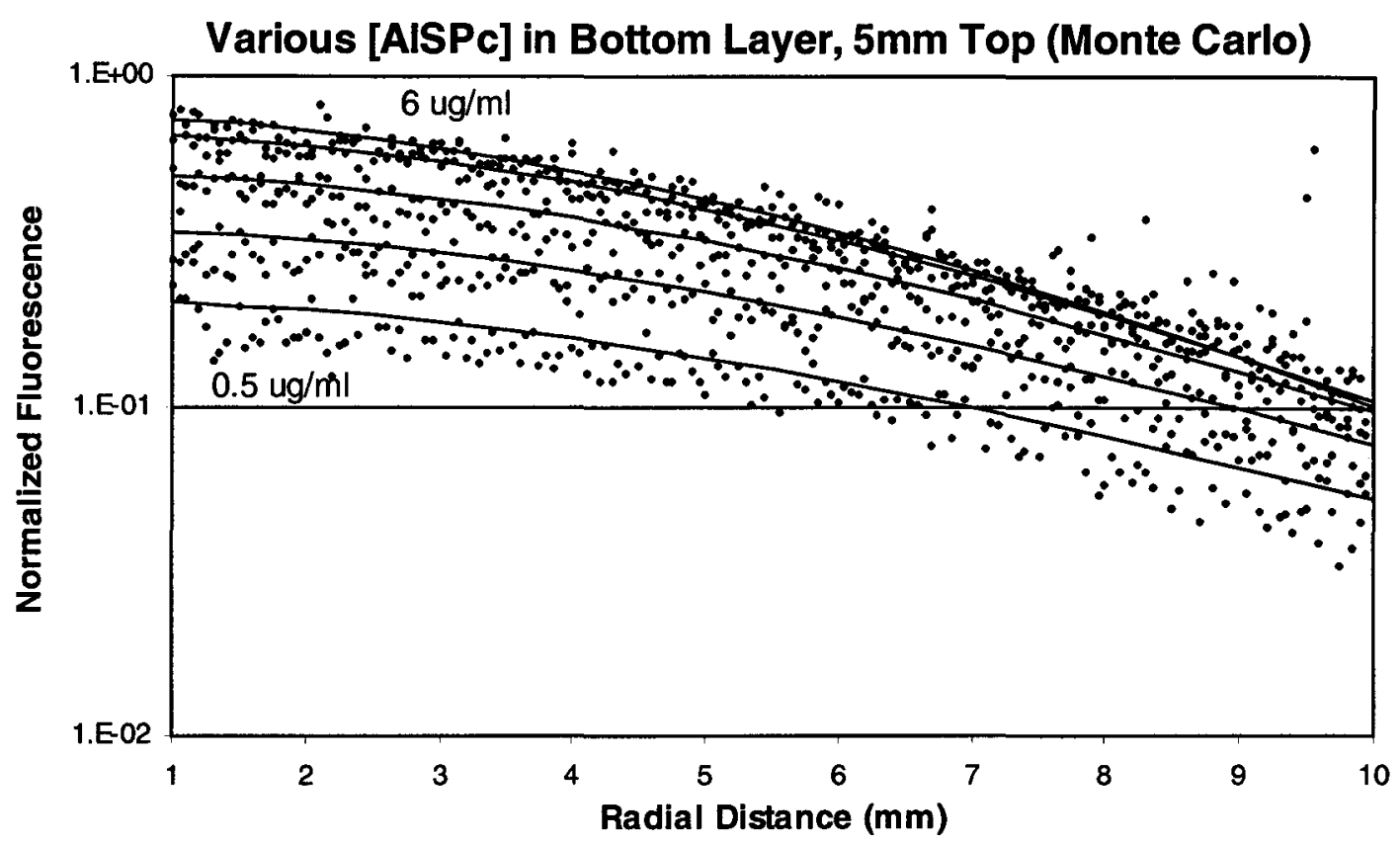

Figure 15e: Diffusion theory (lines) and Monte Carlo (points) for $0.5,1,2,4$ and $6 \mu \mathrm{g} / \mathrm{ml}$

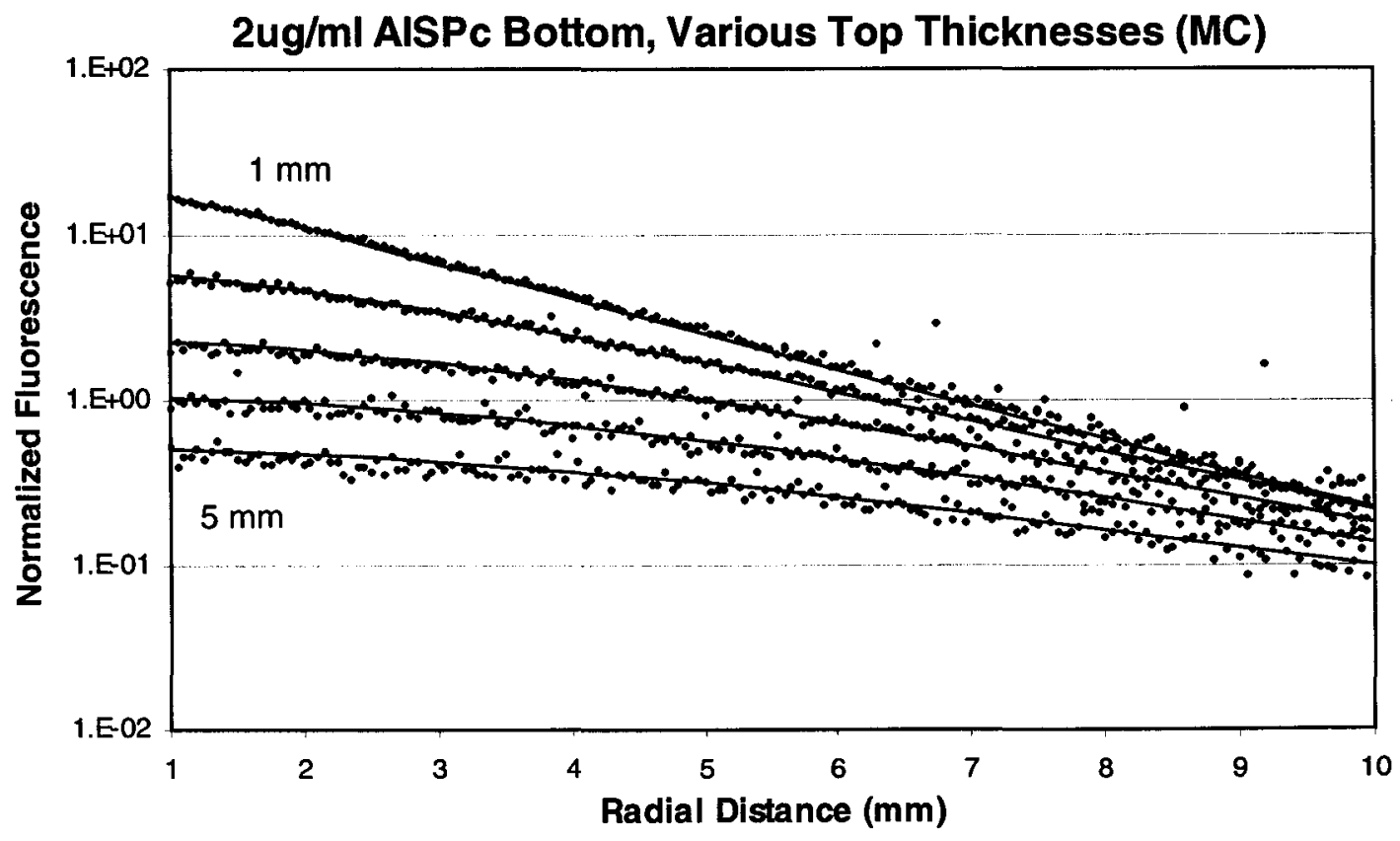

Figure 15f: Diffusion theory (lines) and Monte Carlo (points) for $0.5,1,2,4$ and $6 \mu \mathrm{g} / \mathrm{ml}$ 
Each top layer thickness was also plotted for an $\mathrm{AlS}_{4} \mathrm{Pc}$ concentration of $2 \mu \mathrm{g} / \mathrm{ml}$ to illustrate the ability to differentiate the drug depth (see Figure 15f). Note that both the intensity and slope decreased as the depth of the fluorophore increased.

\section{Diffusion Theory and Experimental Measurements}

A royal lavender filter was used to prevent the $632 \mathrm{~nm}$ excitation light (HeliumNeon laser) from saturating the CCD. Due to the experimental difficulty in measuring the source strength, a relative comparison was performed. A point from the middle of the data set was used for the normalization of the measurement to the theory $\left(\left[\mathrm{AlS}_{4} \mathrm{Pc}\right]\right.$ of $2 \mu \mathrm{g} / \mathrm{ml}, 3 \mathrm{~mm}$ top layer thickness, and a radial distance of $5 \mathrm{~mm}$ ), and all of the fluorescence intensities were normalized to that point. The experimental measurements were then plotted with the Diffusion theory for 1 to $5 \mathrm{~mm}$ top layer thicknesses and drug concentrations of $0.5,1,2$ and $4 \mu \mathrm{g} / \mathrm{ml}$ (see Figures 16a-f).

The experimental results were in reasonable agreement with theory, which not only validated our model, but verified our experimental methods and illustrated the accuracy of our measurements. The general trends of the experimental data followed that of the theory (previously described). The only consistent difference was that the theoretical fluorescence was always slightly less than what was measured at the closer radial distances. This discrepancy worsened for the smaller drug concentrations as the thickness of the top layer was increased, but remained relatively consistent for the larger drug concentrations. 


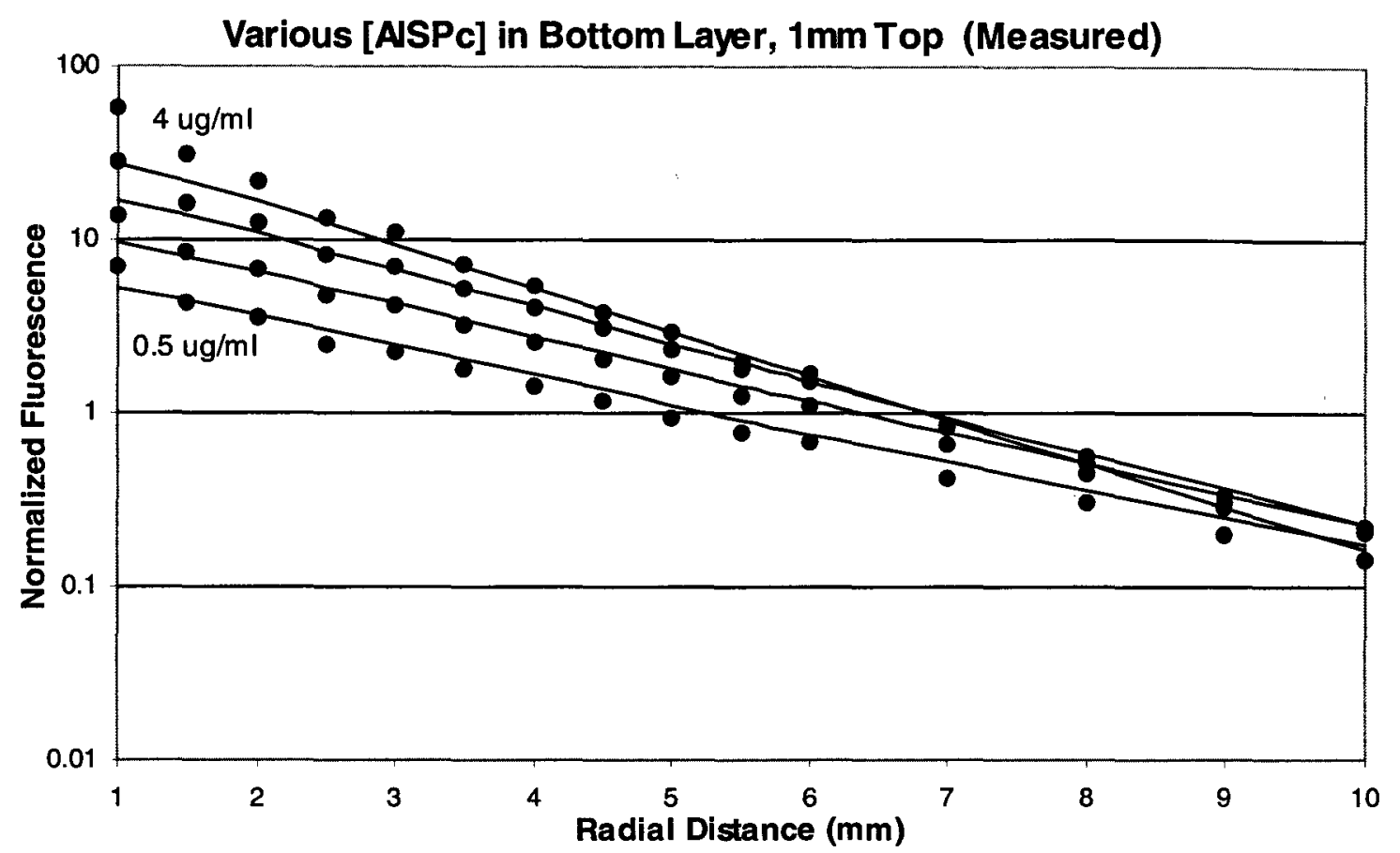

Figure 16a: Theory (lines) and Phantom Measurement (points) for $0.5,1,2 \& 4 \mu \mathrm{g} / \mathrm{ml}$

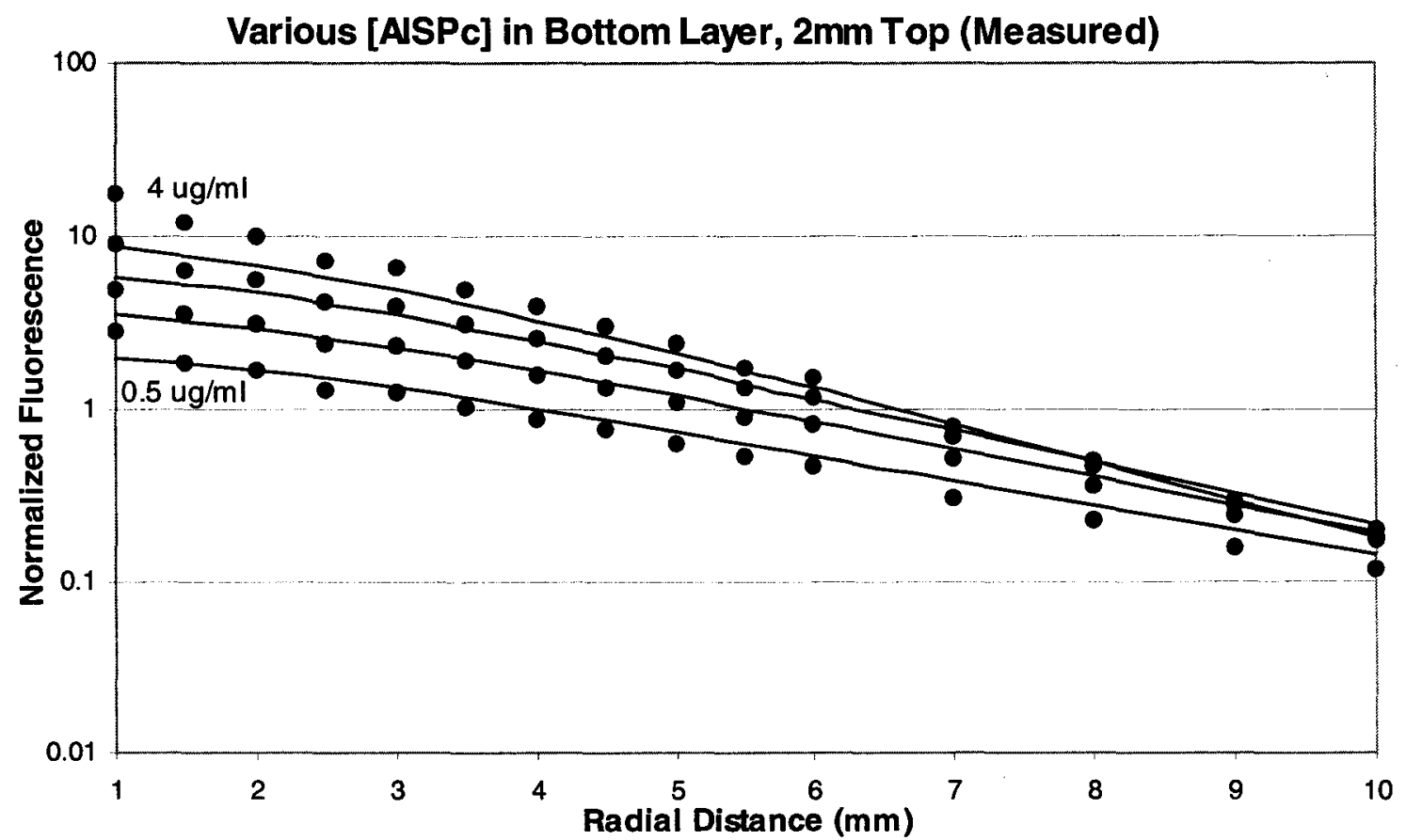

Figure 16b: Theory (lines) and Phantom Measurement (points) for $0.5,1,2 \& 4 \mu \mathrm{g} / \mathrm{ml}$ 


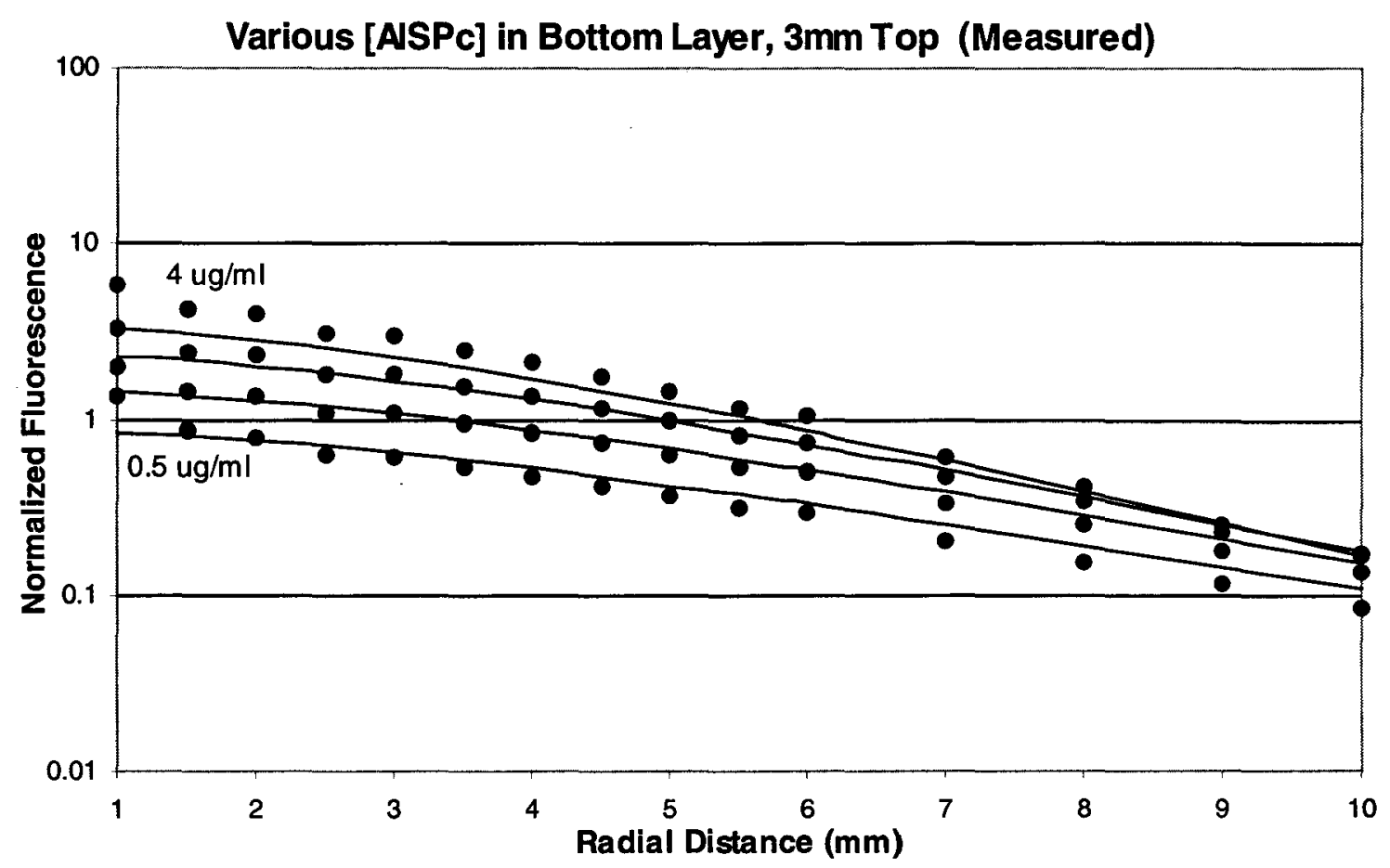

Figure 16c: Theory (lines) and Phantom Measurement (points) for 0.5, 1, $2 \& 4 \mu \mathrm{g} / \mathrm{ml}$

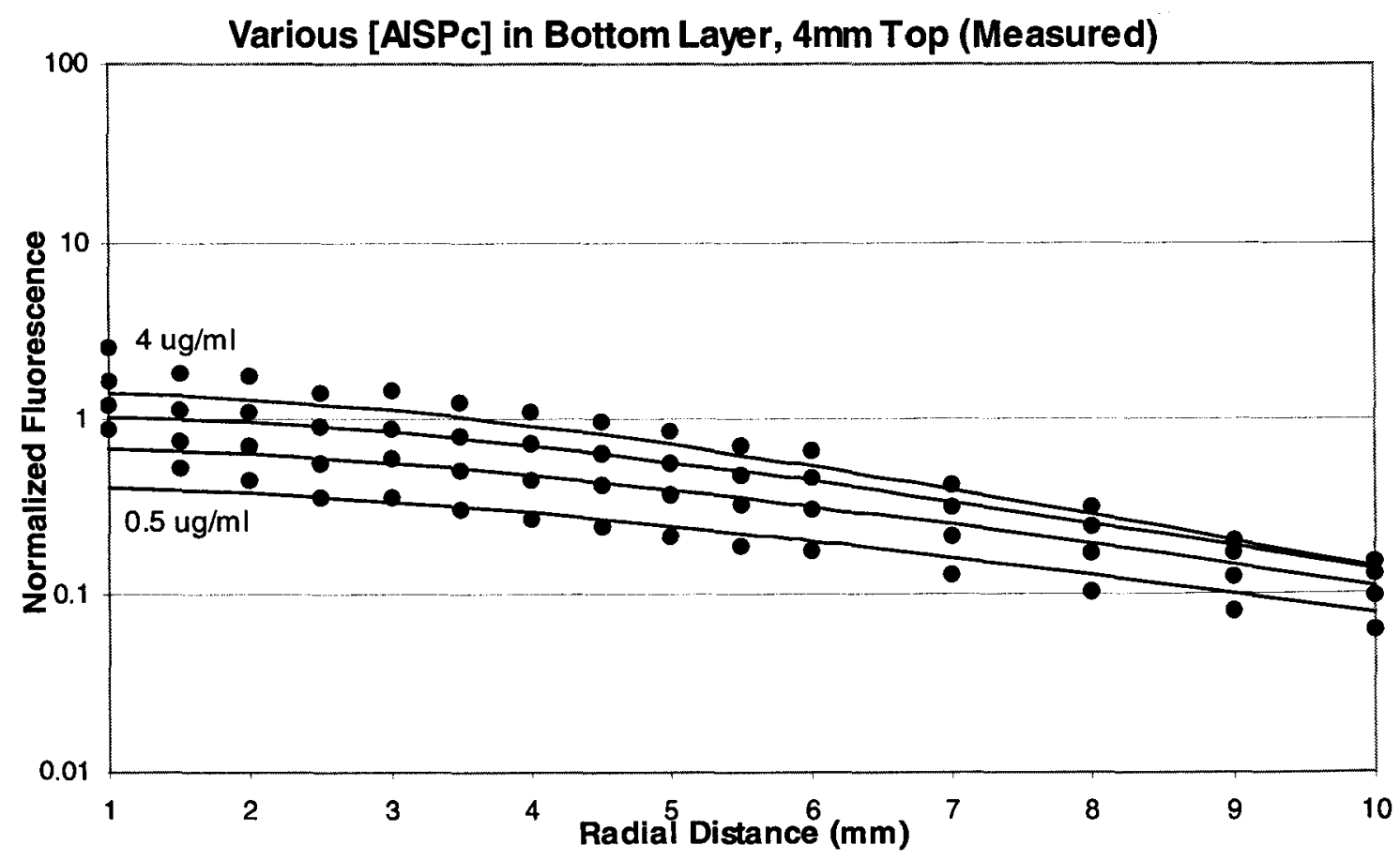

Figure 16d: Theory (lines) and Phantom Measurement (points) for 0.5, 1, $2 \& 4 \mu \mathrm{g} / \mathrm{ml}$ 


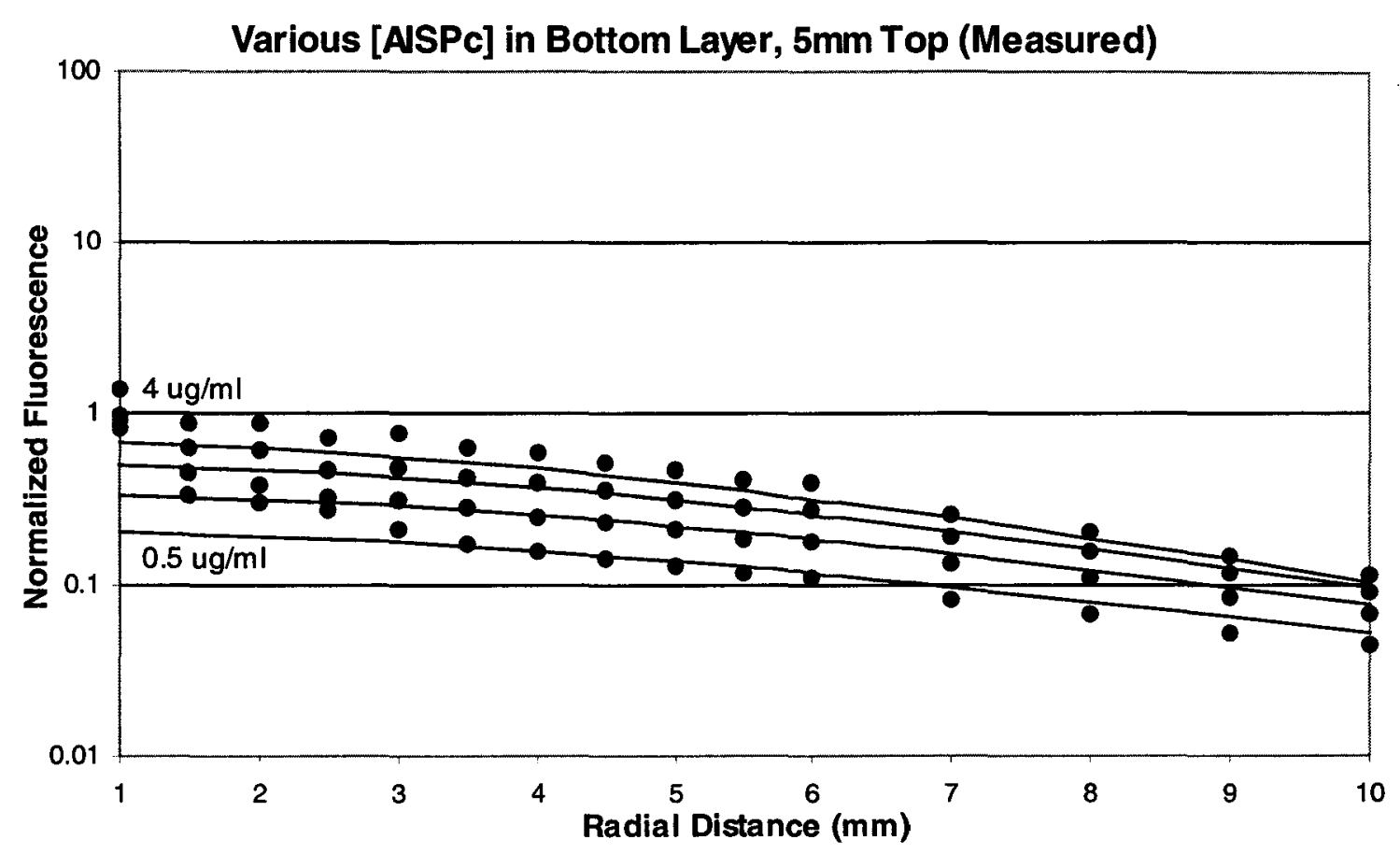

Figure 16e: Theory (lines) and Phantom Measurement (points) for $0.5,1,2 \& 4 \mu \mathrm{g} / \mathrm{ml}$

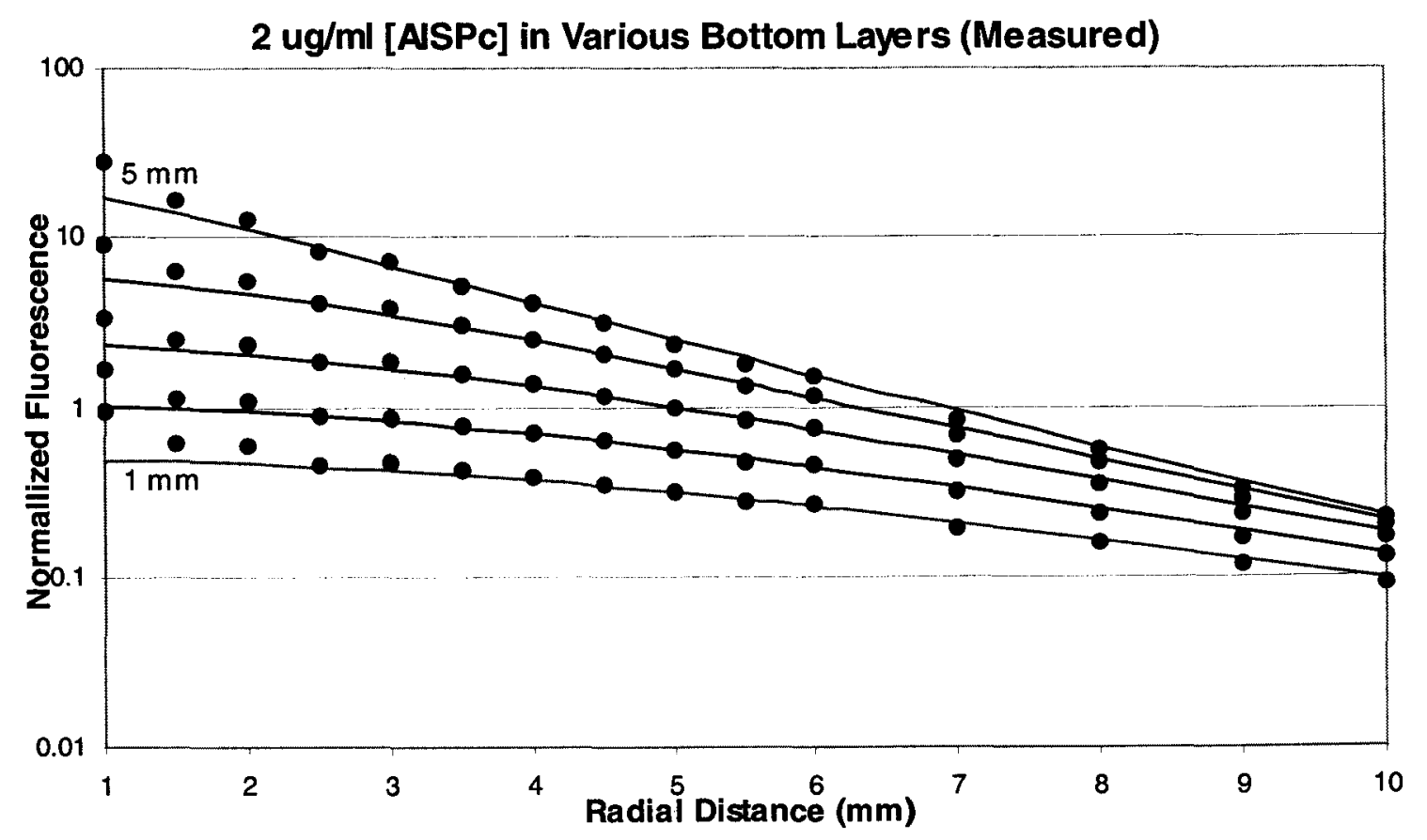

Figure 16f: Theory (lines) and Phantom Measurement (points) for $0.5,1,2 \& 4 \mu \mathrm{g} / \mathrm{ml}$ 


\subsection{Drug in Top Layer}

\section{Diffusion Theory and Monte Carlo}

This theoretical data was previously produced using another set of optical properties, such that $\mu_{\mathrm{s}}^{\prime}=1.0 \mathrm{~mm}^{-1}$ and $\mu_{\mathrm{a}}=0.01 \mathrm{~mm}^{-1}$ at both the excitation and fluorescent wavelengths. Top layer thicknesses, of 1 to $5 \mathrm{~mm}$, were analysed for clinically appropriate drug concentrations of $0.5,1,2,4$ and $6 \mu \mathrm{g} / \mathrm{ml}$ (see Figures 17a-e). Again, the Monte Carlo and Diffusion theory were normalized with respect to one photon, and the diffusion theory consistently produced a lower fluorescence intensity.

Once again, the drug concentrations were clearly discernible for each of the measured thicknesses. As the thickness of the top (drug-containing) layer was increased with the lower drug concentrations, the fluorescence intensity was increased at the larger radial distances. Therefore, as substantially more fluorescence was detected at the distant tracks, the slopes of the spatially resolved fluorescence curves decreased. Contrarily, as the thickness of the top layer was increased with the higher drug concentrations, the fluorescence intensity decreased in the distant tracks since more and more fluorescence was being reabsorbed. This caused the fluorescence curves from the higher concentrations to cross over that of the lower concentrations.

When comparing the spatially resolved fluorescence for a number of top layer thicknesses at one concentration (see Figure 17f), it was shown that as the thickness increased, it became more difficult to determine the thickness of the layer. 


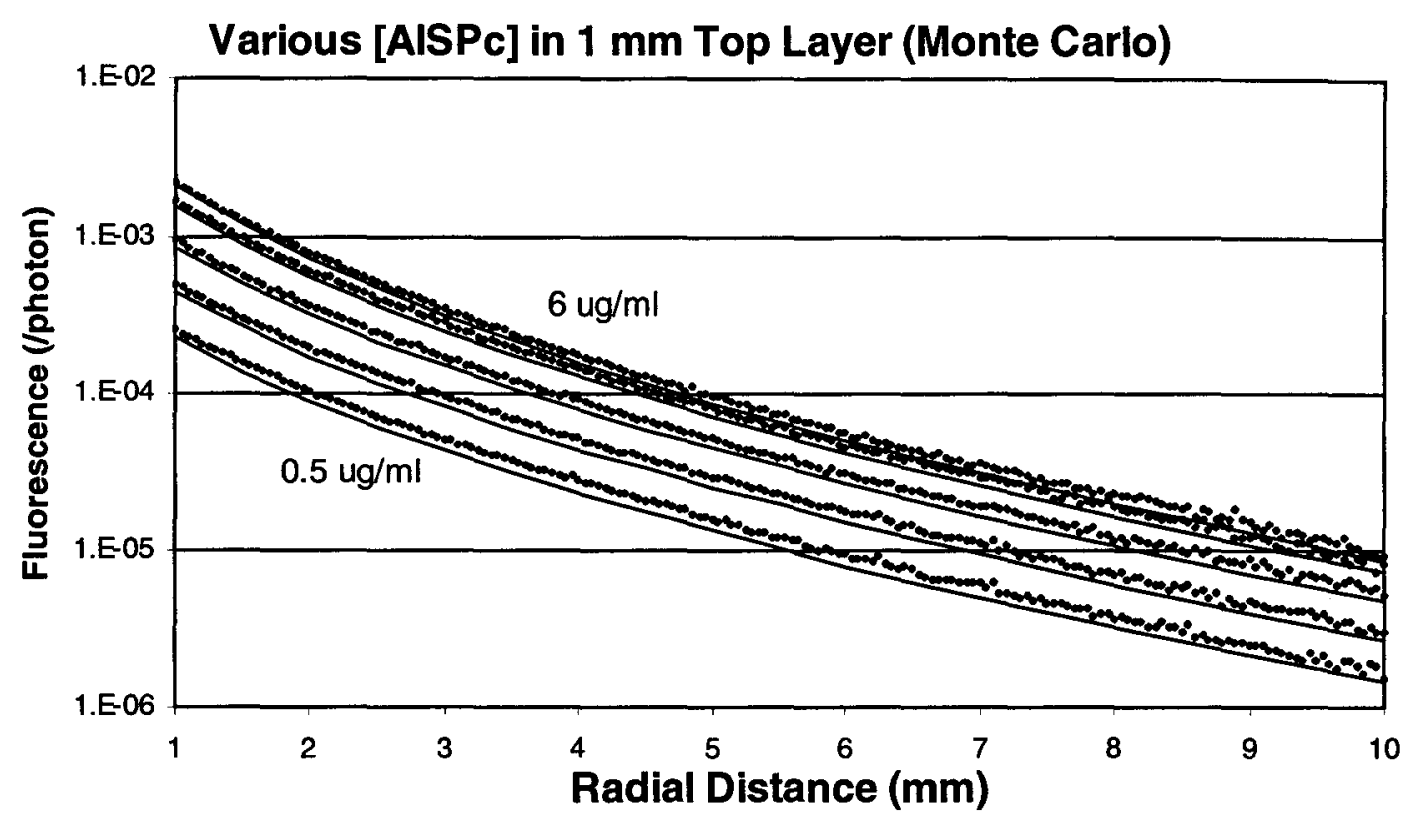

Figure 17a: Diffusion theory (lines) and Monte Carlo (points) for $0.5,1,2,4$ and $6 \mu \mathrm{g} / \mathrm{ml}$

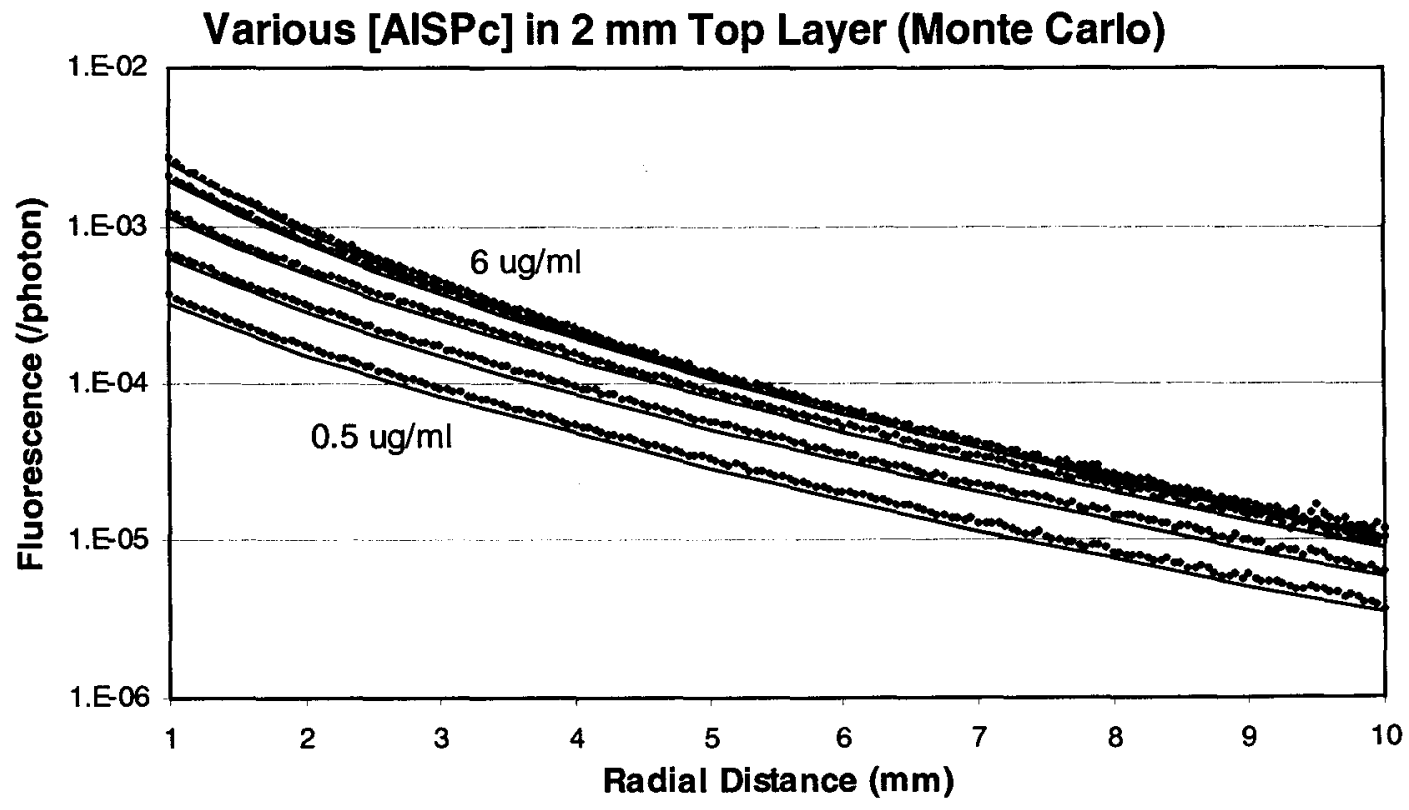

Figure 17b: Diffusion theory (lines) and Monte Carlo (points) for $0.5,1,2,4$ and $6 \mu \mathrm{g} / \mathrm{ml}$ 


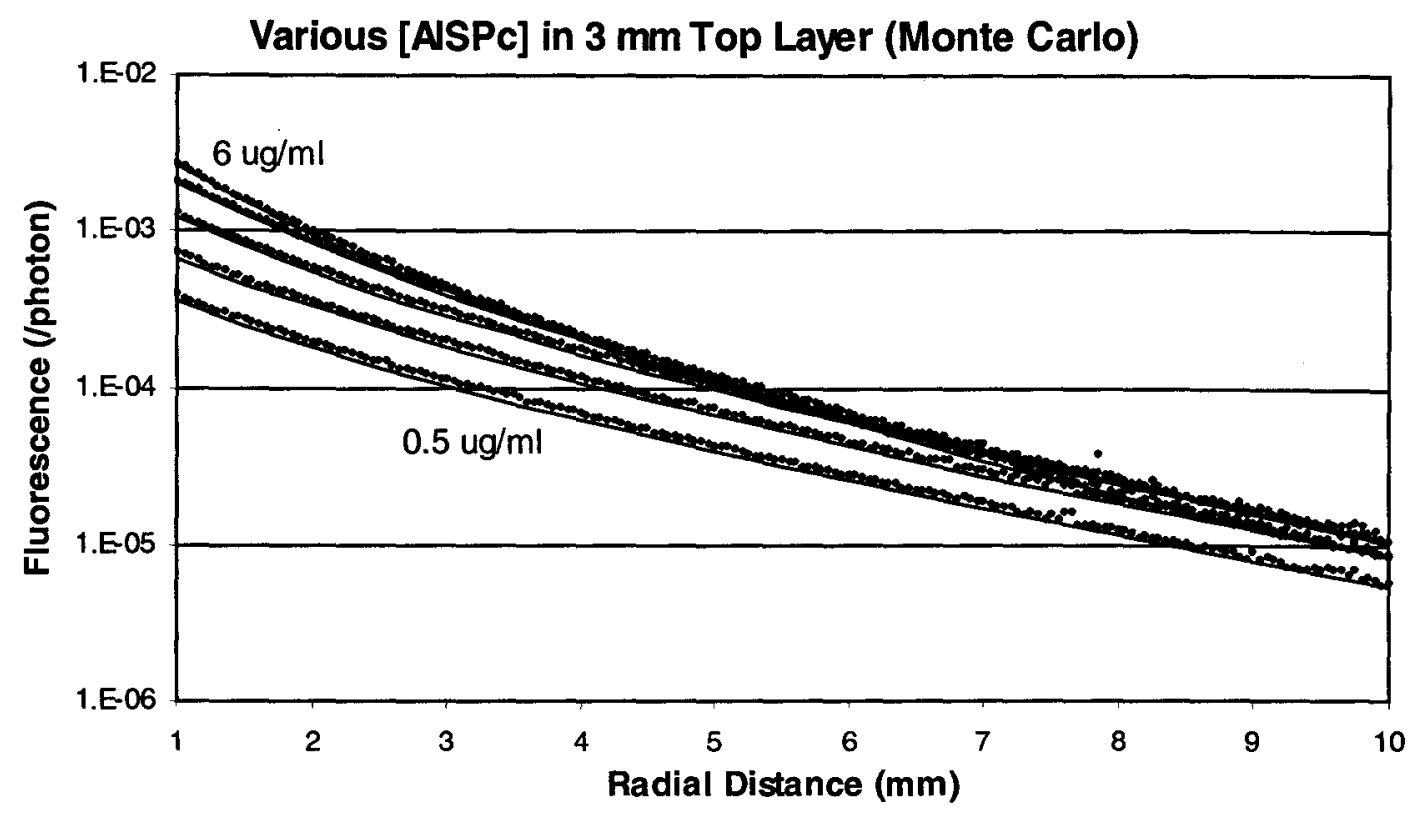

Figure 17c: Diffusion theory (lines) and Monte Carlo (points) for $0.5,1,2,4$ and $6 \mu \mathrm{g} / \mathrm{ml}$

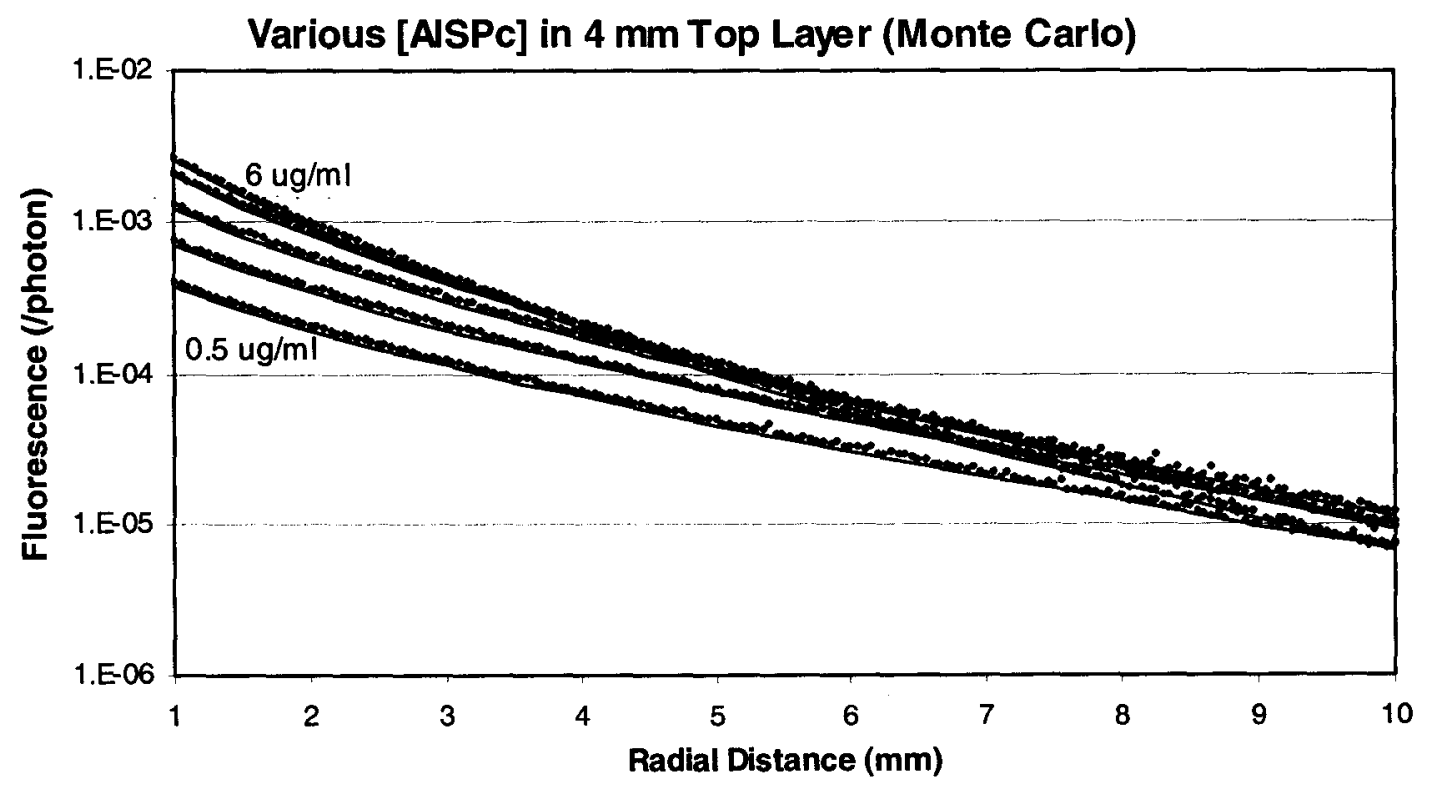

Figure 17d: Diffusion theory (lines) and Monte Carlo (points) for $0.5,1,2,4$ and $6 \mu \mathrm{g} / \mathrm{ml}$ 


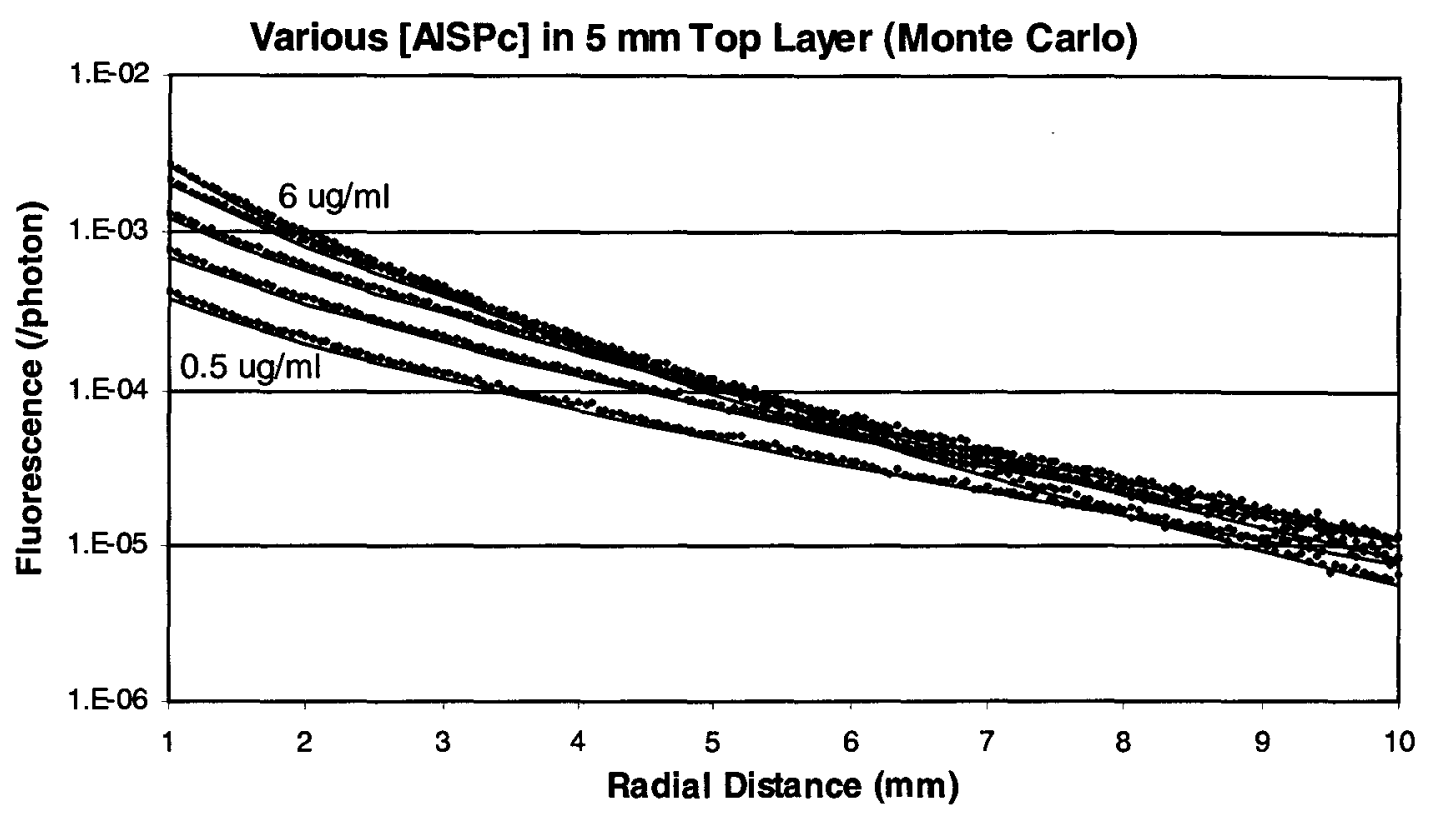

Figure 17e: Diffusion theory (lines) and Monte Carlo (points) for $0.5,1,2,4$ and $6 \mu \mathrm{g} / \mathrm{ml}$

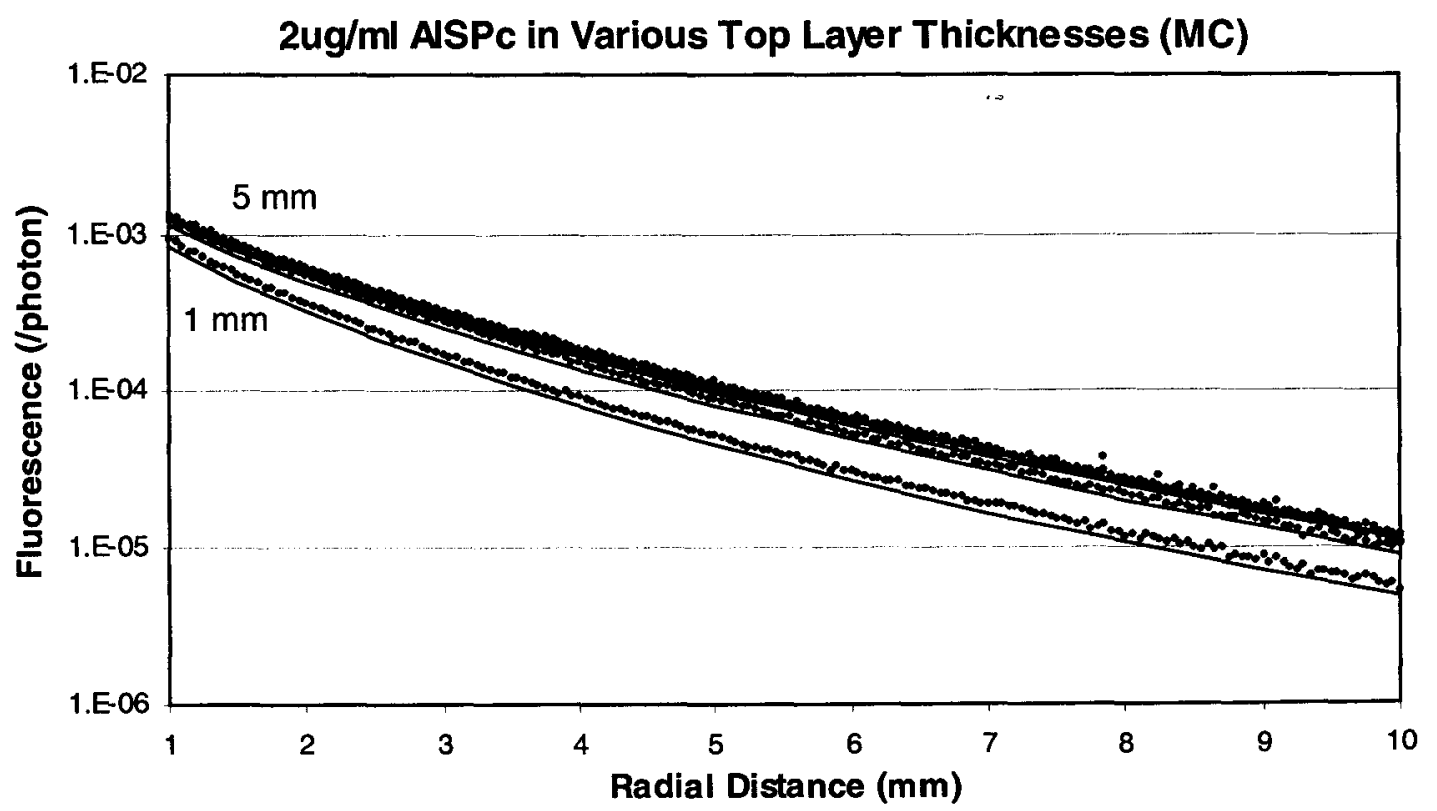

Figure 17f: Diffusion theory (lines) and Monte Carlo (points) for $0.5,1,2,4$ and $6 \mu \mathrm{g} / \mathrm{ml}$ 
Consequently, the ability to discern the thickness of the layer appears to be limited to a maximum top layer thickness of 4 to $5 \mathrm{~mm}$.

\section{Diffusion Theory and Experimental Measurements}

The experimental optical properties were used in this comparison ie. $\mu_{\mathrm{s}}^{\prime}(@ 632 \mathrm{~nm})=$ $1.44 \mathrm{~mm}^{-1}, \mu_{\mathrm{s}}^{\prime}(@ 683 \mathrm{~nm})=1.14 \mathrm{~mm}^{-1}$, and $\mu_{\mathrm{a}}=0.013 \mathrm{~mm}^{-1}$ for both wavelengths. Again the fluorescence from an $\left[\mathrm{AlS}_{4} \mathrm{Pc}\right]$ of $2 \mu \mathrm{g} / \mathrm{ml}, 3 \mathrm{~mm}$ top layer thickness, at a radial distance of $5 \mathrm{~mm}$ was used to normalize the experimental measurements and theory.

The slopes and intensities of the fluorescence measurements match that of the theory, with the exception of the intensities for the $1 \mathrm{~mm}$ top layer thickness (see Figure 18a-f). Since the intensity of the theoretical data was slightly higher, it was thought that the probe may have been suspended slightly higher than the surface of the plastic sheet such that the reference point was not actually at a depth of $0 \mathrm{~mm}$. Although the difference between each layer thickness was measured to $1 \mathrm{~mm}$, the reference point may have been shifted by as much as $1 \mathrm{~mm}$ (as observed while using water alone), due to differential fluid pressures. Consequently, the theoretical thicknesses were increased, and after the addition of $0.6 \mathrm{~mm}$ the theory corresponded to the measured data, and there were no significant discrepancies (see Figures 19a-f). 


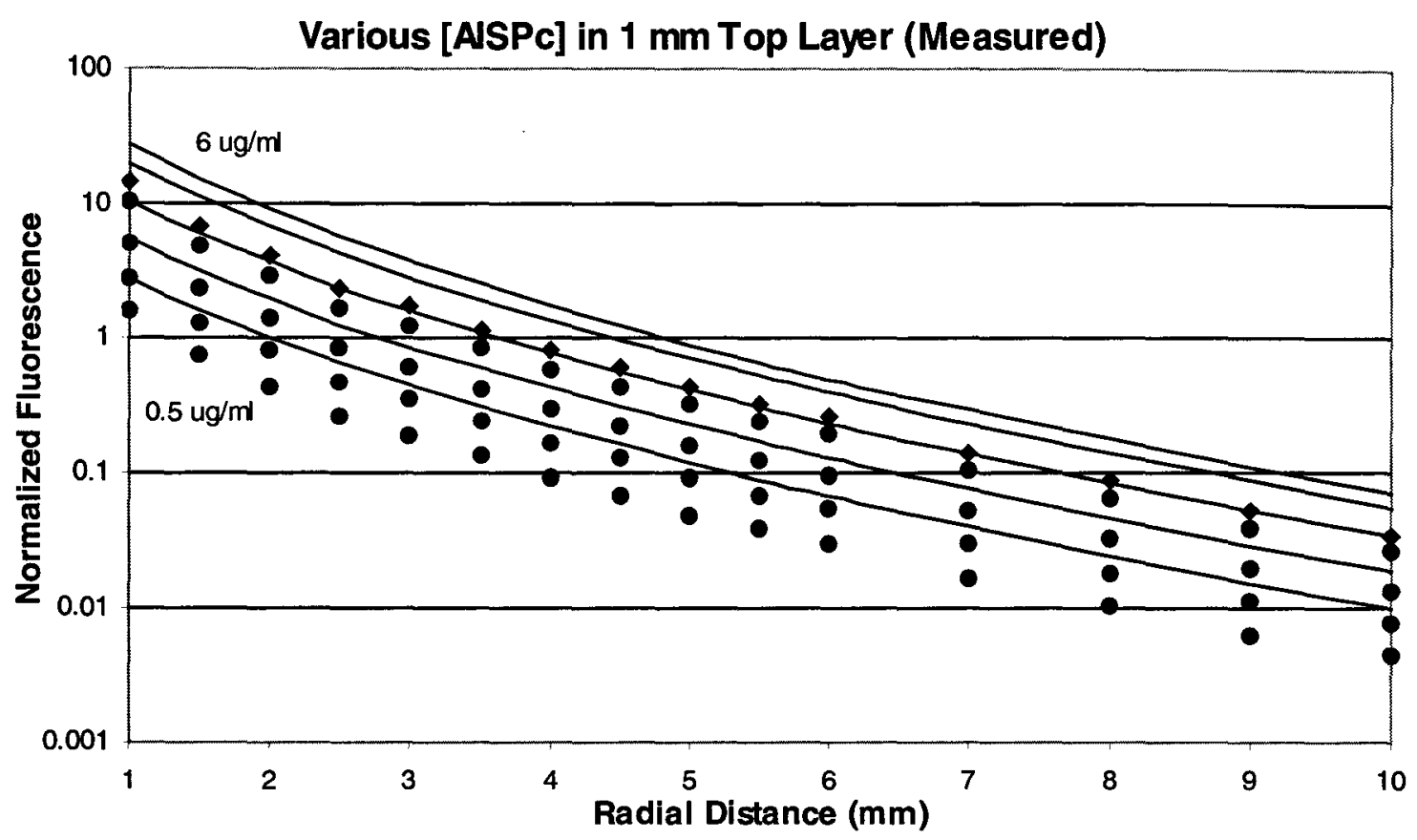

Figure 18a: Theory (lines) and Phantom Measurements (points) for $0.5,1,2,4 \& 6 \mu \mathrm{g} / \mathrm{ml}$

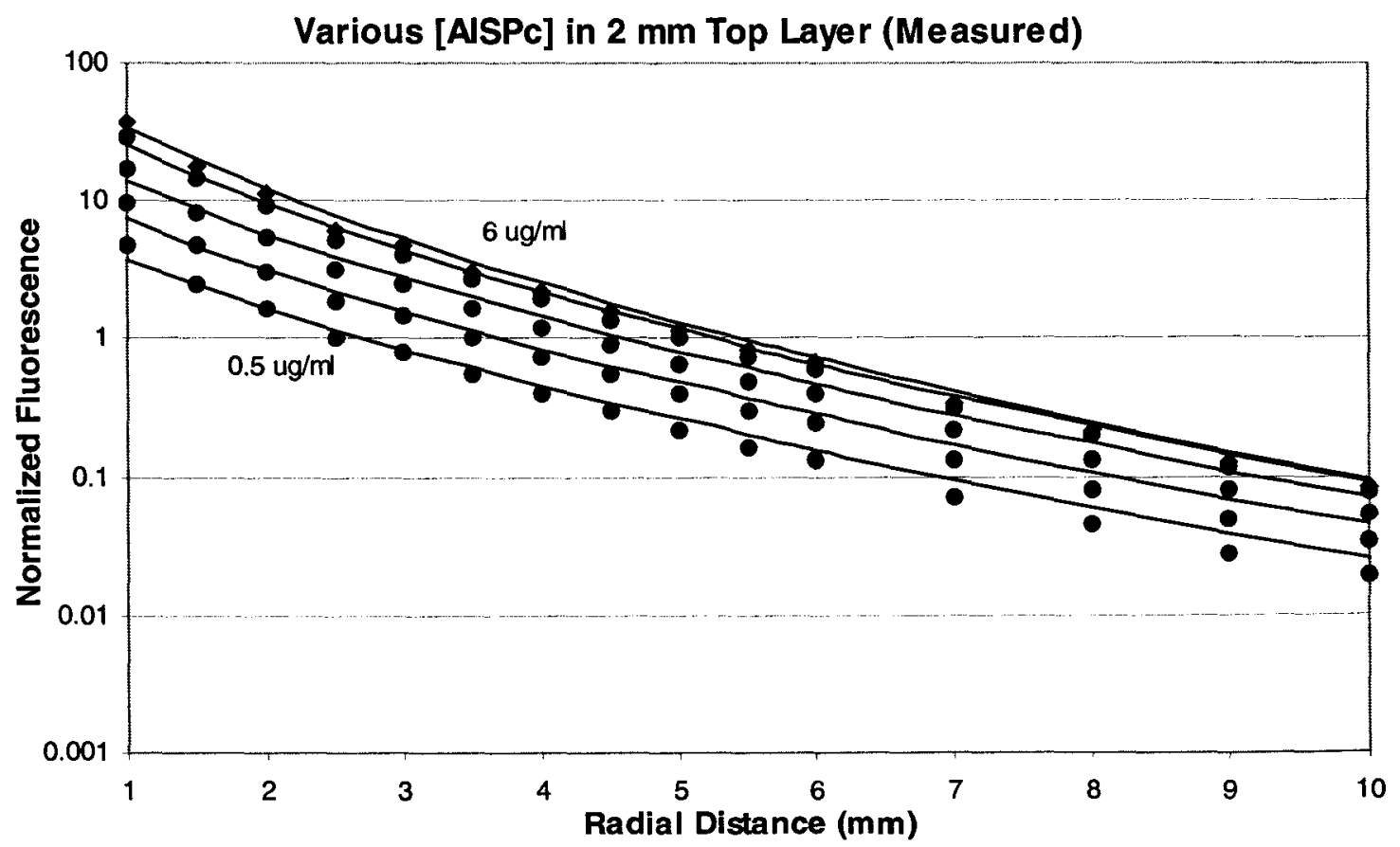

Figure 18b: Theory (lines) and Phantom Measurements (points) for 0.5, 1, 2, $4 \& 6 \mu \mathrm{g} / \mathrm{ml}$ 


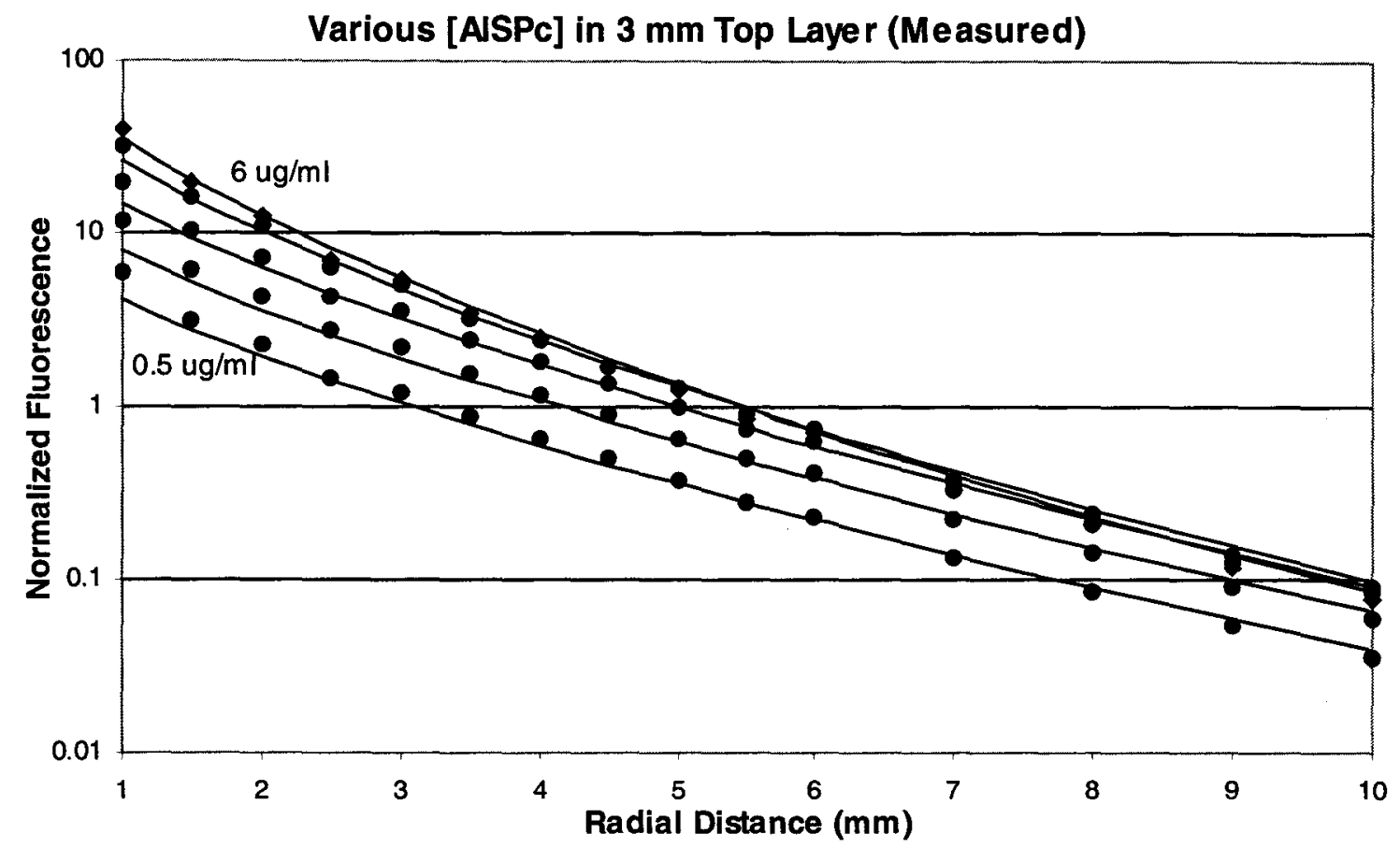

Figure 18c: Theory (lines) and Phantom Measurements (points) for $0.5,1,2,4 \& 6 \mu \mathrm{g} / \mathrm{ml}$

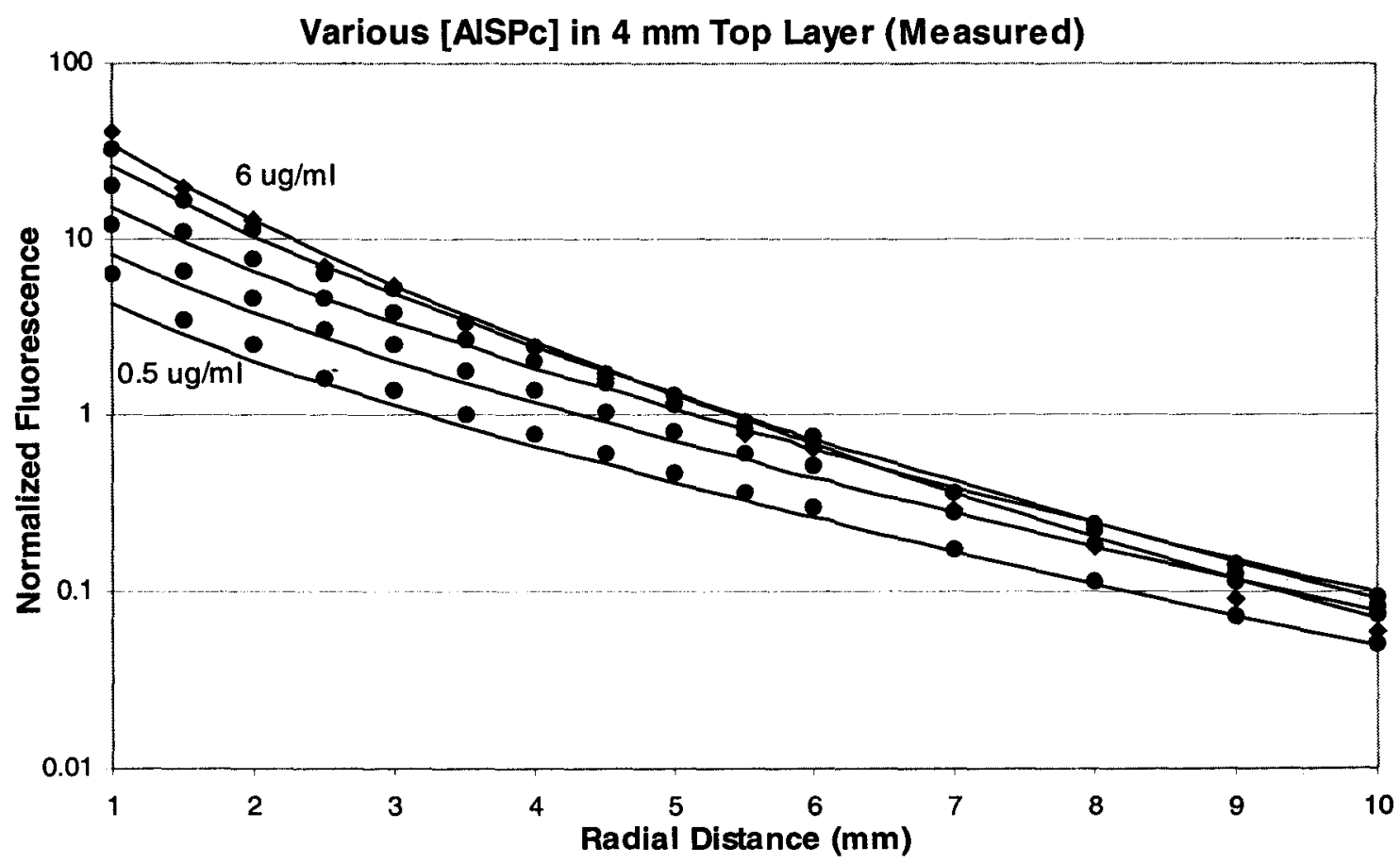

Figure 18d: Theory (lines) and Phantom Measurements (points) for $0.5,1,2,4 \& 6 \mu \mathrm{g} / \mathrm{ml}$ 


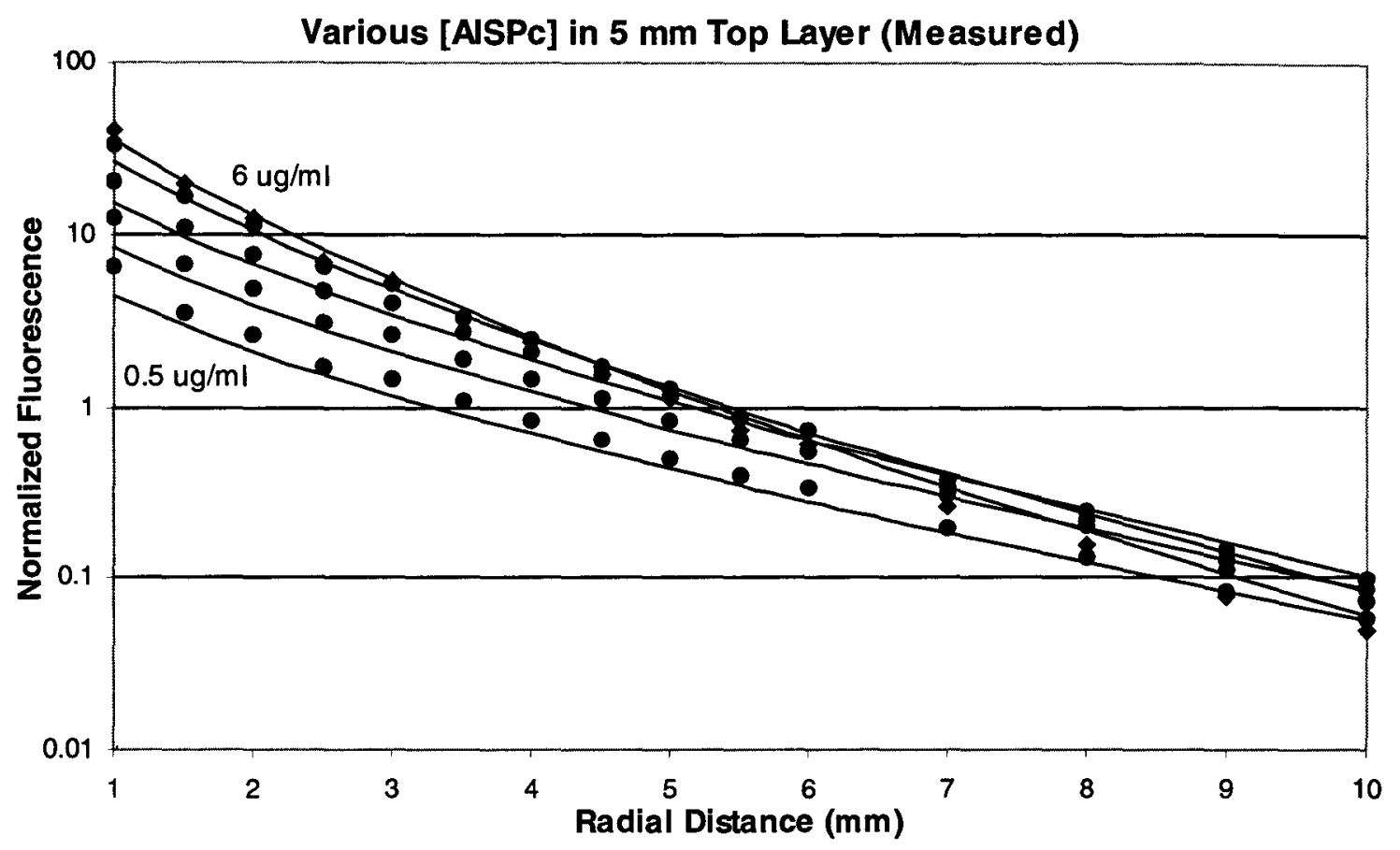

Figure 18e: Theory (lines) and Phantom Measurements (points) for $0.5,1,2,4 \& 6 \mu \mathrm{g} / \mathrm{ml}$

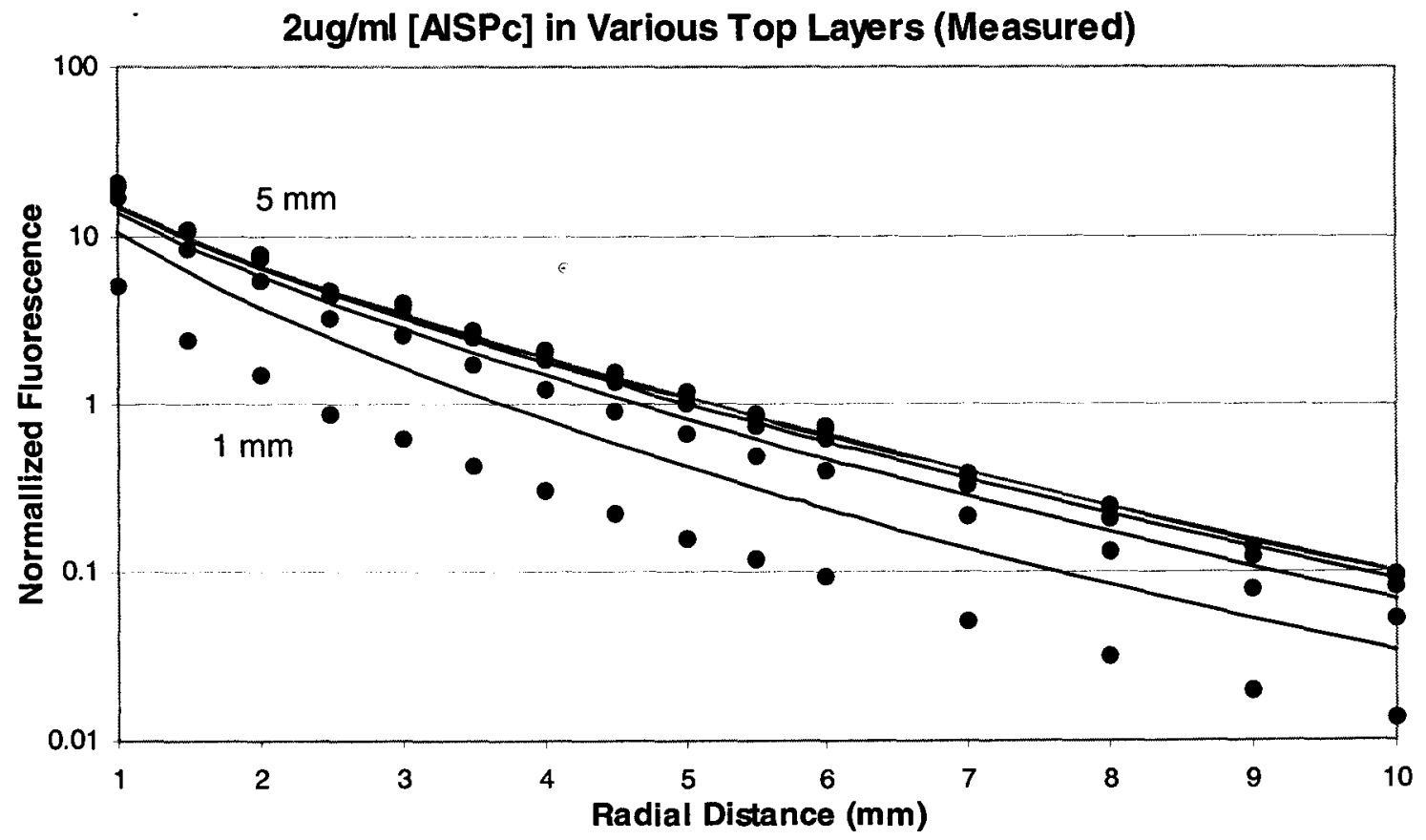

Figure 18f: Theory (lines) and Phantom Measurements (points) for $0.5,1,2,4 \& 6 \mu \mathrm{g} / \mathrm{ml}$ 


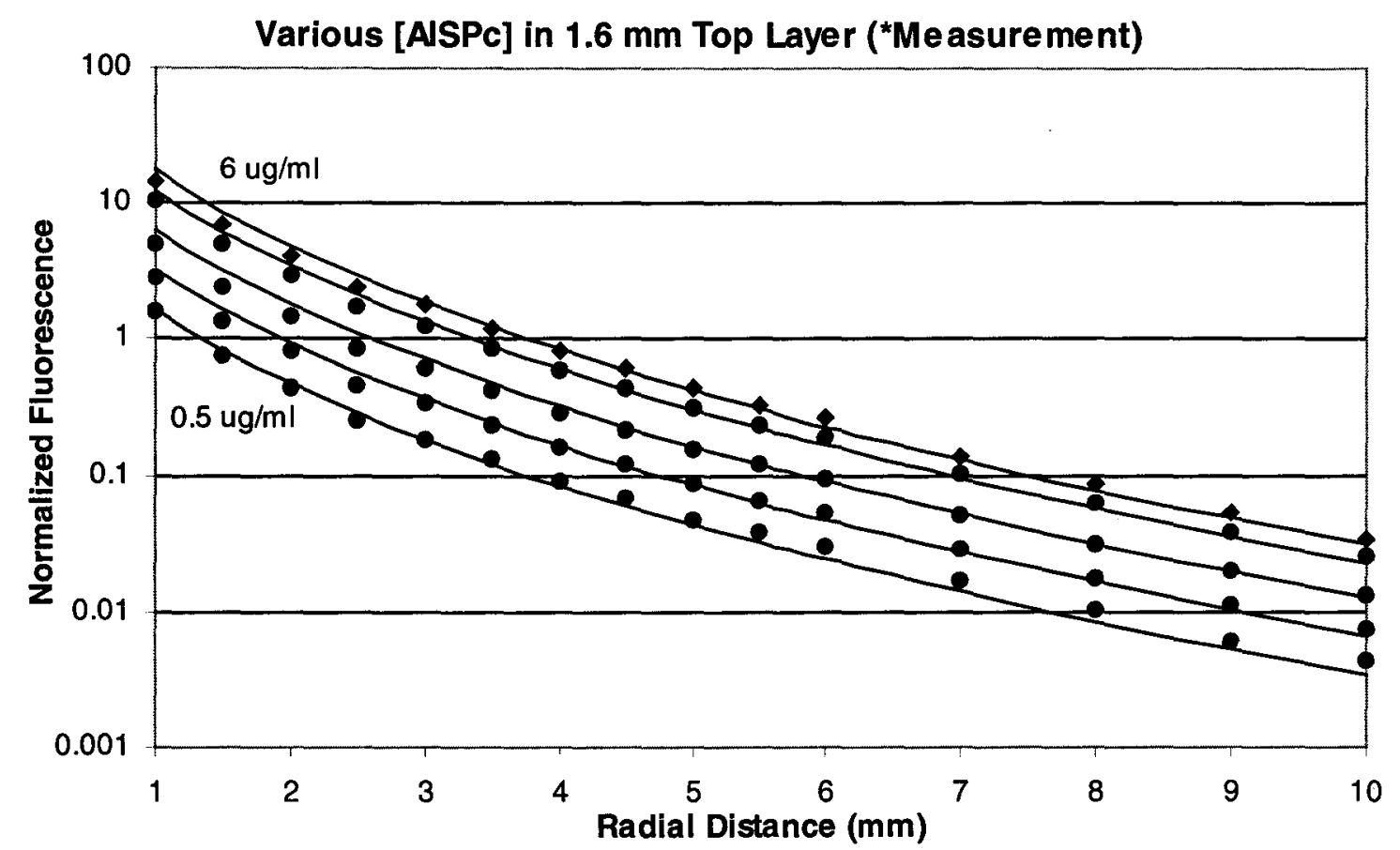

Figure 19a: Adjusted Theory (lines) and Measurements (points) for $0.5,1,2,4 \& 6 \mu \mathrm{g} / \mathrm{ml}$

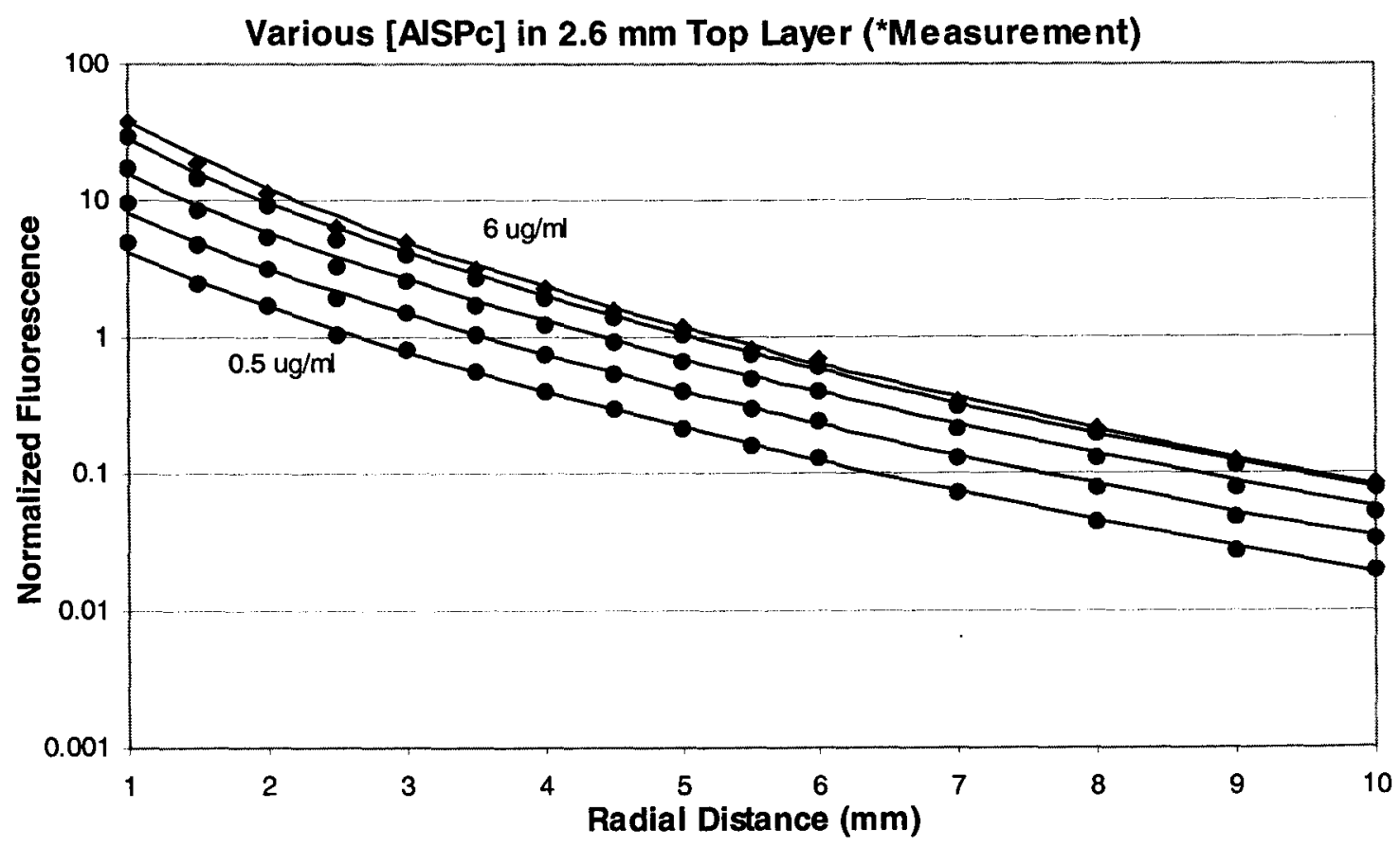

Figure 19b: Adjusted Theory (lines) and Measurements (points) for $0.5,1,2,4 \& 6 \mu \mathrm{g} / \mathrm{ml}$ 


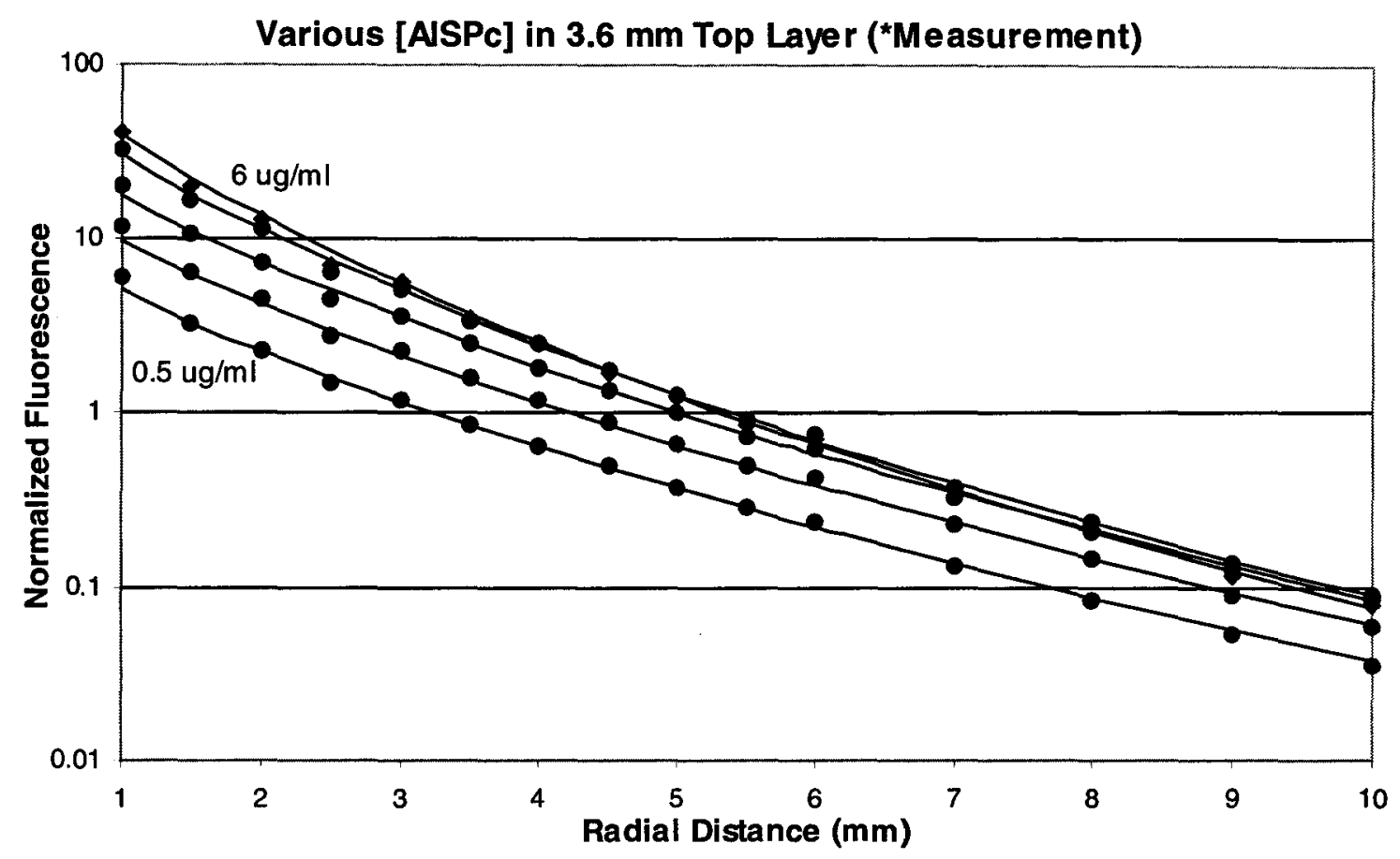

Figure 19c: Adjusted Theory (lines) and Measurements (points) for $0.5,1,2,4 \& 6 \mu \mathrm{g} / \mathrm{ml}$

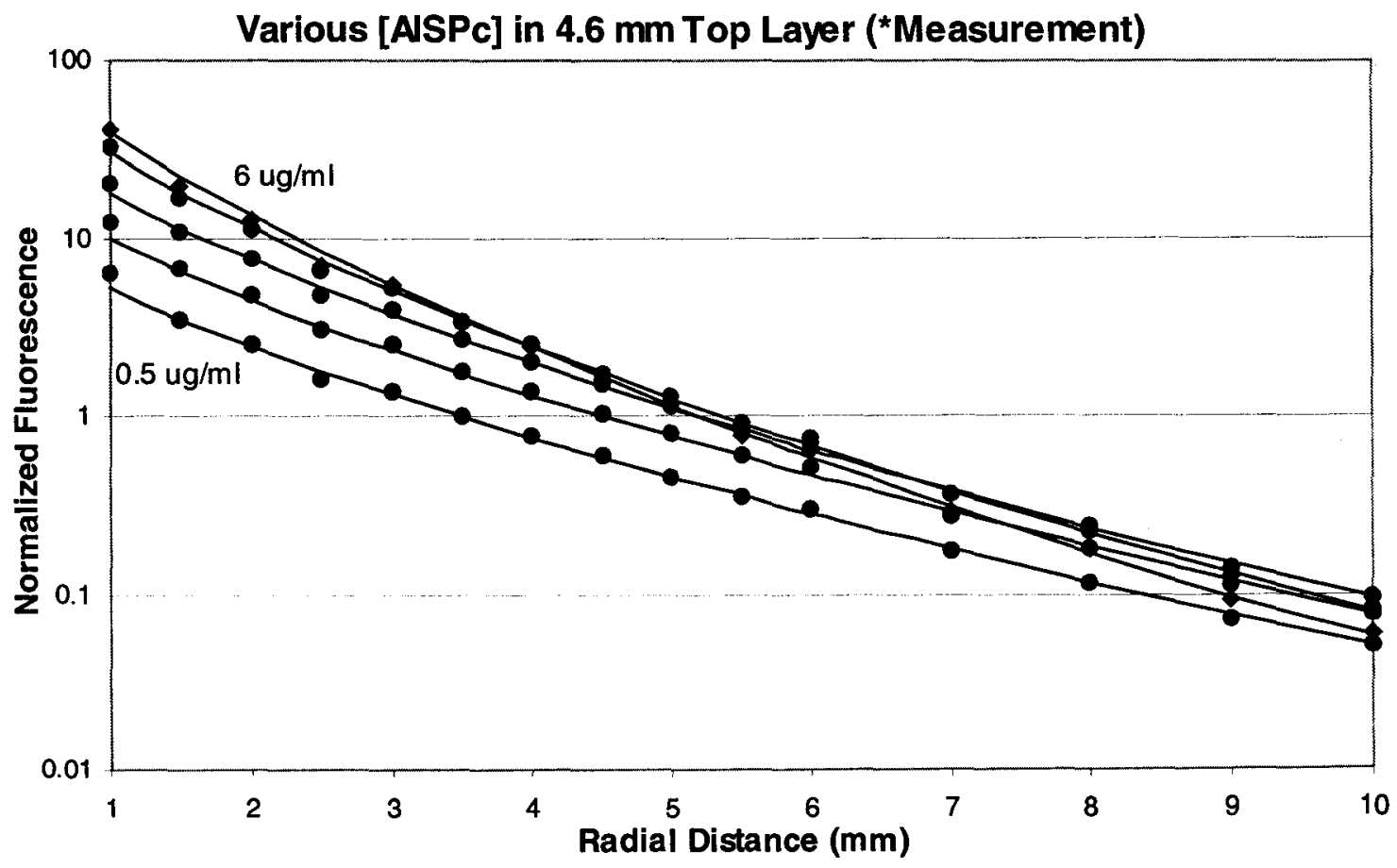

Figure 19d: Adjusted Theory (lines) and Measurements (points) for 0.5, 1, 2, $4 \& 6 \mu \mathrm{g} / \mathrm{ml}$ 


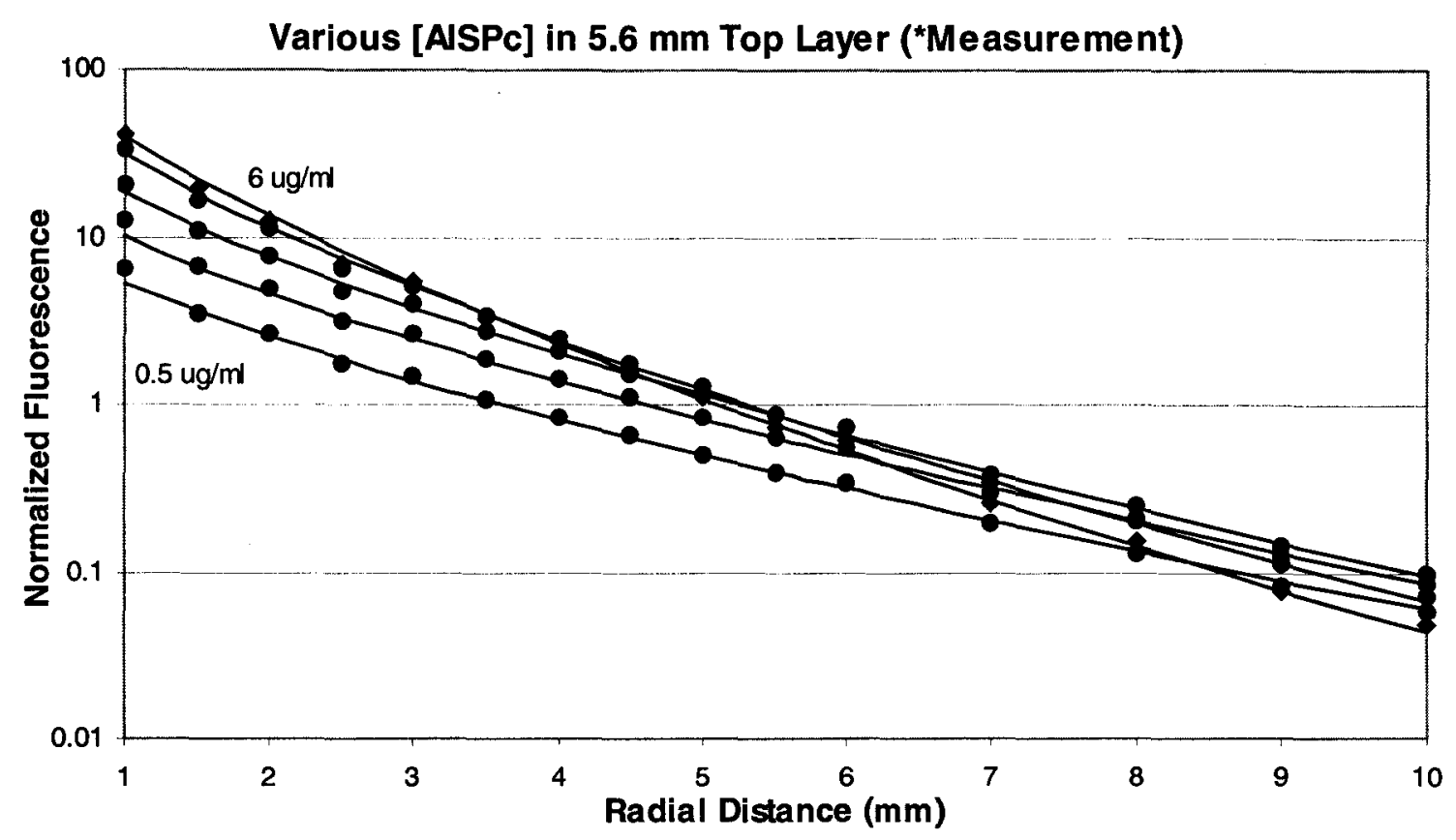

Figure 19e: Adjusted Theory (lines) and Measurements (points) for 0.5, 1, 2, $4 \& 6 \mu \mathrm{g} / \mathrm{ml}$

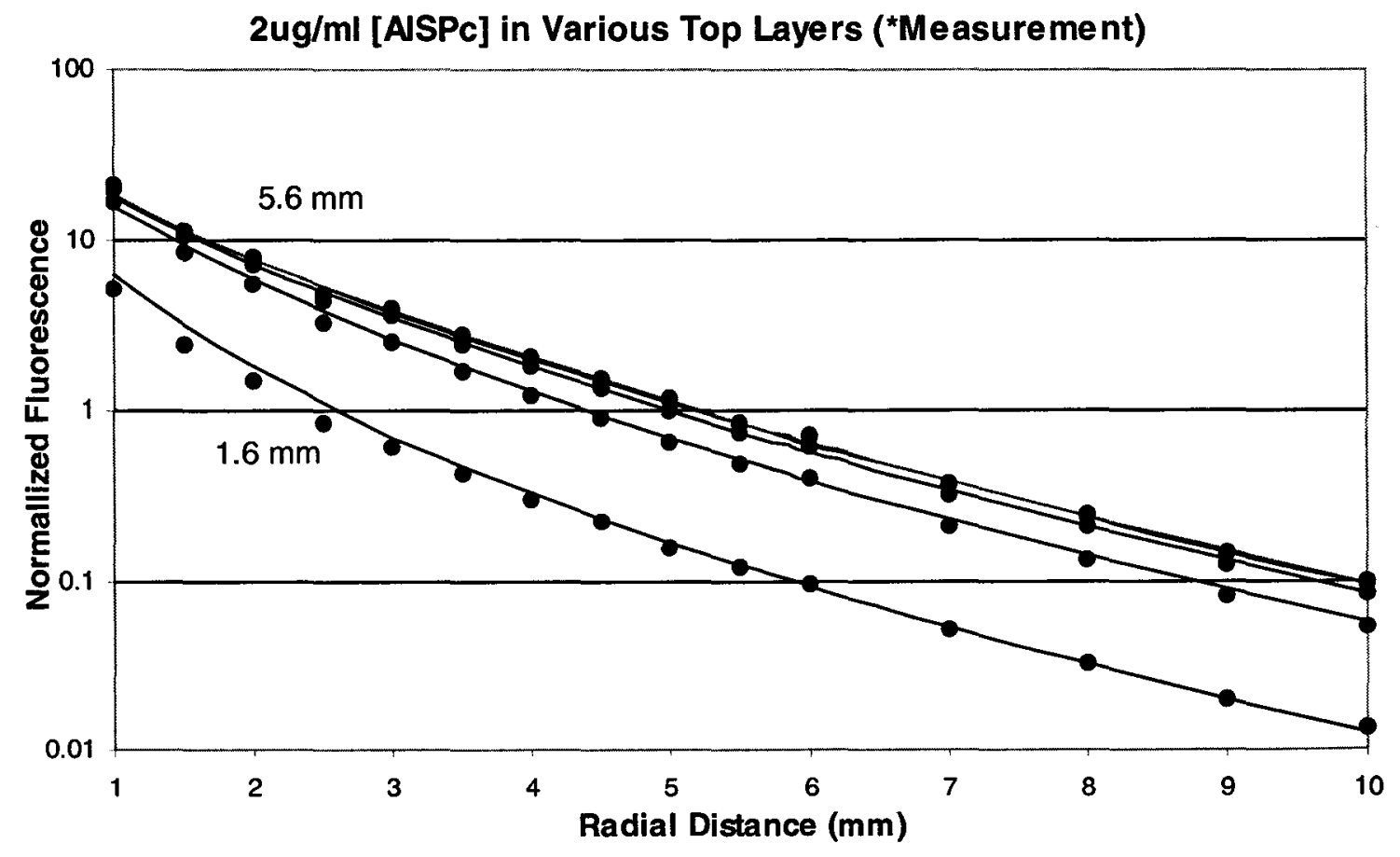

Figure 19f: Adjusted Theory (lines) and Measurements (points) for $0.5,1,2,4 \& 6 \mu \mathrm{g} / \mathrm{ml}$ 


\subsection{Variable Drug Distributions (Theory and Monte Carlo)}

\section{Simple Exponential Distribution (Similar to Drug Uptake)}

Simple exponential drug distributions $\left(\mathrm{e}^{-0.1 \mathrm{x}}, \mathrm{e}^{-0.5 \mathrm{x}}\right.$ and $\left.\mathrm{e}^{-\mathrm{x}}\right)$ were utilized in our model to evaluate the use of spatially resolved fluorescence measurements to quantify drug diffusion into skin (Figures 20a \& b). The fluorescence intensities from the model were slightly lower than the Monte Carlo data, as expected, because at this time the residual contribution of the integral was not taken into account. Once normalized to the central point of each data set (fluorescence of $\mathrm{e}^{-0.5 \mathrm{x}}$ at a radial distance of $5 \mathrm{~mm}$ ), it was clear that the shapes of the curves were similar. It should also be noted that each curve is distinct, indicating that a measurement of the spatially resolved fluorescence should provide information to quantify the uptake of a topically administered photosensitizer.

\section{Sigmoidal Distribution (Similar to Drug Bleaching)}

During the broad beam irradiation of a homogeneous drug concentration, the drug is bleached downward from the surface. This produces a sigmoidal distribution which progresses into the medium as the irradiation continues. Such distributions $\left(\left[1+\mathrm{e}^{-4(\mathrm{x}-1)}\right]^{-1}\right.$, $\left[1+e^{-4(x-3)}\right]^{-1}$, and $\left.\left[1+e^{-4(x-5)}\right]^{-1}\right)$ were used in our model to produce spatially resolved fluorescence intensities similar to what one would expect during PDT (Figures 21a \& b). The intensities and shapes of these curves were clearly unique, indicating that it may be possible to monitor the bleaching depth during treatment. For these distributions, the correlation of the diffusion theory and Monte Carlo data was highly improved. This may be due to the fact that there is only a small amount of drug near the surface. 


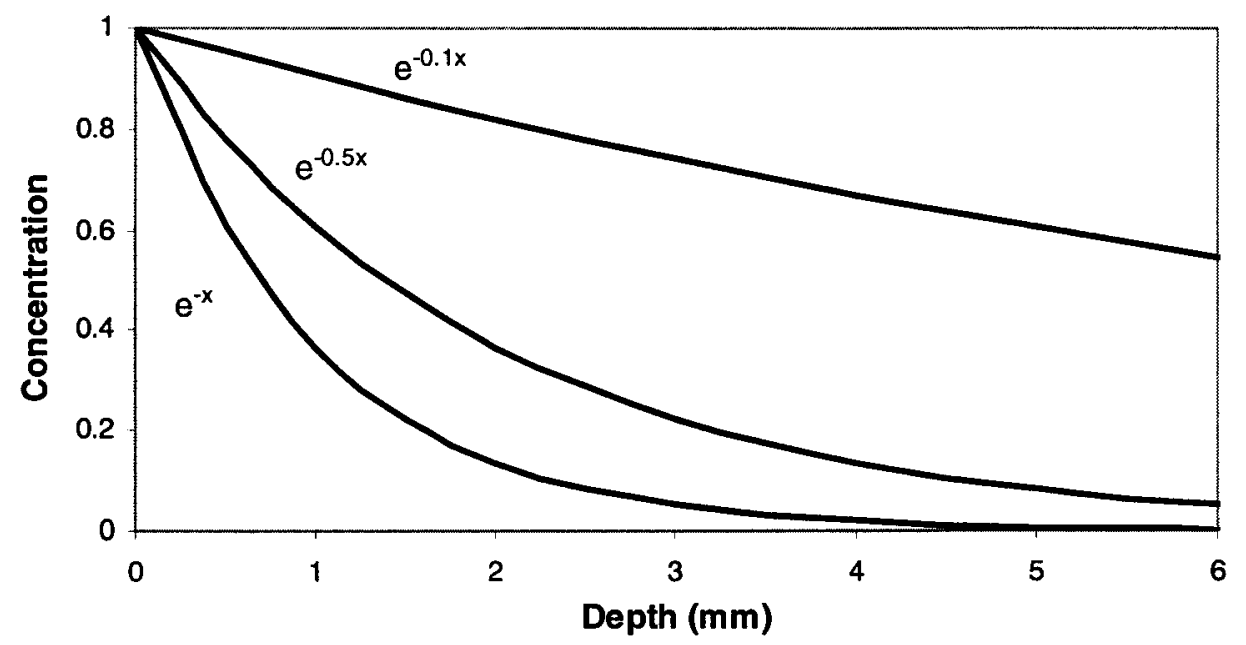

Figure 20a: Simple exponential drug distribution (similar to topical uptake)

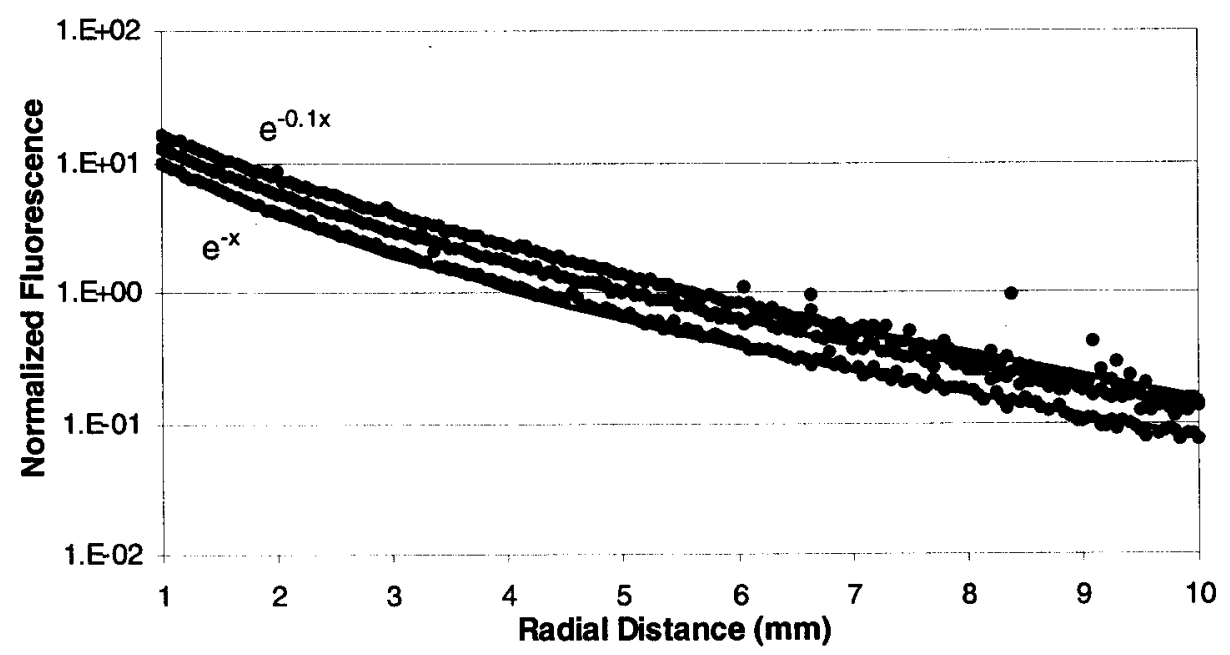

Figure 20b: Diffusion theory (lines) and Monte Carlo (points) for the spatially resolved fluorescence of the above drug distributions (optical properties similar to $\mathrm{AlS}_{4} \mathrm{Pc}$ in tissue). 


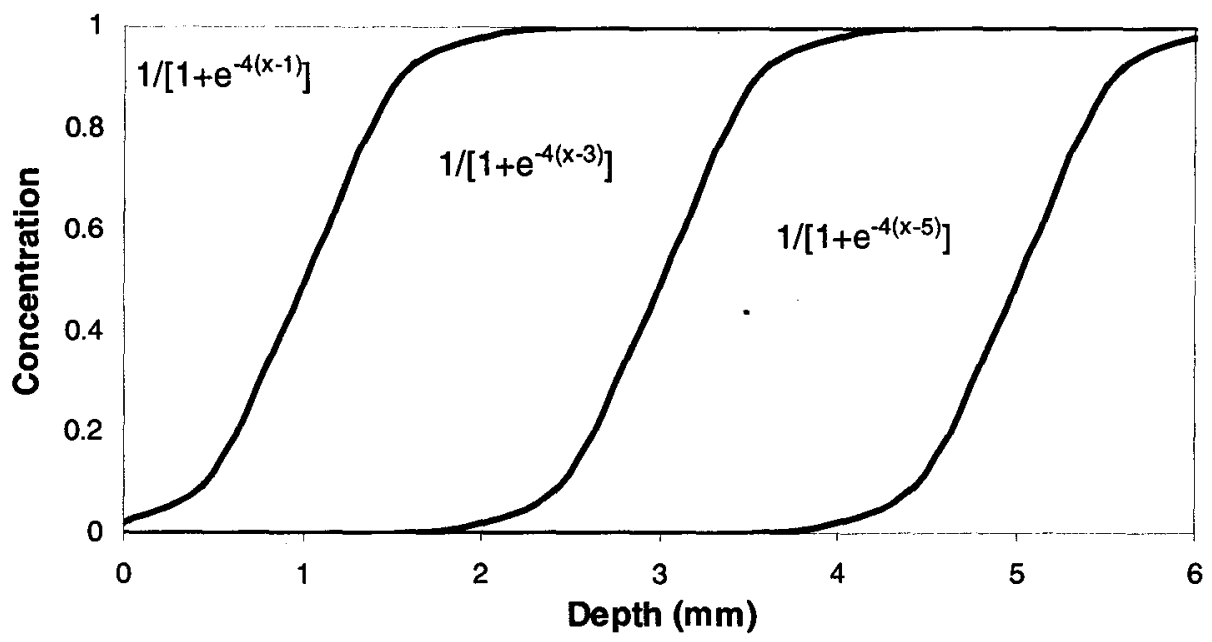

Figure 21a: Sigmoidal drug distributions (similar to instantaneous concentrations during photobleaching).

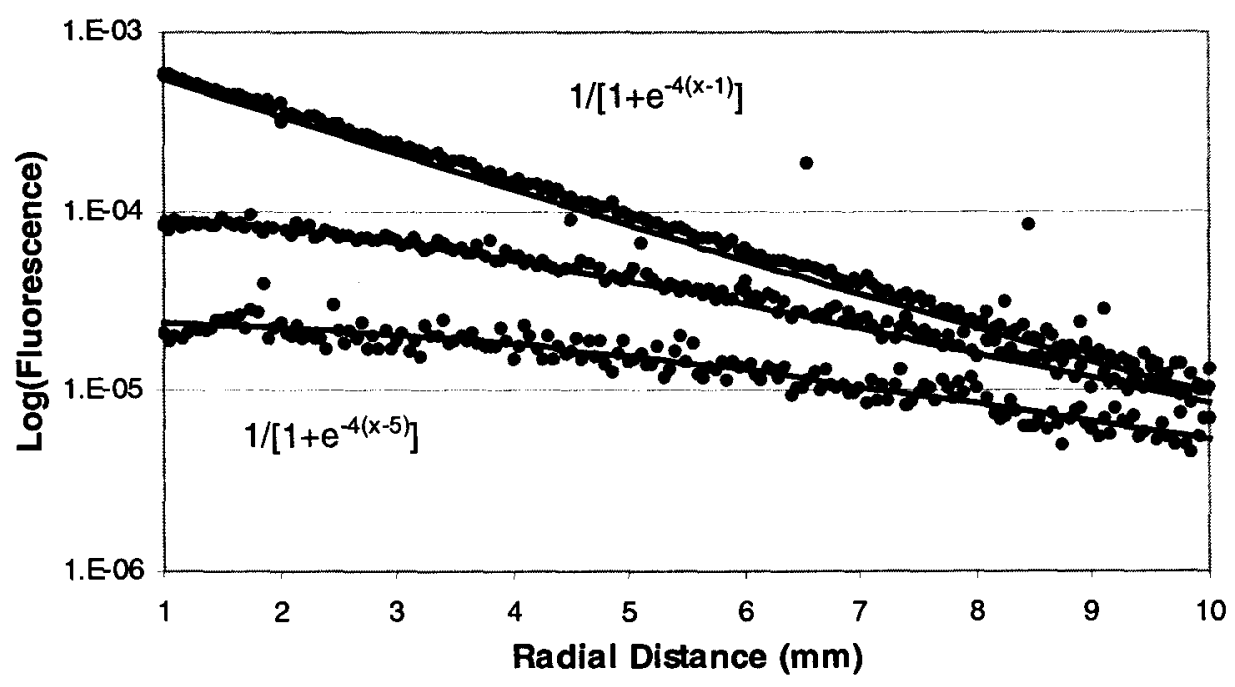

Figure 21b: Diffusion theory (lines) and Monte Carlo (points) for the spatially resolved fluorescence of the above drug distributions (optical properties similar to $\mathrm{AlS}_{4} \mathrm{Pc}$ in tissue). 


\section{DISCUSSION}

\subsection{Validity of Model}

As previously noted, the diffusion theory consistently produced a slightly lower fluorescence intensity than the Monte Carlo. It was originally thought that this was due to the fact that the model is not valid at distances which are close to the source, which is where the majority of the drug is found when the drug is contained in the top layer. Consequently, further comparisons to Monte Carlo data and experimental measurements are necessary to determine the source of this inconsistency.

Although the fluorescence magnitude produced by the model may not be accurate, the shapes of the spatially resolved fluorescence curves are generally correct. These shapes were examined for a range of clinically applicable drug concentrations and distributions, using appropriate optical properties for a common photosensitizer and tissue. It should also be noted that the absolute fluorescence magnitude may not be useful anyway, since it is difficult to accurately measure clinical fluences. Consequently, this suggests that it should be possible to use non-invasive measurements of the spatially resolved fluorescence to monitor the uptake of a topically administered photosensitizer, as well as its bleaching during treatment. 


\subsection{Modeling the Uptake of a Topically Applied Photosensitizer}

The topical administration of a photosensitizer was modeled, as previously discussed [Svaasand et al., 1996]. A high diffusivity $\left(\kappa=10^{-9} \mathrm{~m}^{2} / \mathrm{s}\right)$ and low clearance rate $(\tau=24 \mathrm{hrs})$ were used along with a stratum corneum permeability of $10^{-7} \mathrm{~m}^{2} / \mathrm{s}$ to $10^{-}$ ${ }^{8} \mathrm{~m}^{2} / \mathrm{s}$ for normal healthy tissue, and $10^{-6} \mathrm{~m}^{2} / \mathrm{s}$ for the diseased tissue. The steady state drug distributions were calculated for both types of tissue, and normalized to the surface concentration of the diseased tissue (Figure 22a). The theoretical model was then used to produce the steady state spatially resolved fluorescence curves (Figure 22b). These normalized curves have unique shapes and intensities, which illustrates how such measurements could provide information about the permeability of the stratum corneum.

The therapeutic ratio is the highest immediately after topical application (the tumorous region contains much more drug than the normal skin), and decreases with time until the steady state value is reached. To simulate the uptake of a photosensitizer by diseased tissue, drug distributions have been plotted at ten second intervals for the first minute following administration (Figure 23a). The diffusion model was then used to determine the spatially resolved fluorescence (using optical properties for $\mathrm{AlS}_{4} \mathrm{Pc}$ ), which was then normalized and plotted (Figure 23b). As the drug was taken up by the tissue, the fluorescence intensity decreased relative to the smallest radial distance. Each curve has a distinct slope and intensity, exhibiting the possibility of accurately monitoring the uptake at even small time intervals. 


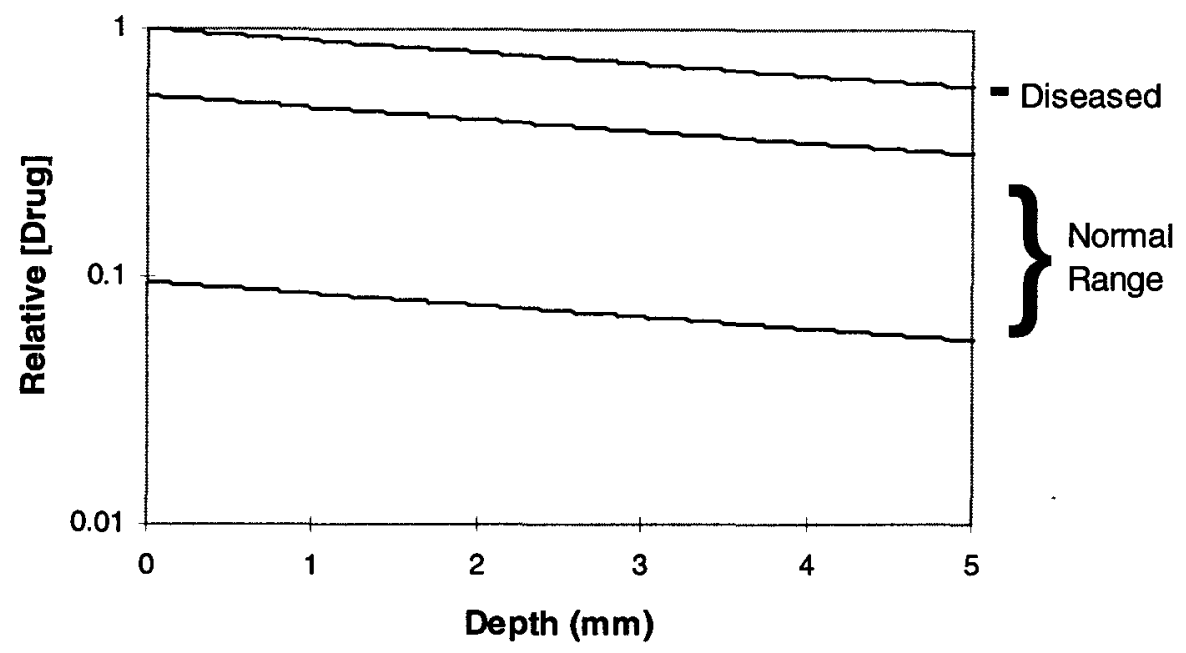

Figure 22a: Steady state drug concentrations for normal and diseased tissue, illustrating the increased permeability resulting from the breakdown of the stratum corneum.

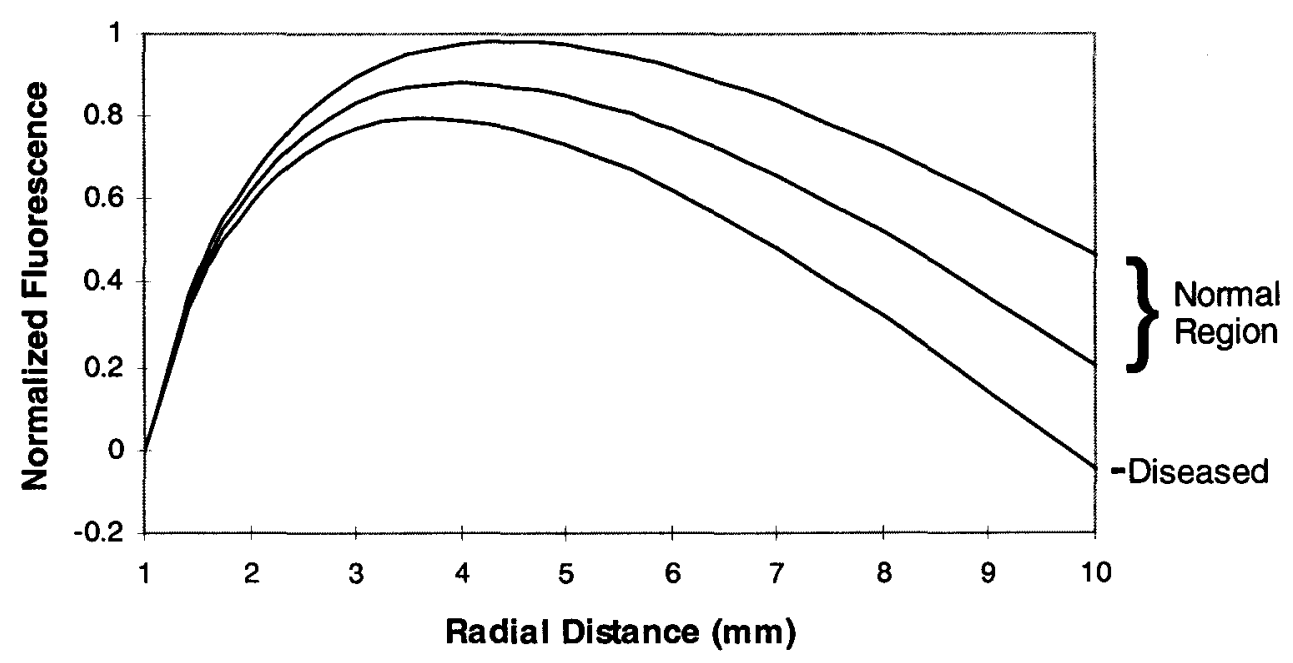

Figure 22b: Steady state spatially resolved fluorescence (natural logarithm of the fluorescence multiplied by the square of the radial distance, and normalized to a radial distance of $1 \mathrm{~mm}$ ). The optical properties for $\mathrm{AlS}_{4} \mathrm{Pc}$ were used along with the above drug distributions. 


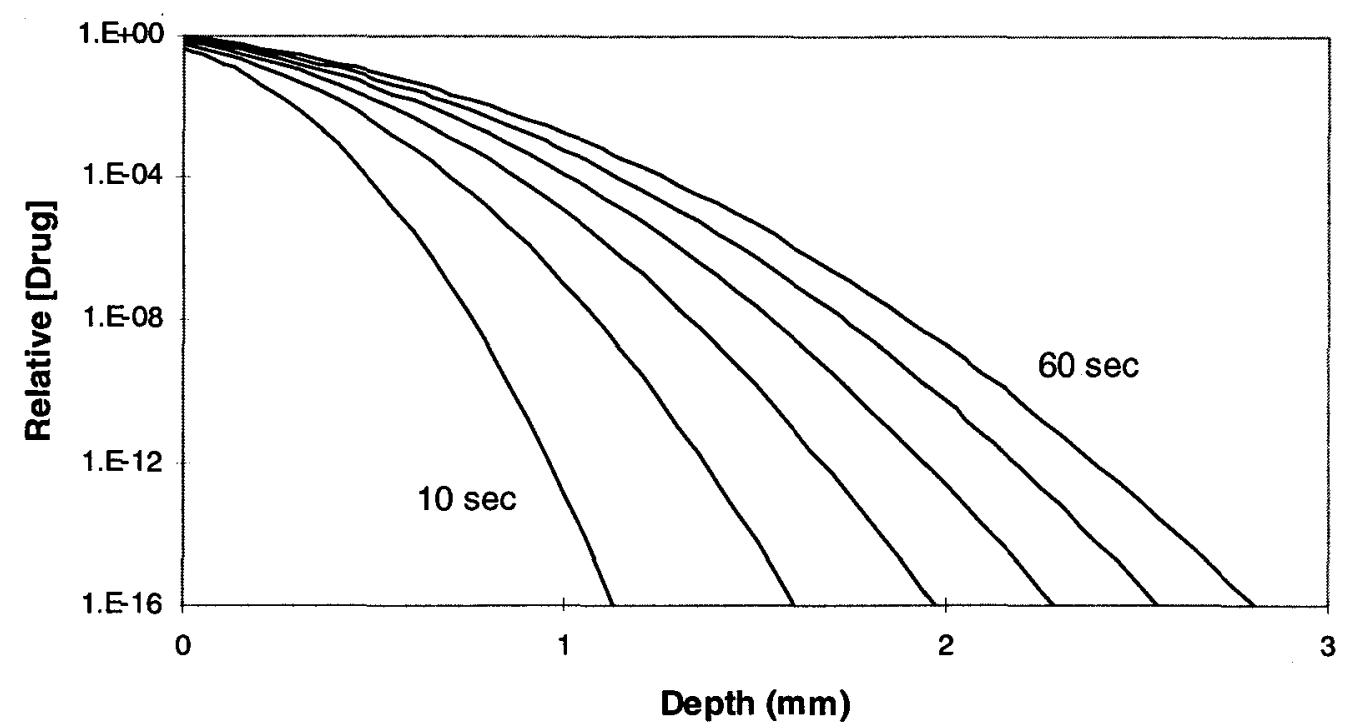

Figure 23a: Theoretical drug concentration at 10 second time intervals after the topical application of a photosensitizer (diseased tissue optical properties).

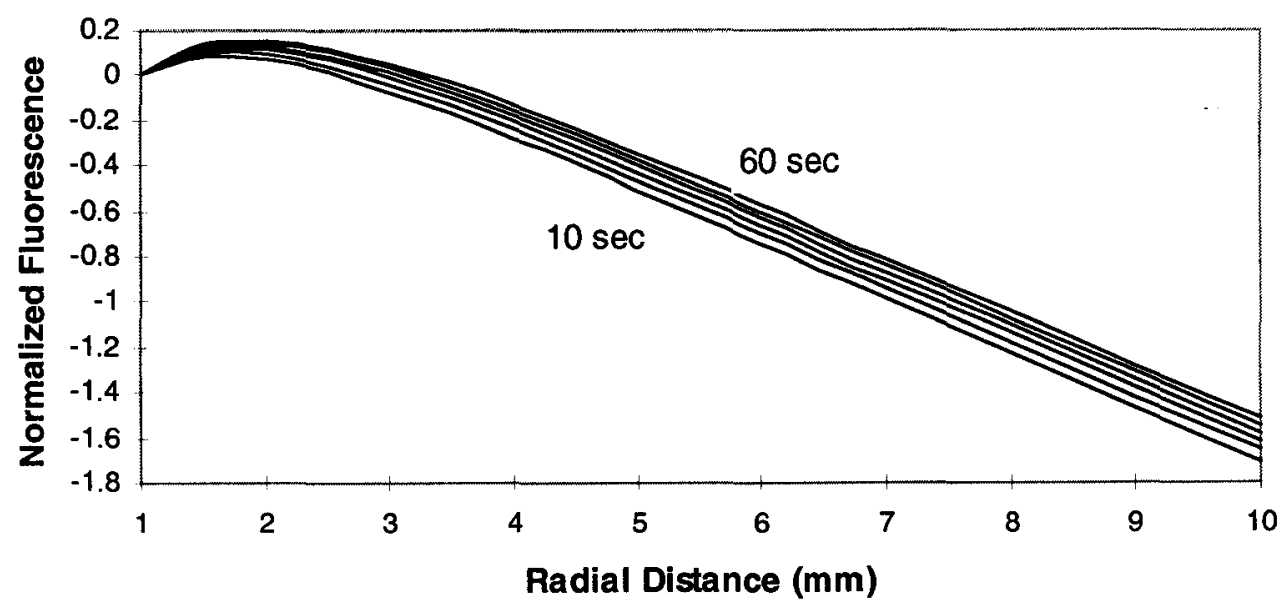

Figure 23b: Theoretical spatially resolved fluorescence at 10 second time intervals after the topical application of the photosensitizer (natural logarithm of the fluorescence multiplied by the square of the radial distance, and normalized to a radial distance of $1 \mathrm{~mm}$ ). The optical properties for $\mathrm{AlS}_{4} \mathrm{Pc}$ were used along with the above drug distributions. 


\subsection{Modeling Drug Bleaching during PDT}

The broad beam irradiation of a homogeneous drug concentration $(2 \mu \mathrm{g} / \mathrm{ml}$ $\mathrm{AlS}_{4} \mathrm{Pc}$ ) was considered first. The drug distribution was plotted at equal exposure intervals (fluence and time remain consistent), see Figure 24a, and the expected sigmoidal shape from the first order bleaching was observed. The spatially resolved fluorescence was then determined for the various distributions (Figure 24b). As the photosensitizer was bleached away from the surface, the fluorescence intensity increased relative to the smallest radial distance. The intensity and shape of each of these curves were also unique, illustrating the models potential to determine the depth of bleaching.

The bleaching of the drug distribution which was obtained 5 hours after topical administration to normal tissue was analysed. The distributions were plotted at equal exposure intervals (Figure 25a), and the spatially resolved fluorescence was calculated using optical properties of $\mathrm{AlS}_{4} \mathrm{Pc}$ (Figure 25b). The fluorescence was multiplied by the square of the radial distance, normalized to a radial distance of $1 \mathrm{~mm}$ (pre-treatment), and then the logarithm was plotted to accentuate the differences. The fluorescence intensity at the larger radial distances continually increases with bleaching (relative to the closest distance), which causes the slope of the curves to decrease. This indicates that the model could be a useful means of analysing the bleaching of a topically administered photosensitizer.

As the bleaching of the drug concentration was continued, some interesting boundary effects became apparent (Figure 25b). The concentration of the drug within 


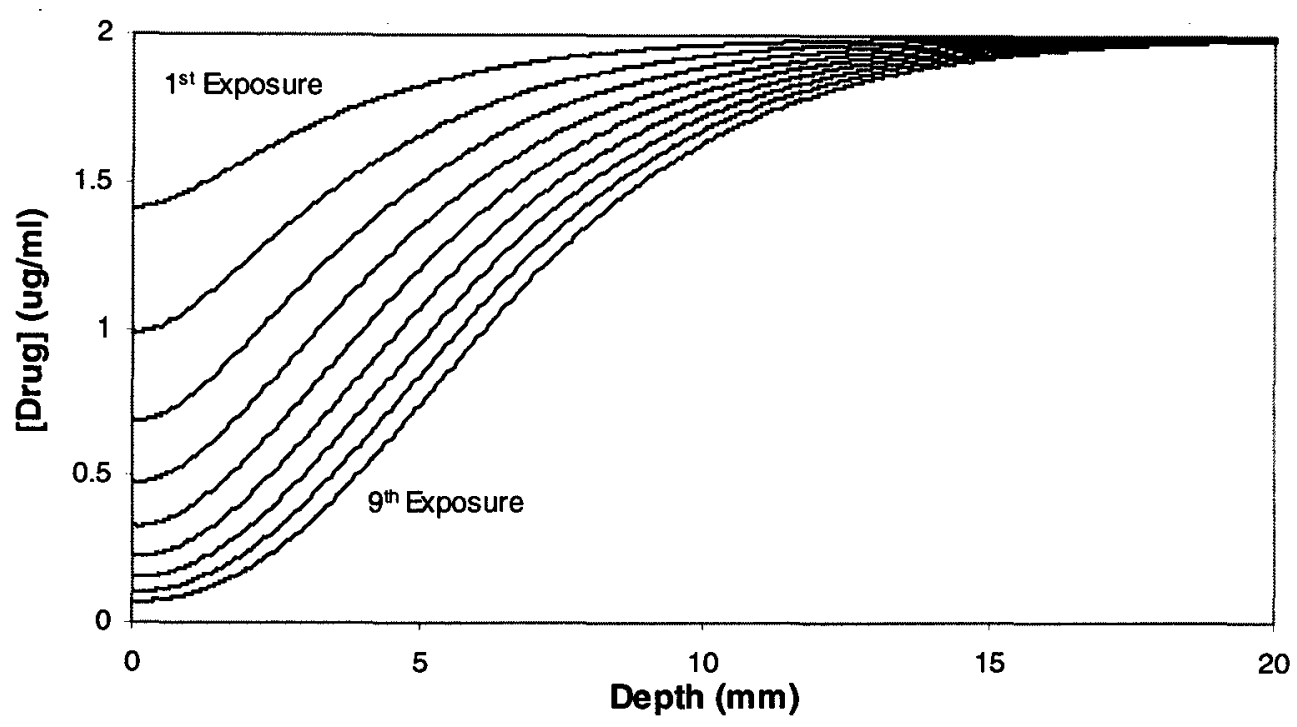

Figure 24a: Drug concentrations at consistent exposure intervals during the broad beam irradiation of a homogeneous drug concentration (optical properties for $2 \mu \mathrm{g} / \mathrm{ml} \mathrm{AlS}{ }_{4} \mathrm{Pc}$ ).

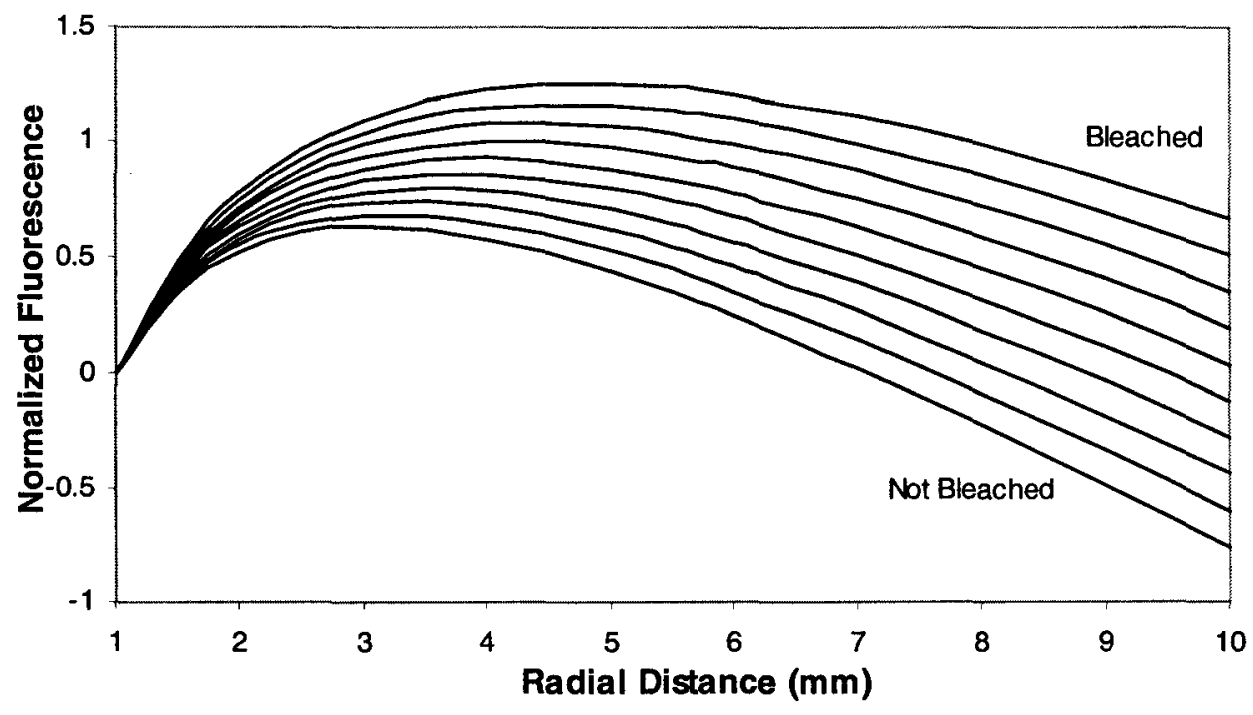

Figure 24b: Spatially resolved fluorescence at consistent exposure intervals during the broad beam irradiation of a homogeneous drug concentration (optical properties for 2 $\mu \mathrm{g} / \mathrm{ml} \mathrm{AlS}{ }_{4} \mathrm{Pc}$ ). Plotted as the natural logarithm of the fluorescence, multiplied by the square of the radial distance, and then normalized to a radial distance of $1 \mathrm{~mm}$ (to accentuate the differences). 


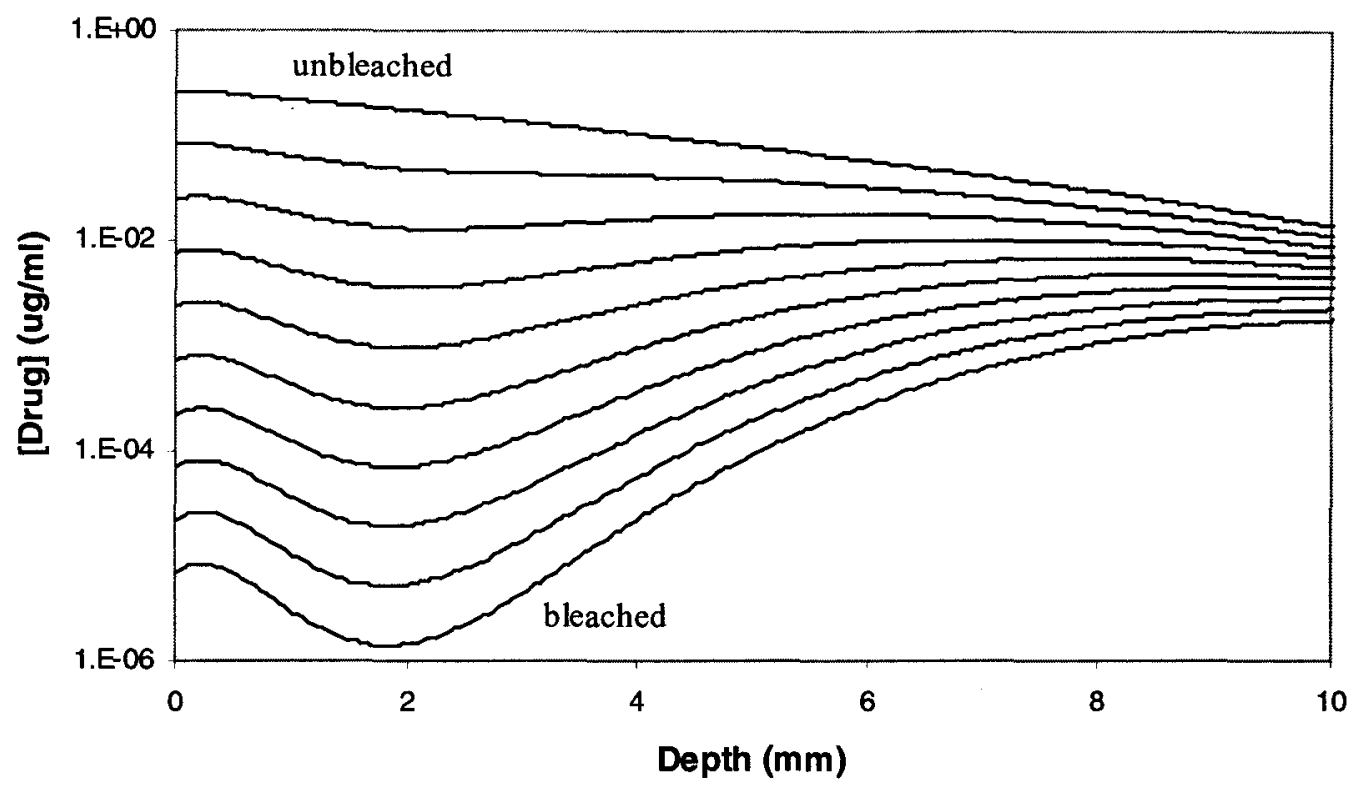

Figure 25a: Broad beam irradiation of the surface was performed $5 \mathrm{hrs}$ after the topical administration. Theoretical drug concentrations are shown after equal intervals of exposure.

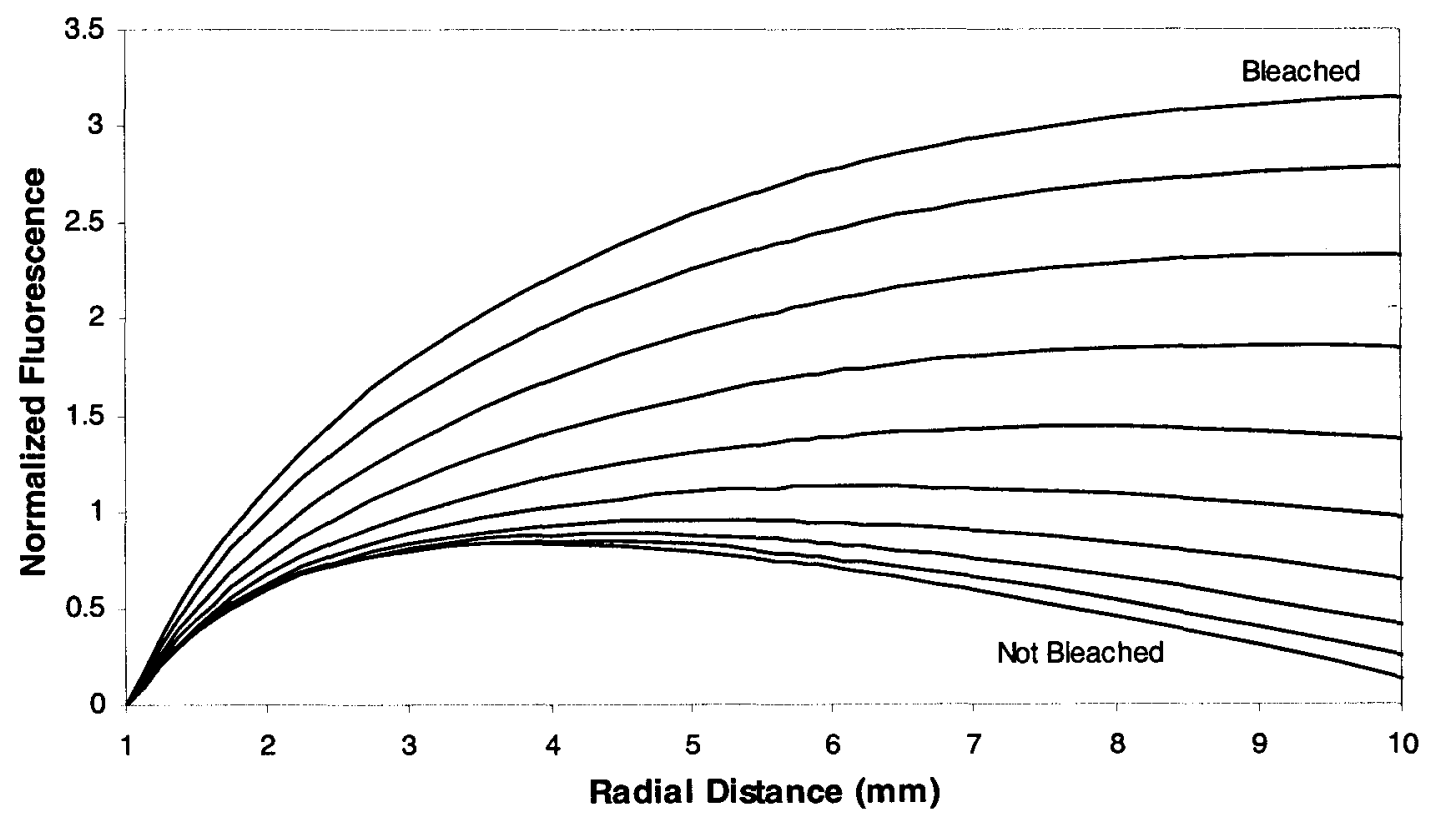

Figure 25b: Theoretical spatially resolved fluorescence (per photon) after equal intervals of exposure. The optical properties for $\mathrm{AlS}_{4} \mathrm{Pc}$ were used along with the above drug distributions. 
one mean free path from the surface $(\sim 1.5 \mathrm{~mm})$ remained slightly higher, due to the loss of the photons which were scattered from the medium (less backscatter). Superimposed on this phenomenon is a slight decrease in concentration at the surface, which was caused by the increased fluence from total internal reflection.

\subsection{Application to a Biological System}

As previously noted, this model could be a useful means of estimating a patient's drug concentration/distribution with a simple non-invasive measurement of the spatially resolved fluorescence. Likewise, the analysis of the reflectance data would provide additional information which may be useful in the determination of these properties. Although the physics may be readily modeled, the dynamic conditions in a biological environment are much more complex.

Using photosensitizer photobleaching as an index of the effective delivered dose would be a simple method of implicitly incorporating many of the dose parameters. Unfortunately the degree of oxygen coupling is not taken into account, and there are a number of complications with the optics and photosensitizers that must also be considered.

The measured fluorescence is averaged over a wavelength dependent tissue volume, which prevents the detection of the internal microscopic dynamics. If it were possible to use a shorter wavelength for the fluorescence excitation, the sampling volume would be reduced, allowing a more localized measurement of the photobleaching. Even if this is possible, it is not always practical. Thus measurements are often made at the 
treatment wavelength; without interruption. As a result, the tissue sampling volume is comparable to the PDT treatment volume. Unfortunately many photosensitizers have small Stokes shifts when activated by these long wavelengths, making the separation of the fluorescence emission signal difficult. It should also be noted that using photobleaching as an optical dosimetric is only relevant for photosensitizers that are significantly bleached at clinical light fluences [Wilson, 1997].

This model assumes that the absorption, fluorescence and photodynamic action spectra (as measured in solution) remain consistent for the duration of the irradiation procedure. This would not be a valid assumption if there was differential bleaching of separate photosensitizer constituents, or if the products of the bleaching were also photodynamically active. Furthermore, if the fluence rate of the excitation light is very high, measurements can be underestimated by the transient photobleaching caused by photosensitizer ground state depletion [Wilson, 1997].

If tissue autofluorescence constitutes a significant portion of the fluorescence, it must be dealt with in a reasonable manner. It should be noted that this may not be a trivial subtraction since it may decrease as a result of intrinsic fluorophore bleaching. Alternatively, a decrease in the autofluorescence could be caused by increased blood content during treatment, thereby enhancing the attenuation. Moreover, this model does not account for possible treatment induced changes in tissue optical properties [Wilson, 1997].

Finally, one must know the degree of oxygen coupling in the specific tissue environment to determine the dose. This may be affected by photosensitizer binding, 
photosensitizer structure, tissue type, or even the injection to irradiation time. If one is to assess the biological damage, it is also important to note that the oxygen concentration may be much higher in lipid membranes than cytosol [Wilson, 1997]. 


\section{CONCLUSION}

It should be possible to use spatially resolved fluorescence measurements to monitor the uptake of a topically applied photosensitizer, as well as its bleaching during PDT. The previous simplified model involved assumptions which were not valid at clinical wavelengths. The fundamental basis of this new model is appropriate, and its accuracy has been demonstrated for simple geometries by comparison to Monte Carlo simulations and phantoms. Its computation time is also quite reasonable.

This model has shown that spatially resolved fluorescence may be a useful means of optimizing the time between topical administration and irradiation, as well as the duration of the treatment itself. Intermittent measurements could also allow 'real-time' comparison of the treatment to what was actually prescribed. Finally, the quantification of the photobleaching may allow an implicit determination of the dose. Unfortunately, the introduction to a biological system generates many other factors which must also be taken into account. Once singlet oxygen production and biological damage are extensively modeled, it should be possible to determine a clinical dose. This would certainly improve the biological effectiveness of treatments which utilize topically administered photosensitizers.

Future endeavors will undoubtedly involve the verification of the model for varying drug concentrations in phantoms, such as those produced by photobleaching. A 
number of in-vivo experiments could be performed to assess the ability to monitor diffusion as well as the final concentration within a layer of tissue (such as a layer of skin). The model could then be used to fit experimental data and extract useful parameters. Ultimately, it could be used in conjunction with other information to measure biological effectiveness. 


\section{APPENDIX 1: PHANTOM MATERIALS}

\section{Liquid:}

A solution consisting of $200 \mathrm{ml} \mathrm{H} \mathrm{H}_{2} \mathrm{O} 5 \mathrm{ml}$ of $20 \%$ intralipid $^{\circledR}$ solution (or Travamulsion $^{\mathbb{B}}$ ), and $1 \mu$ India ink produces optical properties of approximately $\mu_{\mathrm{s}}{ }^{\prime}=1$ $\mathrm{mm}^{-1}$ and $\mu_{\mathrm{a}}=0.01 \mathrm{~mm}^{-1}$. It allows excellent probe coupling, and the ability to readily produce phantoms with a variety of different drugs and drug concentrations for comparison. Plastic 'sandwich' wrap was used to separate liquid layers for a two-layer phantom, and as previously noted, any boundary affect was found to be insignificant. The experimental results, however, occasionally suggested a reduction in the amount of photo-active drug. It is thought that this was caused by aggregation and binding with the Intralipid $^{\circledR}$ suspension. As previously noted, a liquid phantom isn't practical for the analysis of photobleaching since it allows the mixing of its various constituents.

\section{Polystyrene Resin:}

Polystyrene phantoms were constructed by dissolving $0.95 \mathrm{~g}$ of $\mathrm{TiO}_{2}$ in $5 \mathrm{ml}$ of methanol. This was then added to $200 \mathrm{ml}$ of Lewiscraft ${ }^{\circledR}$ clear casting resin, and the $\mathrm{TiO}_{2}$ was rinsed from the beaker with an additional $5 \mathrm{ml}$ of methanol until little remained. To this, $1 \mu \mathrm{l}$ of India ink and any desired photosensitizer was added, and the constituents 
were stirred to form a homogeneous mixture. The phantom started setting with the addition of 12-15 drops of catalyst. The heat produced from this polymerization may raise the temperature of the medium from room temperature to over $40^{\circ} \mathrm{C}$. Consequently, the effect of this temperature increase on the photosensitizer must be considered since it is generally only used at body temperature. After approximately 24 hours, the phantom is slightly tacky, but after 48 hours it is fully cured. The top of the phantom is sanded smooth to remove the glossy finish and provide a flat surface for improved probe coupling. A coupling agent such as water or gel is still beneficial. Unfortunately, the $\mathrm{TiO}_{2}$ is slightly denser than the resin and some settling occurs while it cures. Regardless, this produces a very solid phantom with reproducible optical properties which are approximately $\mu_{\mathrm{s}}{ }^{\prime}=1 \mathrm{~mm}^{-1}$ and $\mu_{\mathrm{a}}=0.01 \mathrm{~mm}^{-1}$ when measured at the surface.

\section{Silicone:}

Four microlitres $(4 \mu 1)$ of dye and any desired photosensitizer were added to 200 $\mathrm{ml}$ of Silicone (Rhodorsil ${ }^{\circledR}$ RTV-141). As the hardener is much less viscous than the silicone, $10 \mathrm{ml}$ of hardener was used to dissolve $0.7 \mathrm{~g}$ of $\mathrm{TiO}_{2}$. This was then added to the solution. Since the solution is not very dense, the mixture must be put in a vacuumed container to remove any air bubbles from the medium. Once the bubbles no longer appear at the surface, the mixture is left to cure for 24 hours. Again, settling of the $\mathrm{TiO}_{2}$ is a small problem. This procedure produces a solid, though compressible, phantom with 
excellent probe coupling. Again, this produces optical properties which are approximately $\mu_{\mathrm{s}}^{\prime}=1 \mathrm{~mm}^{-1}$ and $\mu_{\mathrm{a}}=0.01 \mathrm{~mm}^{-1}$.

Agar:

Two grams $\left(2 \mathrm{~g}\right.$ ) of purified agar (from Sigma ${ }^{\circledR}$ ) is added to a beaker with $200 \mathrm{ml}$ of $\mathrm{H}_{2} \mathrm{O}$, which is placed on a hotplate with a magnetic stirrer. The solution is heated to $98^{\circ} \mathrm{C}$, and then placed in an ice-water bath. While continuously stirring, it is cooled to $40^{\circ} \mathrm{C}$ and then $9 \mathrm{ml}$ of $20 \%$ Intralipid $^{\circledR}, 9 \mu 1$ of Higgin's ${ }^{\circledR}$ dye and any desired photosensitizer are added. These constituents are continuously stirred into solution until the temperature drops to $30^{\circ} \mathrm{C}$. At this time, the mixture is poured into an appropriate container and left to set at room temperature. It begins to solidify quite rapidly so settling is not a concern. After 24 hours the phantom is fully set. This forms a gelatin-like solid which can easily be torn by the probe when making measurements. Once the phantom is set, it must be covered to prevent dehydration, which can dramatically change both its structure and optical properties. This procedure approximately yields optical properties with $\mu_{\mathrm{s}}{ }^{\prime}=1.5 \mathrm{~mm}^{-1}$ and $\mu_{\mathrm{a}}=0.04 \mathrm{~mm}^{-1}$. 


\section{APPENDIX 2: FLUOROPHORES}

\section{Aluminum Pthalocyanine (tetra-sulphonate)}

This drug is extensively used experimentally, and has been used in our layered experiments because it is excited by a HeNe laser, it has a good fluorescence yield, and its bleaching is not significant. Unfortunately the absorption at its fluorescence wavelength $\left(\sim 0.025 \mathrm{~mm}^{-1}(\mu \mathrm{g} / \mathrm{ml})^{-1}\right.$ at $\left.683 \mathrm{~nm}\right)$ is an order of magnitude stronger than the absorption at the excitation wavelength $\left(\sim 0.00599 \mathrm{~mm}^{-1}(\mu \mathrm{g} / \mathrm{ml})^{-1}\right.$ at $\left.632 \mathrm{~nm}\right)$, so counting statistics suffer. Regardless, it has provided excellent experimental results which are comparable to theory. It should also be noted that a Royal Lavender filter is useful in resolving the fluorescence from the excitation.

\section{Photofrin $^{\circledR}$}

This drug is also used extensively in this field, and its photobleaching is not very significant. It has a strong absorption at $632 \mathrm{~nm}$ but its fluorescence spectrum is quite complex, with various emission wavelengths. Consequently, it is less than ideal from a modeling standpoint. 


\section{DCM}

This is a red powder obtained from Exciton Inc., and it is the dye which is used in the argon ion-pumped dye laser. It has a solubility of $280 \mu \mathrm{g} / \mathrm{ml}$ in Methanol, and is also soluble in water. It has an absorption coefficient of $0.0200 \mathrm{~mm}^{-1}(\mu \mathrm{g} / \mathrm{ml})^{-1}$ at $488 \mathrm{~nm}$ and $0.0117 \mathrm{~mm}^{-1}(\mu \mathrm{g} / \mathrm{ml})^{-1}$ at $515 \mathrm{~nm}$. It emits fluorescence after the absorption of both these wavelengths. When it is contained in a polystyrene resin phantom, it only bleaches with a high intensity $488 \mathrm{~nm}$ excitation (observed using a pencil beam geometry). Consequently, it would be useful to use a $488 \mathrm{~nm}$ broad beam irradiation to bleach a phantom, while intermittently using a $515 \mathrm{~nm}$ excitation to acquire the spatially resolved fluorescence, without subsequently bleaching that volume. Fortunately the absorption of this compound decreases quite rapidly after $515 \mathrm{~nm}$, so fluorescence re-absorption is not a significant problem. The Canary filter provides separation of the excitation from the fluorescence.

\section{Fluorescein Isothiocyanate-Inulin}

This drug has previously been used for in vivo rabbit experiments in our laboratory. Photobleaching is evident in these experiments, but it is complicated by other biological processes. Consequently, it would be useful to study this drug in a phantom so that the drug concentration and optical properties can be controlled. It is excited at 488 $\mathrm{nm}$, and when its contained in polystyrene resin it fluoresces and bleaches as well. Again, the Canary filter provides excellent detection of the fluorescence. 


\section{Ethidium Bromide}

This is a strongly mutagenic compound which is used in the biology laboratories to label DNA. It absorbs at $515 \mathrm{~nm}$ and emits fluorescence at $595 \mathrm{~nm}$, which isn't significantly re-absorbed. When contained in a polystyrene resin phantom it fluoresces and bleaches, but isn't quite as safe to work with.

\section{Flazo Orange}

This is a powder from Sigma which absorbs at 488 and $515 \mathrm{~nm}$, and emits fluorescent light at $612 \mathrm{~nm}$. It strongly fluoresces in methanol, but unfortunately it doesn't at all in water.

\section{Rhodamine 123}

This is a relatively expensive drug which is also excited at the argon wavelengths, and emits fluorescence as $534 \mathrm{~nm}$. The absorption coefficients were determined to be

$$
\begin{aligned}
& 0.0239 \mathrm{~mm}^{-1}(\mu \mathrm{g} / \mathrm{ml})^{-1} \text { at } 488 \mathrm{~nm}, \\
& 0.0572 \mathrm{~mm}^{-1}(\mu \mathrm{g} / \mathrm{ml})^{-1} \text { at } 515 \mathrm{~nm}, \\
& 0.0076 \mathrm{~mm}^{-1}(\mu \mathrm{g} / \mathrm{ml})^{-1} \text { at } 534 \mathrm{~nm} .
\end{aligned}
$$




\section{REFERENCES}

Abramowitz M. and I.A. Stegun, 1972. Handbook of Mathematical Functions..., Dover Publications, Inc., New York. p371, 409, 916.

Alexandrakis, G., T.J. Farrell and M.S. Patterson, 1998. Accuracy of the diffusion approximation in determining the optical properties of a two-layer turbid medium. App. Opt. 37 7401-7409.

Bruulsema, J. 1997. Design and Testing of a Noninvasive Steady-stage Device for the Measurement of Optical Properties of Tissues in the 900-1400 nm Wavelength Region. Master's Thesis, McMaster University.

Duderstadt J.J. and L. J. Hamilton, 1976. Nuclear Reactor Analysis. Wiley, New York.

Farrell, T.J., R.P. Hawkes, M.S. Patterson and B.C. Wilson, 1998. Modeling of photosensitizer fluorescence emission and photobleaching for photodynamic therapy dosimetry. App Opt 37 7168-7183.

Fuchs, J. and J. Thiele, 1998. Review Article - The Role of Oxygen in Cutaneous Photodynamic Therapy. Free Radical Bio \& Med. 5 835-847.

Gerald, C.F., P.O. Wheatly, Applied Numerical Analysis $5^{\text {th }}$ edition, 1994. AddisonWesley Publishing Co. Inc. 473-482, 549-552, 620, 666-668.

Hasegawa, Yasuo et al, 1991. Monte Carlo simulation of light transmission through living tissues. App Opt 31 4515-4520.

Haskell, R.C., L.O. Svaasand, T.T.Tsay, T.C. Feng, MN. McAdams and B.J. Tromberg, 1994. Boundary conditions for the diffusion equation in radiative transfer. J Opt Soc Am A $112727-2741$.

Hawkes, R.P. 1997. Photodynamic Therapy Dosimetry Through Measurement of Fluorescence Decrease Due to Photobleaching. Master's Thesis, McMaster University.

Henyey L.G. and Greenstein J.L., 1941. Diffuse radiation in galaxy. Astrophys. 93 70-83. 
Hulst, H.C. van de, 1980. Multiple Light Scattering Tables, Formulas and Applications. Academic Press, New York.

Kienle, A., M.S. Patterson, N. Utke, R. Bays, G. Wagnieres, and H. Van Den Bergh, 1998. Determination of the optical properties of a two-layer turbid media. App. Opt. 37 779-791.

Kienle, A. and M.S. Patterson, 1997. Improved solutions of the steady-state and timeresolved diffusion equations for reflectance from a semi-infinite turbid medium. J Opt Soc Am A 14 246-254.

Kienle, Alwin and Michael S. Patterson, 1996. Determination of the optical properties of turbid media from a single Monte Carlo simulation. Phys Med Bio 41 2221-2227.

McCaughan, J.S. 1992. A Clinical Manual: Photodynamic Therapy of Malignancies, R.G. Landes Company, Austin.

Nossal, R, J. Kiefer, G.H. Weiss, R. Bonner, H. Taitelbaum and S. Havlin, 1988. Photon migration in layered media. App Opt 27 3382-3391.

Patterson, M.S. 1995. Noninvasive Measurements of Tissue Optical Properties: Current Status and Future Prospects, Mol. Cell. Biophys. 6 387-417.

Patterson, M.S. and B.W. Pogue, 1994. Mathematical model for time-resolved and frequency-domain fluorescence spectroscopy in biological tissues, App Opt. 10 19631974.

Potter, W.R. 1986. The theory of photodynamic therapy dosimetry: Consequences of photodestruction of sensitizer. International Soc. for Opt. Eng. 712 124-129.

Press, W.H. et al., 1996. Numerical Recipes in Fortran 77: The Art of Scientific Computing, Cambridge University Press, U.S.A..

Profio, A.E. 1979. Radiation Shielding and Dosimetry, Wiley, New York. 168-199.

Reichman, J. 1973. Determination of Absorption and Scattering Coefficients for Nonhomogeneous Media, App Opt 8 1811-1815.

Rider, D. 1999. Personal communication with Reynolds ${ }^{\circledR}$ (producers of Reynolds wrap).

Svaasand, L.O. and B. Tromberg, 1991. Future Trends in Biomedical Applications of Lasers, Proc. Soc. Photo-Opt. Instrum. Eng. 1525 41-51. 
Svaasand, L.O., P. Wyss, M. Wyss, Y Tadir, B.J. Tromberg and M.W. Berns, 1996. Dosimetry Model for Photodynamic Therapy with topically administered photosensitizers. Lasers Surg Med 18 139-149.

Weersink, R.A., J.E. Hayward, K.R. Diamond and M.S. Patterson, 1997. Accuracy of noninvasive in vivo measurements of photosensitizer uptake based on a diffusion model of reflectance spectroscopy. Photochem Photobiol 66 326-335.

Wilson, B.C., M.S. Patterson, L. Lilge, 1997. Review Article, Implicit and Explicit Dosimetry in Photodynamic Therapy: a New Paradigm. Lasers in Med Sci 12 182-199.

Wilson, B.C. and G. Adam, 1983. A Monte Carlo model for the absorption and flux distribution of light in tissue. Med Phys. 10 824-830.

Yoo, K.M., Feng Liu, and R.R. Alfano, 1990. When does the Diffusion Approximation Fail to Describe Photon Transport in Random Media, Phys Rev Let 22 2647-2650. 